\title{
Otimização em Meteorologia: cálculo de perturbações condicionais não-lineares ótimas
}

\author{
Jessé Américo Gomes de Lima
}

\author{
DisSERTAÇÃO A SER APRESENTADA \\ $\mathrm{AO}$ \\ Instituto DE MATEMÁticA E EstatísticA \\ DA \\ Universidade DE SÃo PaUlo \\ PARA \\ OBTENÇÃO DO TÍTULO \\ DE \\ Mestre EM CiÊnCIAS
}

Programa: Mestrado em Ciência da Computação Orientador: Prof. Dr. Ernesto Julián Goldberg Birgin

Durante o desenvolvimento deste trabalho o autor recebeu auxílio financeiro do CNPq.

São Paulo, maio de 2012. 


\section{Otimização em Meteorologia: cálculo de perturbações condicionais não-lineares ótimas}

Esta versão da dissertação contém as correções e alterações sugeridas pela Comissão Julgadora durante a defesa da versão original do trabalho, realizada em 11/05/2012. Uma cópia da versão original está disponível no Instituto de Matemática e Estatística da Universidade de São Paulo.

Comissão Julgadora:

- Prof. Dr. Ernesto Julián Goldberg Birgin - IME-USP

- Prof. Dr. Júlio Michael Stern - IME-USP

- Prof. Dr. Ricardo Caetano Azevedo Biloti - IMECC-UNICAMP 


\section{Resumo}

LIMA, J. A. G. Otimização em Meteorologia: cálculo de perturbações condicionais nãolineares ótimas. 2012. 43 f. Dissertação (Mestrado) - Instituto de Matemática e Estatística, Universidade de São Paulo, São Paulo, 2012.

Neste trabalho estudamos as aplicações do método do Gradiente Espectral Projetado (SPG) em Meteorologia nos campos de previsibilidade, estabilidade e sensibilidade. Inicialmente revisamos os Vetores Singulares Lineares (LSVs) e em seguida apresentamos a teoria das Perturbações Condicionais Não-Lineares Ótimas (CNOPs). Enquanto os métodos clássicos estão baseados no Modelo Tangente Linear, as CNOPs são uma formulação do mesmo problema baseado em Programação Não-Linear. As CNOPs são descritas na literatura como responsáveis por melhorias em relação aos métodos anteriores. Finalmente analisamos três exemplos de aplicação do método à problemas de previsibilidade, estabilidade e sensibilidade.

Palavras-chave: Meteorologia, SPG, previsibilidade, estabilidade, sensibilidade, CNOPs, modelo tangente linear. 


\section{Abstract}

LIMA, J. A. G. Optimization in Meteorology: computation of conditional nonlinear optimal perturbations. 2012. 43 f. Dissertação (Mestrado) - Instituto de Matemática e Estatística, Universidade de São Paulo, São Paulo, 2012.

A revision about applications of Spectral Projected Gradient (SPG) in meteorology is done in the fields of predictability, stability and sensitivity. Initially we review about Linear Singular Vectos (LSVs) and we present the Conditional Nonlinear Optimal Perturbations (CNOPs). While the classic methods are based on the Tangent Linear Model, CNOPs are another formulation of the problem based on Nonlinear Programming. CNOPs are described in bibliography as responsible by better results than older methods. Finally we analyze three applications in predictability, stability and sensibility.

Keywords: meteorology, SPG, predictability, stability, sensibility, CNOP, tangent linear model. 


\section{Sumário}

1 Introdução 1

2 Conceitos básicos $\quad 3$

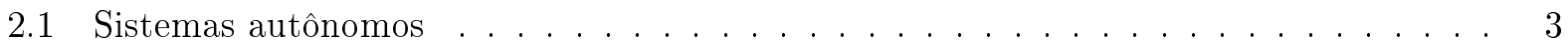

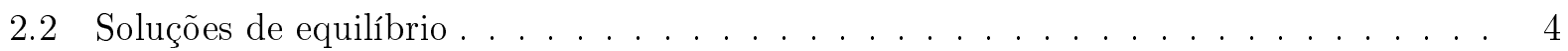

2.3 Estabilidade de uma solução de equilíbrio . . . . . . . . . . . . . . . . . . 4

2.4 Linearização ao redor de uma solução de equilíbrio . . . . . . . . . . . . . . . . 4

3 Perturbações condicionais não-lineares ótimas $\quad 7$

3.1 Propagador e modelo tangente linear . . . . . . . . . . . . . . . 7

3.2 Valores e vetores singulares lineares . . . . . . . . . . . . . . . 8

3.3 Perturbações condicionais não-lineares ótimas . . . . . . . . . . . . . . . . 9

3.4 Método de Runge-Kutta . . . . . . . . . . . . . . . . . . . . . . . . . 9 9

3.5 Método do gradiente espectral projetado . . . . . . . . . . . . . . . . . . 10

3.5 .1 Algoritmo SPG . . . . . . . . . . . . . . . . . . 10

4 Testes preliminares $\quad 12$

4.1 Modelo de Lorenz de $1963 \ldots \ldots$. . . . . . . . . . . . . . . . . 12

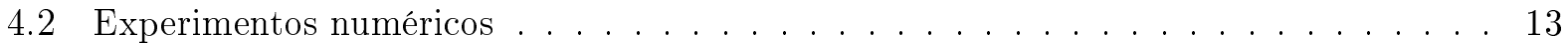

5 Aplicações em previsibilidade, estabilidade e sensibilidade $\quad 21$

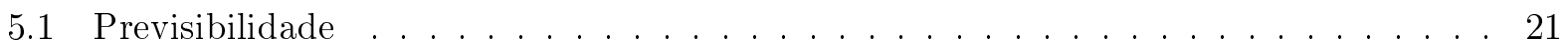

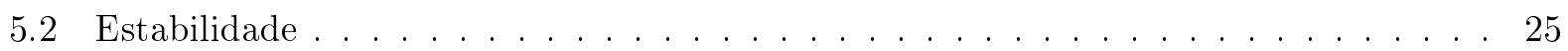

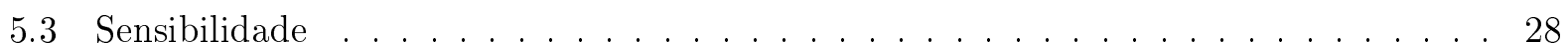

6 Conclusões 35

Apêndice A Código fonte dos modelos $\quad 36$

A.1 O Modelo de Lorenz de $1963 \ldots \ldots$. . . . . . . . . . . . . . . 36

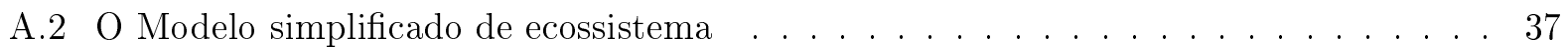

A.3 O Modelo de Lorenz de $1996 \ldots \ldots$. . . . . . . . . . . . . . . . 40

$\begin{array}{ll}\text { Referências Bibliográficas } & 41\end{array}$ 


\section{Capítulo 1}

\section{Introdução}

O método do Gradiente Espectral Projetado (SPG) [1, 2] é um método utilizado para minimizar funções contínuas e diferenciáveis em conjuntos convexos. O método foi introduzido em 2000 e, desde então, utilizado em diversas aplicações práticas. Neste trabalho estamos interessados em estudar as aplicações do SPG a problemas de otimização em Meteorologia $[3,4,5,6,7,8,9,10,11,12$, 13]. Dentro desta grande área, o SPG é utilizado para analisar a previsibilidade, estabilidade e sensibilidade [3] de modelos matemáticos utilizados na previsão numérica de tempo e clima. Em particular, a maioria das aplicações está relacionada com a resolução de subproblemas de otimização que surgem na aplicação de um método chamado perturbação condicional não-linear ótima (CNOP, do inglês Conditional Nonlinear Optimal Perturbation) [14]. O conteúdo do texto a seguir está fortemente baseado no trabalho de Duan [15].

Previsão numérica de tempo e clima consiste basicamente em resolver um sistema de equações diferenciais parciais não-lineares. Este sistema descreve a evolução dos fenômenos atmosféricos baseados nas leis físicas que os regem, a partir de uma condição inicial (veja, por exemplo, [16]). Tais sistemas são referidos como "modelos" e inicialmente formulados em sua "forma diferencial". Estes sistemas são então discretizados no espaço e no tempo por métodos numéricos adequados e a solução do modelo é obtida através de sua integração numérica. Este processo é comumente chamado de "rodar o modelo" e o resultado da operação nos dá a solução "não-linear" do mesmo, que expressa o resultado da evolução do fenômeno modelado durante o tempo de integração.

Entretanto existem grandes dificuldades inerentes ao estudo de tais modelos. Estas dificuldades são impostas pela complexidade, não-linearidade e multiplicidade dos fatores atuantes na evolução dos fenômenos. Além disso, é impossível incorporar de maneira precisa todos os fatores atuantes na atmosfera através dos processos de modelagem numérica. Isto porque, se por um lado, a teoria sobre a qual se baseiam esses modelos possui deficiências na descrição física dos fenômenos, por outro, incorporar todos os processos em alto nível de detalhamento tornaria a previsão numérica de tempo proibitiva dado o custo computacional que se assumiria. Por estes fatores, a simulação numérica de tempo é um tópico recorrente no campo de atuação da computação científica desde o advento do primeiro computador digital de grande porte nos anos 50 .

Dado que os processos de modelagem não captam o fenômeno de maneira ideal, faz-se necessário que se estime a incerteza ou o erro associado a uma dada previsão. O ato de estimar tais incertezas e o estudo da sua evolução no tempo são conhecidos como problemas de "previsibilidade". Lorenz [16] foi pioneiro nos estudos desta área, sendo o primeiro a propor o conceito do modelo tangente linear (TLM, do inglês Tangent Linear Model) na abordagem da questão da previsibilidade de um modelo. O TLM consiste numa aproximação linear de um sistema não-linear. Assim, ao descartar os termos de ordem superior da expansão em série do sistema original, obtém-se uma descrição aproximada do mesmo. A solução a partir da condição inicial é obtida através de integração utilizando os mesmos métodos numéricos empregados na solução do modelo não-linear.

Os estudos de Lorenz [16] introduziram e nortearam todo o tratamento posterior dado ao tema. A abordagem em [16], que utiliza vetores singulares lineares (LSVs, do inglês Linear Singular Vectors) 
e é baseada no TLM, tem sido largamente utilizada na Meteorologia Operacional. Os LSVs representam a medida do crescimento linear de perturbações iniciais e têm sido utilizados na estimativa do crescimento de erros em previsões e na análise de sensibilidade e estabilidade de sistemas. Embora o TLM tenha se tornado a abordagem clássica do assunto, a premissa de que a evolução de um sistema não-linear possa ser descrita por uma aproximação linear é um procedimento que levanta dúvidas a respeito de sua adequação. Nos problemas de previsibilidade, verificou-se que LSVs não representam bem o papel das incertezas nas condições iniciais com o maior efeito nos resultados da previsão. Segundo Duan [3] uma série de estudos já discutiram esse tema e são concordantes na ideia de que determinar a validade do TLM como aproximação para o modelo não-linear é uma tarefa complexa e essencialmente empírica.

Assim, estudando os efeitos da não-linearidade na amplificação de perturbações nas condições iniciais em modelos, Mu e Duan [17, 18] propuseram os conceitos de vetores singulares não-lineares e valores singulares não-lineares (NSVs e NSVAs, do inglês Nonlinear Singular Vectors e Nonlinear Singular Values), utilizando diretamente os modelos não-lineares. No entanto, o método apresentava limitações de ordem prática que o tornava desinteressante em aplicações. Posteriormente, no trabalho de $\mathrm{Mu}$ et al. [14], foi enunciada a teoria das CNOPs que tem se mostrado superior em captar a não-linearidade intrínseca aos fenômenos atmosféricos e oceânicos quando comparada com os LSVs. Enquanto os LSVs aproximam o crescimento de perturbações iniciais linearmente, CNOPs consistem na medida da evolução não-linear de tais perturbações numa previsão.

Desde que foram propostas, CNOPs foram utilizadas em estudos sobre a previsibilidade de modelos, além de aplicadas a problemas de estabilidade e sensibilidade $[3,4,5,6,7,8,9,10,11,12$, 13]. A referência [11], cita o problema da Oscilação Sul do El-Niño, (ENSO, do inglês El-Niño Southern Oscillation) como objeto de vários estudos demonstrando que procedimentos envolvendo CNOPs na inicialização de modelos podem melhorar de maneira significativa a previsibilidade de alguns fenômenos. Em [3] é citada a utilização das CNOPs nos estudos do crescimento dos erros iniciais do ENSO no modelo teórico de Zebiak-Cane, modelo este extensivamente utilizado na investigação do erro inicial que causa a maior incerteza na previsão do ENSO. Em [5], aborda-se a questão da previsão de tempo pelo método ensemble, tornando a previsão numérica de tempo num problema estocástico ao invés de determinístico, configurado como uma estimativa de probabilidades das condições de tempo futuras. Já em [8], segundo os autores, a evolução do crescimento de erros do tipo CNOP numa previsão do El-Nino demonstra uma proeminente dependência com relação ao período do ano, e a relaciona às CNOPs como a incerteza mais impactante dos resultados.

Em todos os casos enumerados anteriormente observa-se de imediato que o método do Gradiente Espectral Projetado (SPG) tem sido citado como uma alternativa viável e eficiente na solução dos problemas associados à determinação das CNOPs em toda a gama das aplicações do método [15, 19]. Nossa motivação, portanto, é estudar de que forma o SPG é utilizado para resolver os problemas de previsibilidade, sensibilidade e estabilidade na área da Meteorologia.

O restante deste trabalho está organizado como se segue. No Capítulo 2 revisamos alguns conceitos básicos necessários à descrição de sistemas de equações diferenciais ordinárias e análise de estabilidade. No Capítulo 3 estudamos os conceitos de modelo tangente linear, vetores singulares lineares, perturbações condicionais ótimas não-lineares e a família de métodos de Runge-Kutta. Também revemos o método do gradiente espectral projetado e o algorítimo do SPG. No Capítulo 4 apresentamos os experimentos numéricos que realizamos no intuito de avaliar nossa compreensão do tema e validar nosso código de obtenção dos LSVs e CNOPs. No Capítulo 5 reproduzimos três estudos de caso nos tópicos de previsibilidade, estabilidade e sensibilidade utilizando CNOPs. No Capítulo 6 tecemos comentários finais a respeito dos resultados deste trabalho. 


\section{Capítulo 2}

\section{Conceitos básicos}

Uma equação diferencial é uma equação com uma função desconhecida que depende de uma ou várias variáveis e que relaciona os valores da função e suas derivadas. Equações diferenciais cumprem um papel importante na modelagem de diversos processos em Física, Biologia e Engenharia, que vão desde movimento de corpos celestes até desenho de pontes.

Revisamos a seguir alguns conceitos básicos de equações diferenciais ordinárias de primeira ordem necessários para descrever formalmente o problema de otimização resolvido pelos meteorologistas usando o SPG. Estes conceitos básicos incluem as soluções de equilíbrio de sistemas, a estabilidade de uma solução de equilíbrio e análise linear da estabilidade. O conteúdo do texto a seguir está fortemente baseado nas referências [20, 21].

\section{$2.1 \quad$ Sistemas autônomos}

Um sistema autônomo é um sistema de equações diferenciais que não depende explicitamente da variável independente. Quando a variável independente é o tempo, um sistema autônomo pode também ser chamado de sistema invariante no tempo. Muitos sistemas físicos, nos quais a variável independente é o tempo, podem ser expressos como sistemas autônomos onde se supõe que as leis da natureza que valem agora são as mesmas que valiam no passado e que valerão no futuro.

Um sistema autônomo é um sistema de equações diferenciais da forma

$$
\frac{d}{d t} x(t)=f(x(t))
$$

onde $x=\left(x_{1}, x_{2}, x_{3}, \ldots, x_{n}\right)^{T} \in \mathbb{R}^{n},\left(f_{1}(x), f_{2}(x), \ldots, f_{n}(x)\right)^{T}$ com $f_{i}: \mathbb{R}^{n} \rightarrow \mathbb{R}$ são aplicações contínuas e $t$ é a variável independente representando o tempo. Os sistemas autônomos distinguemse dos sistemas de equações diferenciais da forma

$$
\frac{d}{d t} x(t)=f(x(t), t)
$$

nos quais a derivada de $x$ depende explicitamente de $t$.

Considere

$$
x\left(t_{0}\right)=x_{0}
$$

uma condição inicial. Uma solução de (2.1) com condição inicial (2.2) é uma função $x, x: \mathbb{R} \rightarrow \mathbb{R}^{n}$, $x \in \mathcal{C}^{1}$, que satisfaz (2.1) e (2.2). As equações (2.1) e (2.2) são conhecidas como problema de Cauchy. O vetor $x(t)$ é chamado de vetor de estados porque descreve o estado do sistema (2.1) no instante $t$. $\mathrm{O}$ espaço $n$-dimensional $\mathbb{R}^{n}$ chama-se espaço de estados. A representação gráfica de $(x(t), t)$ no espaço de estados estendido $\mathbb{R}^{n} \times \mathbb{R}$ chama-se curva integral. A projeção da curva integral no espaço de estados chama-se trajetória do sistema que passa pelo ponto $x\left(t_{0}\right)=x_{0}$. A solução geral $x(t)$ do sistema de equações diferenciais (2.1) depende de uma constante cujo valor só pode ser determinado pela imposição de uma condição adicional, como a condição (2.2) por 
exemplo. Se a condição inicial foi dada e a constante calculada, então a solução chama-se solução particular. Se a condição inicial não foi dada e a constante está indeterminada então a solução chama-se solução geral.

\subsection{Soluções de equilíbrio}

Uma solução $x^{*}(t)$ do sistema autônomo (2.1) é chamada de solução de equilíbrio se não depende de $t$, quer dizer, se $x^{*}(t)=x^{*}$, com $x^{*} \in \mathbb{R}^{n}$ constante. Como a solução de equilíbrio $x^{*}(t)$ não depende de $t$, temos

$$
\frac{d}{d t} x^{*}(t)=0
$$

Isto, em conjunto com o fato de que $x^{*}$ é um ponto de equilibro do sistema de equações diferenciais (2.1), implica que

$$
f\left(x^{*}\right)=0 .
$$

Resumindo, podemos definir solução de equilíbrio como segue.

Definição: Uma solução $x^{*}(t)=x^{*} \in \mathbb{R}^{n}$ do sistema de equações diferenciais (2.1) é uma solução de equilíbrio se $x^{*}$ é tal que $f\left(x^{*}\right)=0$.

Quer dizer, as soluções de equilíbrio do sistema de equações diferenciais (2.1) são zeros de $f(\cdot)$.

\subsection{Estabilidade de uma solução de equilíbrio}

Soluções de equilíbrio podem ser classificadas como estáveis ou instáveis.

Definição: Uma solução de equilíbrio $x^{*}$ do sistema (2.1) é dita Lyapunov estável se, para todo $\varepsilon>0$, existe $\delta=\delta(\varepsilon)>0$ tal que toda solução $x(t)$ que satisfaça $\left\|x\left(t_{0}\right)-x^{*}\right\|<\delta$ também satisfaz $\left\|x(t)-x^{*}\right\|<\varepsilon$ para todo $t>t_{0}$.

Conceitualmente, uma solução de equilíbrio é Lyapunov estável se todas as soluções de um sistema dinâmico que começam suficientemente perto da solução de equilíbrio (a uma distância menor que $\delta$ ) ficam perto do ponto de equilíbrio para sempre (a uma distância menor que $\varepsilon$ ). Se as soluções que começam perto da solução de equilíbrio, não apenas permanecem perto para sempre mas convergem à solução de equilíbrio, então ela é dita assintoticamente estável.

Definição: Uma solução de equilíbrio $x^{*}$ é dita assintoticamente estável se $x^{*}$ é Lyapunov estável e existe $\delta>0$ tal que para toda solução $x(t)$ que satisfaz $\left\|x\left(t_{0}\right)-x^{*}\right\|<\delta$ também satisfaz $\lim _{t \rightarrow \infty} x(t)=x^{*}$.

Soluções de equilíbrio que não são nem Lyapunov estáveis nem assintoticamente estáveis são ditas instáveis.

\subsection{Linearização ao redor de uma solução de equilíbrio}

Seja $x^{*}$ uma solução de equilíbrio do sistema de equações diferenciais (2.1). Supondo que $f \in \mathcal{C}^{2}$, expandindo (2.1) numa série de Taylor ao redor de $x^{*}$ e conservando apenas o termo linear, obtemos o modelo afim

$$
\frac{d}{d t} y(t) \approx f\left(x^{*}\right)+J_{f}\left(x^{*}\right)\left(y(t)-x^{*}\right)
$$


ou, equivalentemente, chamando de $\hat{s}(t)=y(t)-x^{*}$ ao incremento,

$$
\frac{d}{d t} \hat{s}(t) \approx f\left(x^{*}\right)+J_{f}\left(x^{*}\right) \hat{s}(t)
$$

onde $J_{f}(x) \in \mathbb{R}^{n \times n}$ representa o Jacobiano de $f(x)=\left(f_{1}(x), f_{2}(x), \ldots, f_{n}(x)\right)^{T}$ e define-se como:

$$
J_{f}(x)=\left(\begin{array}{cccc}
\frac{\partial f_{1}}{\partial x_{1}} & \frac{\partial f_{1}}{\partial x_{2}} & \cdots & \frac{\partial f_{1}}{\partial x_{n}} \\
\frac{\partial f_{2}}{\partial x_{1}} & \frac{\partial f_{2}}{\partial x_{2}} & \cdots & \frac{\partial f_{2}}{\partial x_{n}} \\
\vdots & \vdots & \ddots & \vdots \\
\frac{\partial f_{n}}{\partial x_{1}} & \frac{\partial f_{n}}{\partial x_{2}} & \cdots & \frac{\partial f_{n}}{\partial x_{n}}
\end{array}\right)=\left(\begin{array}{c}
\nabla f_{1}(x)^{T} \\
\nabla f_{2}(x)^{T} \\
\vdots \\
\nabla f_{n}(x)^{T}
\end{array}\right) .
$$

Como $x^{*}$ é uma solução de equilíbrio, temos que $f\left(x^{*}\right)=0$. Em consequência, uma solução aproximada de (2.4) pode ser calculada resolvendo

$$
\frac{d}{d t} \hat{s}(t)=J_{f}\left(x^{*}\right) \hat{s}(t)
$$

que é um sistema diferencial linear com coeficientes constantes $J_{f}\left(x^{*}\right)$. Veremos a seguir que os autovalores da matriz constante $J_{f}\left(x^{*}\right)$ fornecem informação local sobre a estabilidade da solução de equilíbrio $x^{*}$. A informação será local pois a aproximação em $(2.4)$ é válida apenas para $\hat{s}(t) \approx 0$, quer dizer, $y(t)=x^{*}+\hat{s}(t)$ próximo de $x^{*}$.

A solução particular $\hat{s}$ de $(2.5)$ que satisfaz a condição inicial $\hat{s}\left(t_{0}\right)=\hat{s}_{0}$ é dada por

$$
\hat{s}(t)=e^{\left(t-t_{0}\right) J_{f}\left(x^{*}\right)} \hat{s}\left(t_{0}\right)
$$

onde

$$
e^{\left(t-t_{0}\right) J_{f}\left(x^{*}\right)}=\sum_{i=1}^{\infty} \frac{\left(t-t_{0}\right)^{i}}{i !}\left[J_{f}\left(x^{*}\right)\right]^{i} .
$$

Se $J_{f}\left(x^{*}\right)$ possui $n$ autovalores distintos $\lambda_{1}, \lambda_{2}, \ldots, \lambda_{n}$ então existe uma matriz não singular $P \in$ $\mathbb{C}^{n \times n}$ tal que $D=P^{-1} J_{f}\left(x^{*}\right) P$, com $D=\operatorname{diag}\left(\lambda_{1}, \lambda_{2}, \ldots, \lambda_{n}\right)$. Como $J_{f}\left(x^{*}\right) P=P D$, temos que as colunas $p_{1}, p_{2}, \ldots, p_{n}$ de $P$ são os autovetores de $J_{f}\left(x^{*}\right)$ correspondentes aos autovalores $\lambda_{1}, \lambda_{2}, \ldots, \lambda_{n}$. Veja [22] para mais detalhes.

Fazendo a transformação $\hat{s}(t)=P v(t)$ em (2.5) e pré-multiplicando por $P^{-1}$ obtemos

$$
\frac{d}{d t} v(t)=D v(t)
$$

A solução particular de (2.6) que satisfaz a condição inicial $\hat{s}\left(t_{0}\right)=\hat{s}_{0}$, ou, equivalentemente, $v\left(t_{0}\right)=P^{-1} \hat{s}_{0}=v_{0}$ é dada por

$$
v(t)=e^{\left(t-t_{0}\right) D} v_{0}
$$

que reescrevendo em função de $\hat{s}(t)$ fica

$$
\hat{s}(t)=P e^{\left(t-t_{0}\right) D} P^{-1} \hat{s}_{0} .
$$

Como a matriz $e^{\left(t-t_{0}\right) D}$ em $(2.7)$ é uma matriz diagonal com entradas $e^{\left(t-t_{0}\right) \lambda_{i}}, i=1, \ldots, n$, é possível analisar $\lim _{t \rightarrow \infty} \hat{s}(t)$ em função de $\lambda_{1}, \lambda_{2}, \ldots, \lambda_{n}$, autovalores da matriz Jacobiana $J_{f}\left(x^{*}\right)$. É essa a análise conhecida como análise linear de estabilidade.

Por exemplo, se $\operatorname{Re}\left(\lambda_{i}\right)<0, \forall i$, então $e^{\left(t-t_{0}\right) \lambda_{i}} \rightarrow 0$ quando $t \rightarrow \infty$ e, em consequência, $y(t)=$ $x^{*}+\hat{s}(t) \rightarrow x^{*}$ e $\hat{s}(t) \rightarrow 0$. Quer dizer, o ponto de equilíbrio $x^{*}$ é assintoticamente estável. Análises 
similares existem para as outras possibilidades relacionadas aos valores de $\lambda_{1}, \lambda_{2}, \ldots, \lambda_{n}$ e também para o caso em que a matriz Jacobiana não é uma matriz simples (matriz com todos os autovalores diferentes). Neste último caso, a análise baseia-se na decomposição da matriz na forma canônica de Jordam. Veja [20] para mais detalhes.

De qualquer forma, a análise mencionada acima tem a limitação de estar baseada na aproximação linear (2.4) da equação diferencial (2.1). Embora a linearização ajude a determinar se uma dada solução de equilíbrio $x^{*}$ é estável ou não, ela não fornece nenhuma informação sobre o tamanho da vizinhança de $x^{*}$ dentro da qual a análise acima é válida. Conforme apresentado em [20], esta região poderia ser determinada se fosse possível construir uma função de Lyapunov para provar a estabilidade de $x^{*}$. Além disso, o estudo sobre a estabilidade de soluções de equilíbrio apresentado em [20] termina mostrando exemplos nos quais uma análise não linear é necessária para determinar se uma dada solução de equilíbrio é estável ou não. Este assunto será abordado futuramente no presente trabalho. 


\section{Capítulo 3}

\section{Perturbações condicionais não-lineares ótimas}

Dando continuidade ao nosso estudo das aplicações do SPG em Meteorologia, neste capítulo revisamos os métodos de estimativa da perturbação de maior crescimento durante a evolução de um sistema. Para tanto estudamos os conceitos de modelo tangente linear e propagador e a definição de vetor singular linear e perturbação condicional não-linear ótima. Além disso relembramos a família de métodos de Runge-Kutta e o método do Gradiente Espectral Projetado.

\subsection{Propagador e modelo tangente linear}

O conteúdo desta seção é baseado nas referências [23, 24, 25].

Dado o sistema não-linear (2.1) com condição inicial (2.2), chamamos de $M_{t}: \mathbb{R}^{n} \rightarrow \mathbb{R}^{n}$ à transformação que leva $x_{0}$ a $x(t)$, valor de $x$ em $t$ que satisfaz (2.1) e (2.2), quer dizer,

$$
x(t)=M_{t}\left[x\left(t_{0}\right)\right] .
$$

$M_{t}[\cdot]$ é chamado de propagador do sistema (2.1-2.2).

Consideremos uma perturbação inicial $s\left(t_{0}\right)=s_{0}$. A evolução no tempo de $x_{0}+s_{0}$ é dada por

$$
x(t)+s(t)=M_{t}\left[x\left(t_{0}\right)+s\left(t_{0}\right)\right] .
$$

De (3.1) e (3.2) temos que a evolução da perturbação $s\left(t_{0}\right)$ é dada por

$$
s(t)=M_{t}\left[x\left(t_{0}\right)+s\left(t_{0}\right)\right]-M_{t}\left[x\left(t_{0}\right)\right] .
$$

Neste ponto, para deduzir o modelo tangente linear, precisamos fazer uma pequena digressão e introduzir o método de Euler de integração numérica como forma de calcular aproximadamente $M_{t}[\cdot]$.

Dado um intervalo de tempo $\left[t_{0}, t_{f}\right]$, resolver numericamente o sistema (2.1-2.2) neste intervalo consiste em construir um conjunto finito de pontos $x_{0}=\bar{x}_{0}, \bar{x}_{1}, \ldots$ que satisfaçam de maneira aproximada a equação diferencial (2.1). Para tanto, discretiza-se o intervalo de tempo $\left[t_{0}, t_{f}\right] \mathrm{em} N$ subintervalos iguais e definem-se os instantes de tempo

$$
t_{k}=t_{k-1}+\Delta t=t_{0}+k \Delta t, k=0,1, \ldots, N
$$

onde $\Delta t=\left(t_{f}-t_{0}\right) / N$ é o tamanho do passo de integração. Para cada instante $t_{k}$ será calculado o ponto $\bar{x}_{k}$ aproximação de $x\left(t_{k}\right)$. Supondo que $f_{i}(x) \in \mathcal{C}^{1}$ e fazendo uma aproximação por série de Taylor para cada $f_{i}(x)$ do sistema (2.1), expandir $x(t)$ ao redor de $t=t_{0}$ resulta em

$$
x(t)=x\left(t_{0}\right)+x^{\prime}\left(t_{0}\right)\left(t-t_{0}\right)+O\left[\left\|t-t_{0}\right\|^{2}\right] .
$$

Como $x^{\prime}\left(t_{0}\right)=f\left(x\left(t_{0}\right)\right)$ e $\Delta t=t_{1}-t_{0}$, particularizando (3.5) para $t=t_{1}$ temos

$$
x\left(t_{1}\right)=x\left(t_{0}\right)+\Delta t f\left(x\left(t_{0}\right)\right)+O\left[\left\|t_{1}-t_{0}\right\|^{2}\right] .
$$


Desprezando os termos de ordem quadrática e superiores em (3.6) obtemos

$$
x\left(t_{1}\right) \approx x\left(t_{0}\right)+\Delta t f\left(x\left(t_{0}\right)\right) .
$$

Se definimos

$$
\bar{x}_{1}=\bar{x}_{0}+\Delta t f\left(\bar{x}_{0}\right),
$$

temos que $\bar{x}_{1} \approx x\left(t_{1}\right)$. Aplicando a mesma ideia de forma iterativa, se definimos

$$
\bar{x}_{k}=\bar{x}_{k-1}+\Delta t f\left(\bar{x}_{k-1}\right), k=1, \ldots, N,
$$

obtemos o denominado esquema padrão de Euler. Denotaremos por $\bar{M}_{t_{k}}[\cdot]$ ao propagador discreto do sistema (2.1-2.2) que, aplicando o método de Euler de $t_{0}$ até $t_{k}$ calcula $\bar{x}_{k}=\bar{M}_{t_{k}}\left[x_{0}\right]$, aproximação de $x\left(t_{k}\right)=M_{t_{k}}\left[x_{0}\right]$, para $k=1, \ldots, N$.

Voltando a dedução do modelo tangente linear, para $t=t_{1}$, trocando $M_{t}[\cdot]$ por $\bar{M}_{t}[\cdot]$ em (3.3) temos que

$$
s\left(t_{1}\right)=M_{t_{1}}\left[x\left(t_{0}\right)+s\left(t_{0}\right)\right]-M_{t_{1}}\left[x\left(t_{0}\right)\right] \approx \bar{M}_{t_{1}}\left[x\left(t_{0}\right)+s\left(t_{0}\right)\right]-\bar{M}_{t_{1}}\left[x\left(t_{0}\right)\right]=\bar{s}_{1} .
$$

De (3.8), temos que

$$
\begin{aligned}
\bar{s}_{1} & =\bar{M}_{t_{1}}\left[x\left(t_{0}\right)+s\left(t_{0}\right)\right]-\bar{M}_{t_{1}}\left[x\left(t_{0}\right)\right] \\
& =\left[x\left(t_{0}\right)+s\left(t_{0}\right)+\Delta t f\left(x\left(t_{0}\right)+s\left(t_{0}\right)\right)\right]-\left[x\left(t_{0}\right)+\Delta t f\left(x\left(t_{0}\right)\right)\right] \\
& =s\left(t_{0}\right)+\Delta t\left(f\left(x\left(t_{0}\right)+s\left(t_{0}\right)\right)-f\left(x_{0}\right)\right) .
\end{aligned}
$$

Substituindo $f\left(x\left(t_{0}\right)+s\left(t_{0}\right)\right)$ em (3.9) pela sua aproximação linear $f\left(x\left(t_{0}\right)\right)+J_{f}\left(x\left(t_{0}\right)\right) s\left(t_{0}\right)$ temos que

$$
s\left(t_{1}\right) \approx \bar{s}_{1} \approx \tilde{s}_{1}=s_{0}+\Delta t J_{f}\left(x_{0}\right) s_{0} .
$$

Quer dizer, $\tilde{s}_{1}$ é uma aproximação de $\bar{s}_{1}$ que por sua vez é uma aproximação de $s\left(t_{1}\right)$. $\bar{s}_{1}$ é uma aproximação de $s\left(t_{1}\right)$ porque calcula-se utilizando o propagador discreto no lugar do propagador verdadeiro e $\tilde{s}_{1}$ é uma aproximação de $\bar{s}_{1}$ porque calcula-se utilizando o propagador discreto mas com a $f$ substituída pela sua aproximação linear.

Definindo $D_{k}=I+\Delta t J_{f}\left(\bar{x}_{k}\right)$ é fácil ver que

$$
\tilde{s}_{k}=D_{k-1} s_{k-1}=D_{k-1} D_{k-2} \ldots D_{0} s_{0} .
$$

Se chamamos $L_{t_{k}}=D_{k-1} D_{k-2} \ldots D_{0}$ temos que a evolução da perturbação $s_{0}$ até o tempo $t_{k}$ pode ser aproximada por

$$
s\left(t_{k}\right) \approx \tilde{s}_{k}=L_{t_{k}} s_{0} .
$$

A equação (3.10) é conhecida como modelo tangente linear (TLM, do inglês Tangent Linear Model). Parece natural analisar a matriz $L_{t_{k}}$ para inferir de que forma evolui a perturbação inicial $s_{0}$ até $o$ instante de tempo $t_{k}$.

\subsection{Valores e vetores singulares lineares}

Dada uma condição inicial da forma (2.2) e um tempo final $t_{f}$, desejamos saber qual é a perturbação $s_{0}$ que maximiza a diferença entre $x\left(t_{f}\right)$ e $x\left(t_{f}\right)+s\left(t_{f}\right)$, quer dizer, que maximiza $\left\|s\left(t_{f}\right)\right\|_{2}$. Em função do exposto na seção anterior, fixando um $N$ arbitrário temos que $\tilde{s}_{N} \approx s\left(t_{N}\right)$ e resulta natural tentar achar a perturbação $s_{0}$ que maximiza $\left\|\tilde{s}_{N}\right\|_{2}$. De (3.10) temos que $\tilde{s}_{N}=L_{t_{N}} s_{0}$ e, portanto,

$$
\left\|\tilde{s}_{N}\right\|_{2}=\sqrt{s_{0}^{T} L_{t_{N}}^{T} L_{t_{N}} s_{0}}
$$

Para que o problema acima esteja bem definido, precisamos limitar nossa busca a, por exemplo, 
$s_{0} \in B=\left\{s \in \mathbb{R}^{n} \mid\|s\|_{2}=1\right\}$. Desta forma, o problema a ser resolvido é

$$
\operatorname{Maximizar}_{s_{0}} s_{0}^{T} L_{t_{N}}^{T} L_{t_{N}} s_{0} \text { sujeita a }\left\|s_{0}\right\|_{2}^{2}=1 .
$$

As condições KKT de (3.11) são:

$$
\begin{aligned}
2 L_{t_{N}}^{T} L_{t_{N}} s_{0}-\lambda 2 s_{0} & =0 \\
\left\|s_{0}\right\|_{2}^{2} & =1
\end{aligned}
$$

Claramente os pares $\left(s_{0}, \lambda\right)$ que satisfazem (3.12) são autopares da matriz normal $L_{t_{N}}^{T} L_{t_{N}}$ tais que $\left\|s_{0}\right\|_{2}=1$ e é fácil ver que o ponto estacionário $\left(s_{0}, \lambda\right)$ que maximiza a função objetivo de (3.11) é aquele tal que $\lambda$ é o maior autovalor da matriz normal $L_{t_{N}}^{T} L_{t_{N}}$. Logo, interessa calcular o maior valor singular da matriz $L_{t_{N}}$ e seu vetor singular associado. Esta abordagem é conhecida como método dos valores e vetores singulares lineares.

\subsection{Perturbações condicionais não-lineares ótimas}

O conteúdo desta Seção está baseado no trabalho [3].

A abordagem dos valores e vetores singulares lineares baseia-se num propagador discreto que utiliza o método de integração numérica de Euler e uma aproximação linear da função $f$. Sendo assim, fica claro que essa abordagem serve apenas para aproximar a evolução de perturbações em períodos curtos de tempo. Daí surge a necessidade de um método que leve em conta as não linearidades do sistema de equações diferenciais.

Recapitulando, temos de (3.3) que a evolução de uma perturbação $s\left(t_{0}\right)=s_{0}$ é dada por

$$
s(t)=M_{t}\left[x\left(t_{0}\right)+s\left(t_{0}\right)\right]-M_{t}\left[x\left(t_{0}\right)\right] .
$$

Idealmente, dados $t_{f}$ e $\delta>0$, desejamos calcular $s_{0}$ solução de

$$
\operatorname{Maximizar}_{s_{0}} \frac{1}{2}\left\|s\left(t_{f}\right)\right\|_{2}^{2} \text { sujeita a }\left\|s_{0}\right\|_{2} \leq \delta .
$$

A função objetivo de (3.14) é dada por

$$
\left\|s\left(t_{f}\right)\right\|_{2}=W\left(s_{0}\right)=\frac{1}{2}\left\|M_{t}\left[x_{0}+s_{0}\right]-M_{t}\left[x_{0}\right]\right\|_{2}^{2} .
$$

Se o propagador $M_{t}$ em (3.15) for substituído por um propagador discreto então o problema (3.14) torna-se um problema de programação não-linear. Define-se perturbação condicional não-linear ótima como a solução desse problema de programação não-linear (que depende de $\delta$, do propagador discreto e seus parâmetros). A escolha da norma Euclidiana na restrição do problema (3.14) justificase pelo fato de que se o propagador discreto escolhido for o Método de Euler, $\delta=1$ e a função $f$ em (2.1) for linear, então o problema (3.15) coincide com o problema (3.11).

\subsection{Método de Runge-Kutta}

O problema (3.14) com função objetivo (3.15) é transformado num problema de programação matemática utilizando um esquema de discretização para $M[\cdot]$ em (3.14). Neste trabalho, dados $K \in\{1,2,4\}, \alpha \in \mathbb{R}^{K}$ e $\beta \in \mathbb{R}^{K-1}$, definimos $x_{i+1}^{0}$, evolução de $x_{0}$ até o instante de tempo $t_{i+1}$ dado pela família de métodos de Runge-Kutta conforme [26]. Consideremos

$$
x_{i+1}^{0}=x_{i}^{0}+\Delta t \sum_{j=0}^{K+1} \alpha_{j} f\left(x_{i}^{j}\right),
$$


onde

$$
x_{i}^{j+1}=x_{i}^{0}+\beta_{j} \Delta t f\left(x_{i}^{j}\right), j=0, \ldots, K-2,
$$

para $i=0, \ldots, N-1$. Aqui, $x_{i}^{0} \equiv x_{i}, t_{i}^{0} \equiv t_{i}, t_{i}^{j} \equiv t_{i}+\beta_{j-1} \Delta t$ e $x_{i}^{0} \in R^{n}$ são vetores auxiliares.

\begin{tabular}{c|c|c|c}
\hline Método de Integração & $\mathbf{K}$ & $\alpha$ & $\beta$ \\
\hline Runge-Kutta ordem 1 ou Euler & 1 & $\alpha_{0}=1$ & - \\
Runge-Kutta ordem 2 ou Euler modificado & 2 & $\alpha_{0}=0, \alpha_{1}=1$ & $\beta_{0}=0.5$ \\
Runge-Kutta ordem 2 & 2 & $\alpha_{0}=\alpha_{1}=0.5$ & $\beta_{0}=1$ \\
Runge-Kutta ordem 4 & 4 & $\alpha_{0}=\alpha_{3}=1 / 6$ & $\beta_{0}=\beta_{1}=0.5$ \\
& & $\alpha_{1}=\alpha_{2}=1 / 3$ & $\beta_{2}=1$ \\
\hline
\end{tabular}

Tabela 3.1: Exemplos de métodos da familia Runge-Kutta.

A Tabela 3.1 mostra alguns exemplos de métodos da família de Runge-Kutta. Daqui em diante denominaremos $M_{t_{f}}^{R K(N, K, \alpha, \beta)}\left[x_{0}\right]$ a evolução de $x_{0}$ até o instante de tempo $t_{f}$ calculada pelo método de Runge-Kutta com $N$ passos de integração e parâmetros $K, \alpha$ e $\beta$. Logo, se definirmos

$$
\bar{W}\left(s_{0}\right)=\frac{1}{2}\left\|M_{t_{f}}^{R K(N, K, \alpha, \beta)}\left[x_{0}+s_{0}\right]-M_{t_{f}}^{R K(N, K, \alpha, \beta)}\left[x_{0}\right]\right\|_{2}^{2}
$$

obtemos o problema de programação não-linear que resulta de substituir $M_{t}[\cdot]$ por $M_{t_{f}}^{R K(N, K, \alpha, \beta)}$ em (3.15):

$$
\text { Maximizar } \bar{W}\left(s_{0}\right) \text { sujeita a }\left\|s_{0}\right\|_{2}^{2} \leq \delta^{2} .
$$

À perturbação obtida através da resolução do problema (3.19) chamaremos de $\hat{s}\left(t_{0}\right)$ ou simplesmente $\hat{s}$.

\subsection{Método do gradiente espectral projetado}

Nesta seção estudaremos o método do Gradiente Espectral Projetado, utilizado no cálculo das CNOPs introduzidas na Seção 3.3. O texto a seguir está fortemente baseado no trabalho [27].

O SPG trata-se de uma implementação em Fortran 77 para o algoritmo do gradiente espectral projetado (SPG, do inglês spectral projected gradient). O método SPG aplica-se a problemas da forma

$$
\min f(x) \text { sujeito a } x \in \Omega,
$$

onde $\Omega$ é um conjunto convexo fechado em $\mathbb{R}^{n}$. Assume-se que $f$ está definida e tem derivadas parciais contínuas em um conjunto aberto que contém $\Omega$. O utilizador do pacote deve fornecer sub-rotinas que calculam a função $f(x)$, o gradiente $\nabla f(x)$ e projeções de um ponto arbitrário $x$ em $\Omega$. Informação sobre a matriz Hessiana não é requerida e os requisitos de memória são mínimos. Portanto, o algoritmo é apropriado para problemas convexos de otimização de grande escala com projeções baratas sobre o conjunto viável. Note que o algoritmo é também adequado para problemas irrestritos simples ao escolher $\Omega=\mathbb{R}^{n}$. O algoritmo é inteiramente descrito em [28], onde o método é combinado com duas novas características em otimização.

\subsubsection{Algoritmo SPG}

Dado $\hat{x} \in \mathbb{R}^{n}$ podemos definir $P_{\Omega}(\hat{x})$ como sendo a projeção com respeito a uma dada norma $\|\cdot\|$ no conjunto convexo $\Omega$, ou seja, $P_{\Omega}(\hat{x})=\operatorname{argmin}_{x \in \Omega}\|x-\hat{x}\|$. Também denotamos $g(x)=\nabla f(x)$. $\mathrm{O}$ algoritmo inicia com $x_{0} \in \mathbb{R}^{n}$ e utiliza um inteiro $m \geq 1$, um parâmetro $\alpha_{\min } \geq 0$, os parâmetros de proteção $0 \leq \sigma_{1} \leq \sigma_{2} \leq 1$. Inicialmente $\alpha \in\left[\alpha_{\min }, \alpha_{\max }\right]$ é uma escolha arbitrária.

O Algorítimo 1 é baseado na direção do gradiente espectral projetado $P_{\Omega}\left(x_{k}-\alpha_{k} g\left(x_{k}\right)\right)-x_{k}$, onde 


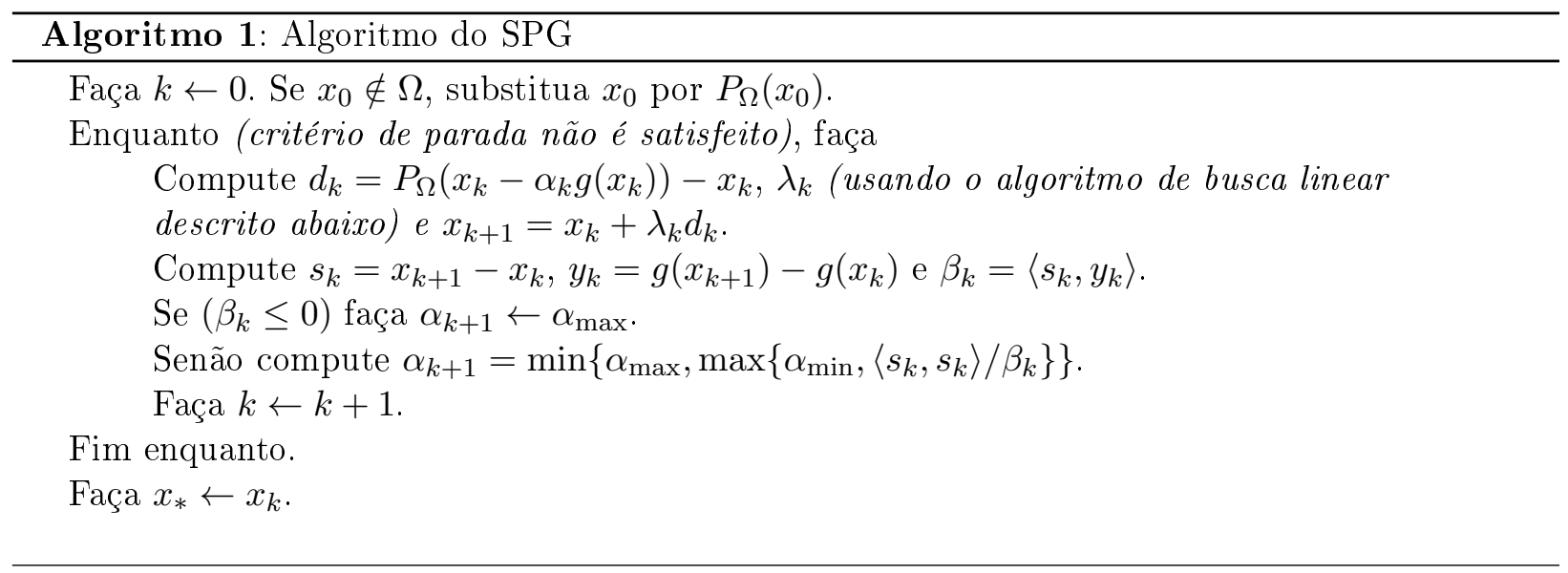

$\alpha_{k}$ é o "quociente inverso de Rayleigh" $\frac{\left\langle s_{k-1}, s_{k-1}\right\rangle}{\left\langle s_{k-1}, y_{k-1}\right\rangle}$ com salvaguardas. (Observe que $\frac{\left\langle s_{k-1}, s_{k-1}\right\rangle}{\left\langle s_{k-1}, y_{k-1}\right\rangle}$ é o quociente de Rayleigh correspondendo a matriz Hessiana média $\left.\int_{0}^{1} \nabla^{2} f\left(x_{k-1}+t s_{k-1}\right) d t\right)$.

A busca linear descrita no algoritmo 2 é baseada em uma interpolação quadrática salvaguardada. O procedimento de salvaguarda atua quando o mínimo $\lambda_{\text {temp }}$ da quadrática unidimensional interpolante está fora do intervalo $\left[\sigma_{1}, \sigma_{2} \lambda\right]$, e não quando está fora de $\left[\sigma_{1} \lambda, \sigma_{2} \lambda\right]$ como é usualmente implementado. Isto significa que, quando a interpolação tende a rejeitar $90 \%$ (para $\sigma_{1}=0.1$ ) do intervalo de busca original $([0,1])$, julgamos que sua previsão não é confiável e preferimos a bisseção mais conservadora. Este procedimento acaba sendo mais eficiente que o procedimento clássico.

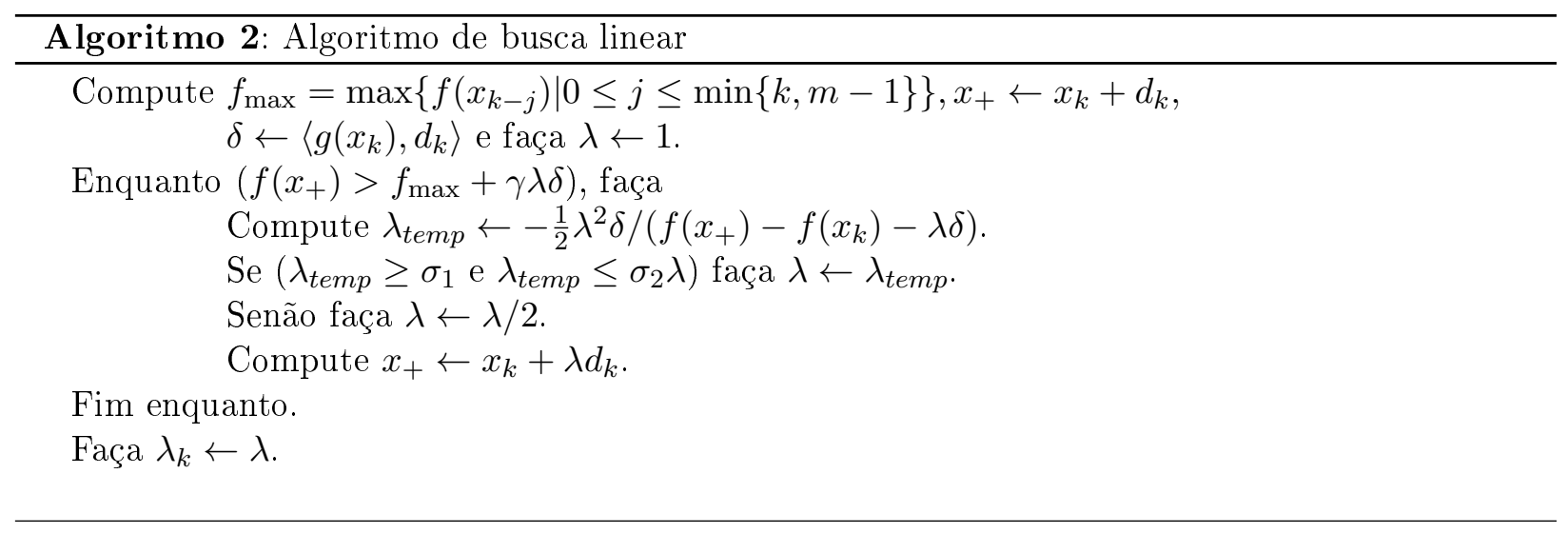

No caso de rejeição do primeiro ponto de teste, os próximos são computados ao longo da mesma direção. Como consequência, a operação de projeção é realizada apenas uma vez por iteração.

Usamos o critério de convergência dado por

$$
\left\|P_{\Omega}\left(x_{k}-g\left(x_{k}\right)\right)-x_{k}\right\|_{\infty} \leq \varepsilon .
$$

Além disso, o algoritmo para quando o número de iterações excede maxit.

Desta forma, utilizando o SPG e fornecendo o gradiente exato da função $f$ conforme realizado em [26], obtivemos as CNOPs através de experimentos que abordaremos nos próximos capítulos. 


\section{Capítulo 4}

\section{Testes preliminares}

Afim de consolidar nosso domínio sobre LSVs e CNOPs, o presente capítulo tem o objetivo de revisar a teoria proposta até o momento e analisar a sua aplicação num exemplo simples. Para tanto, consideramos o sistema de equações diferenciais não-lineares de Lorenz de 1963 [29] e calculamos a suas LSVs da forma em que foi feito em [30] (veja também [31, 32, 33]) e as CNOPs.

\subsection{Modelo de Lorenz de 1963}

O sistema de Lorenz (para maiores informações veja [29, 30, 31, 32, 33, 34, 35]) tem a forma

$$
\left(\begin{array}{c}
\frac{d x_{1}}{d t} \\
\frac{d x_{2}}{d t} \\
\frac{d x_{3}}{d t}
\end{array}\right)=\left(\begin{array}{l}
f_{1}(x) \\
f_{2}(x) \\
f_{3}(x)
\end{array}\right)=\left(\begin{array}{ccc}
-\sigma & \sigma & 0 \\
r & -1 & -x_{1} \\
x_{2} & 0 & -b
\end{array}\right)\left(\begin{array}{l}
x_{1} \\
x_{2} \\
x_{3}
\end{array}\right) .
$$

Conforme [32], o modelo (4.1) é composto por três equações diferenciais que representam a evolução temporal da variável de estado $x=\left(x_{1}, x_{2}, x_{3}\right)^{T}$. Em (4.1), $\sigma, r$ e $b$ são três parâmetros positivos [35]. As equações foram derivadas de um modelo de convecção de fluído e provém da ideia de uma célula de fluído bidimensional aquecida na base e resfriada no topo. O movimento convectivo resultante é modelado por uma equação parcial diferencial que, após simplificações, resulta em (4.1). Maiores detalhes serão fornecidos mais adiante, onde retomaremos o estudo do modelo no contexto do problema de previsibilidade.

Dados uma condição inicial $x\left(t_{0}\right)=x_{0}$, uma perturbação $s\left(t_{0}\right)=s_{0}$ sobreposta a $x_{0}$, um tempo final $t_{f}$ e um número de passos $N$ para o método de Euler, desejamos escrever o TLM de (4.1) que provê uma aproximação $\tilde{s}$ para $s\left(t_{k}\right)$, evolução de $s_{0}$ até o tempo $t_{k}=t_{0}+k \Delta t \operatorname{com} \Delta t=\left(t_{f}-t_{0}\right) /(N-1)$.

O Jacobiano de $f(x)$ em (4.1) tem a forma

$$
J_{f}(x)=\left(\begin{array}{lcr}
-\sigma & \sigma & 0 \\
\left(r-x_{3}\right) & -1 & -x_{1} \\
x_{2} & x_{1} & -b
\end{array}\right)
$$

e conforme (3.10), o TLM de (4.1) é dado por

$$
\tilde{s}_{k}=L_{t_{k}} s_{0}=D_{k-1} D_{k-2} \ldots D_{0} s_{0}
$$

onde

$$
D_{k}=\left[\left(\begin{array}{lll}
1 & 0 & 0 \\
0 & 1 & 0 \\
0 & 0 & 1
\end{array}\right)+\Delta t\left(\begin{array}{lrr}
-\sigma & \sigma & 0 \\
\left(r-x_{3}^{k}\right) & -1 & -x_{1}^{k} \\
x_{2}^{k} & x_{1}^{k} & -b
\end{array}\right)\right] .
$$


O cálculo do LSV de (4.1) para $x_{0}, s_{0}, t_{0}, t_{f}$ e $N$ dados consiste em calcular o maior valor singular e o vetor singular associado à matriz $L_{t_{N}}^{T} L_{t_{N}}$ conforme definida em (4.2).

\subsection{Experimentos numéricos}

Consideremos o sistema de Lorenz (4.1) com constantes $\sigma=10, b=8 / 3$ e $r=28$, conforme apresentado em [30, 33]. Consideremos as opções $x_{0}^{\prime}=(1.0,1.0,1.0)^{T}$ e $x_{0}^{\prime \prime}=(\sqrt{b(r-1)}, \sqrt{b(r-1)}, r-1)^{T}$ para a condição inicial $x\left(t_{0}\right)=x_{0}$. Na integração numérica de (4.1) consideraremos $\Delta t=0.01 \mathrm{e}$ $N=10$. Cada um destes dois problemas será analisado utilizando as abordagens LSV e CNOP.

Para o cálculo dos LSVs utilizamos a rotina de decomposição de valores singulares lineares gsl_linalg_SV_decomp da biblioteca GSL [36]. Para o cálculo das CNOPs consideraremos o problema (3.15) com o método de Runge-Kutta com $K=4$ e $\delta=1$. Implementamos a nossa própria rotina de Runge-Kutta, uma vez que esta é parte integrante da função (3.18) objetivo do problema de programação não-linear (3.19) que será resolvido e, portanto, faz-se necessário também calcular as suas derivadas. Por conta disso, sacrificamos a possibilidade de utilizar rotinas prontas que implementassem métodos mais sofisticados de integração numérica incluindo, por exemplo, passos múltiplos. O problema (3.19) é um problema de maximização de uma função contínua e diferenciável num conjunto convexo e para resolvê-lo utilizaremos o método SPG. Como o SPG é um método capaz de encontrar pontos estacionários de (3.19), precisamos de alguma estratégia para aumentar as chances de encontrar uma solução global. Nosso primeiro experimento tem por objetivo analisar algumas variantes de métodos estocásticos de otimização global baseados em multistart [37].

A primeira tentativa para aumentar as chances do SPG encontrar uma solução global de (3.19) consistiu em sortear $p$ pontos aleatórios uniformemente distribuídos na bola $B_{\delta}=\left\{x \in \mathbb{R}^{3} \mid\|x\|_{2} \leq\right.$ $\delta$ e utilizar aquele com melhor (maior) valor de função objetivo como ponto inicial de uma única chamada ao SPG. Chamaremos essa estratégia de M1 daqui em diante. Numa segunda tentativa, que chamaremos de M2, sorteamos $p$ pontos e utilizamos cada um deles como ponto inicial do SPG. Neste caso consideramos como solução global o melhor de todos os pontos devolvidos nas $p$ chamadas ao SPG. Em todos os casos utilizamos $\varepsilon=10^{-8}$ no critério de parada (3.21) do SPG e maxit $=5.000$ iterações.

Consideramos inicialmente a aplicação da estratégia M1 nos dois problemas com $N=10$ e condições iniciais $x_{0}^{\prime}$ e $x_{0}^{\prime \prime}$, variando $p \in\left\{10,10^{2}, 10^{3}, 10^{4}, 10^{5}, 10^{6}\right\}$. Rodamos a estratégia M1 cem vezes e analisamos a aproximação do maximizador global encontrada em cada uma das cem rodadas. A Tabela (4.1) e as Figuras 4.1(a-f) e 4.2(a-f) mostram os resultados obtidos. As figuras apresentam, para cada uma das 100 tentativas, o valor da função objetivo no ponto inicial e no ponto final (aproximação do maximizador). É possível observar na Figura 4.1(f) que apenas no caso $p=10^{6}$ a estratégia M1 encontra sempre o que parece ser a solução global do problema. Em todos os outros casos, quer dizer, com $p=\left\{10,10^{2}, \ldots, 10^{5}\right\}$, as Figuras $4.1(\mathrm{a}-\mathrm{e})$ mostram que várias das rodadas terminam num ponto estacionário que definitivamente não é um maximizador global do problema. Resultados similares são obtidos no problema com condição inicial $x_{0}^{\prime \prime}$ e a Figura 4.2(e) mostra que com $p=10^{5}$ a estratégia M1 encontra o que parece ser a solução global em todas as cem tentativas. De qualquer forma, o experimento sugere que o melhor dentre um número muito grande de pontos aleatórios deve ser utilizado como ponto inicial do SPG para que este não convirja a pontos estacionários não globais.

Consideramos agora a aplicação da estratégia M2 aos mesmos dois problemas variando $p \in$ $\{1,5,10,15\}$. As Figuras 4.3(a-b) mostram os resultados para os problemas com condições iniciais $x_{0}^{\prime}$ e $x_{0}^{\prime \prime}$, respectivamente. A Tabela 4.2 apresenta os resultados percentuais. Os gráficos representam a aproximação do máximo encontrado pela estratégia M2 para cada valor de $p$ e sugerem que para $p \geq 10$ a estratégia sempre encontra o que parece ser o máximo global dos dois problemas. Cabe destacar que a aproximação encontrada coincide com a encontrada pela estratégia M1 com $p=10^{6}$ sugerindo fortemente que as estratégias efetivamente resolvem globalmente os problemas 
considerados nestes experimentos.

\begin{tabular}{ccccccc}
\hline & \multicolumn{6}{c}{ Porcentagem de erros da estratégia M1 } \\
\hline & $p=10$ & $p=10^{2}$ & $p=10^{3}$ & $p=10^{4}$ & $p=10^{5}$ & $p=10^{6}$ \\
\hline$x^{\prime}$ & $43 \%$ & $52 \%$ & $41 \%$ & $33 \%$ & $10 \%$ & $0 \%$ \\
$x^{\prime \prime}$ & $48 \%$ & $52 \%$ & $37 \%$ & $16 \%$ & $0 \%$ & - \\
\hline
\end{tabular}

Tabela 4.1: Número de vezes em que o máximo obtido pela estratégia M1 não foi o máximo global esperado para diferentes valores de $p$.

\begin{tabular}{|c|c|c|c|c|}
\hline \multicolumn{5}{|c|}{ Porcentagem de erros da estratégia M2 } \\
\hline & $p=1$ & $p=5$ & $p=10$ & $p=15$ \\
\hline$x^{\prime}$ & $51 \%$ & $3 \%$ & $0 \%$ & $\overline{0}$ \\
\hline$x^{\prime \prime}$ & $45 \%$ & $8 \%$ & $0 \%$ & $0 \%$ \\
\hline
\end{tabular}

Tabela 4.2: Número de vezes em que o máximo obtido pela estratégia M2 não foi o máximo global esperado para diferentes valores de $p$.

Os experimentos acima descritos servem para tomar a decisão de utilizar o SPG no contexto da estratégia M2 com $p=10$ nos experimentos a seguir para o cálculo das CNOPs. Chamaremos este método de Multistart-SPG daqui em diante.

Utilizando portanto a estratégia Multistart-SPG para o cálculo das CNOPs realizamos alguns testes comparativos afim de verificar como LSVs e CNOPs se comportam para diferentes períodos de integração. Para tanto, calculamos as CNOPs e LSVs para $N \in\{1,2,3, \ldots, 40\}$ a partir das condições iniciais $x_{0}^{\prime}$ e $x_{0}^{\prime \prime}$. As Figuras $4.4(\mathrm{a}-\mathrm{b})$ mostram os valores da função objetivo $\bar{W}\left(s_{0}^{L S V\left(t_{f}\right)}\right)$ e $\bar{W}\left(s_{0}^{C N O P\left(t_{f}\right)}\right)$ com relação a $N \in\{1,2,3, \ldots, 40\}$. A Tabela 4.4 exibe os valores das CNOPs e LSVs mostrados nas Figuras 4.4(a-b). Observa-se que para $N \leq 10$ os valores de $\bar{W}\left(s_{0}^{L S V\left(t_{f}\right)}\right)$ e $\bar{W}\left(s_{0}^{C N O P\left(t_{f}\right)}\right)$ são próximos e que, como esperado, em todos os casos vale que $\bar{W}\left(s_{0}^{L S V\left(t_{f}\right)}\right) \leq \bar{W}\left(s_{0}^{C N O P\left(t_{f}\right)}\right)$.

Utilizando Multistart-SPG e considerando que os métodos são métodos de passo fixo, verificamos qual o impacto da escolha do método de integração nos ótimos da função objetivo. Para tanto, resolvemos os problemas com condição inicial $x_{0}=x_{0}^{\prime}$ e $x_{0}=x_{0}^{\prime \prime}$ obtendo os valores ótimos de função objetivo detalhados na Tabela 4.3. A tabela mostra que os valores ótimos variam conforme varia o valor de $K$. Concluímos então que a definição de CNOP é dependente do método de integração numérica escolhido.

\begin{tabular}{c|cc|cc}
\hline & $x_{0}^{\prime}$ & \multicolumn{2}{c}{$x_{0}^{\prime \prime}$} \\
\hline$K$ & $s_{0}^{C N O P\left(t_{f}\right)}$ & $\bar{W}\left(s_{0}^{L S V\left(t_{f}\right)}\right)$ & $s_{0}^{C N O P\left(t_{f}\right)}$ & $\bar{W}\left(s_{0}^{L S V\left(t_{f}\right)}\right)$ \\
\hline Euler & $(-0.775806,-0.630922,-0.007877)$ & 5.522466 & $(-0.752553,-0.658141,0.022690)$ & 4.530532 \\
Euler modificado & $(-0.774017,-0.633112,-0.008121)$ & 6.199503 & $(-0.750734,-0.660147,0.024581)$ & 4.896669 \\
Runge-Kutta ordem 2 & $(-0.774007,-0.633124,-0.008181)$ & 6.199409 & $(-0.750736,-0.660154,0.024327)$ & 4.894456 \\
Runge-Kutta ordem 4 & $(-0.774216,-0.632869,-0.008111)$ & 6.229745 & $(-0.750844,-0.660012,0.024846)$ & 4.901811 \\
\hline
\end{tabular}

Tabela 4.3: Resultados da integração para $N=10$ partir das condições iniciais $x_{0}^{\prime}$ e $x_{0}^{\prime \prime}$.

Abordamos agora a questão de resolver um sistema de equações diferenciais lineares. Neste caso, pela teoria estudada, a solução das CNOPs deve coincidir com o resultado obtido através de LSVs. Escolhemos o sistema [21](p.268)

$$
\left(\begin{array}{c}
\frac{d x_{1}}{d t} \\
\frac{d x_{2}}{d t}
\end{array}\right)=\left(\begin{array}{cc}
4.0 & 0.25 \\
-4.0 & 4.0
\end{array}\right)\left(\begin{array}{l}
x_{1} \\
x_{2}
\end{array}\right)
$$


com condição inicial $x_{0}=(0.1,0.5)^{T}, \Delta t=0.01, t_{f}=10 \Delta t$ e $\delta=1.0$. Chamaremos de $s_{0}^{L S V\left(t_{f}\right)}$ a aproximação da perturbação de máximo crescimento até o instante $t_{f}$ calculada usando LSVs. Da mesma forma chamaremos $s_{0}^{C N O P\left(t_{f}\right)}$ a aproximação calculada usando CNOP. Como esperado, temos que $s_{0}^{L S V\left(t_{f}\right)}=s_{0}^{C N O P\left(t_{f}\right)}=(-0.7710,-0.6359)^{T}$ confirmando que o cálculo das CNOPs para um modelo linear coincide com os resultados obtidos através da abordagem linear dos LSVs. 


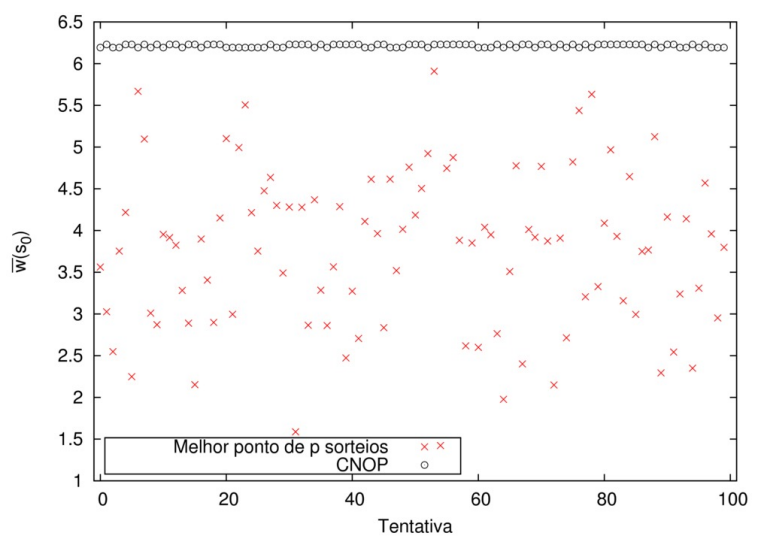

(a) $p=10$

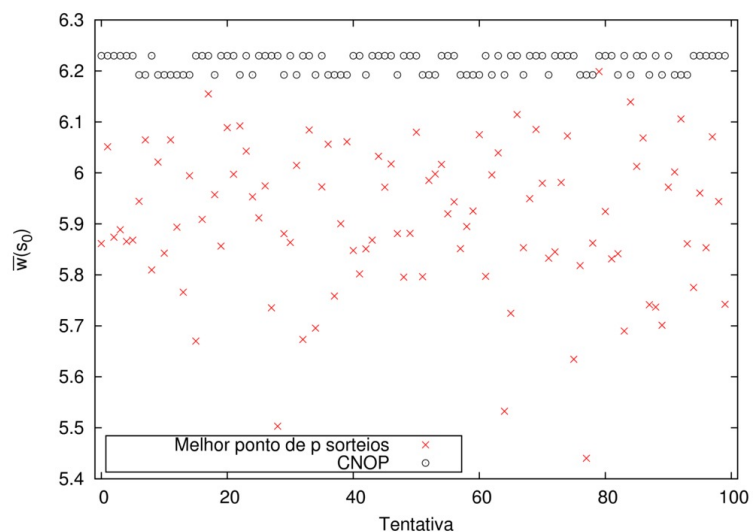

(c) $p=10^{3}$

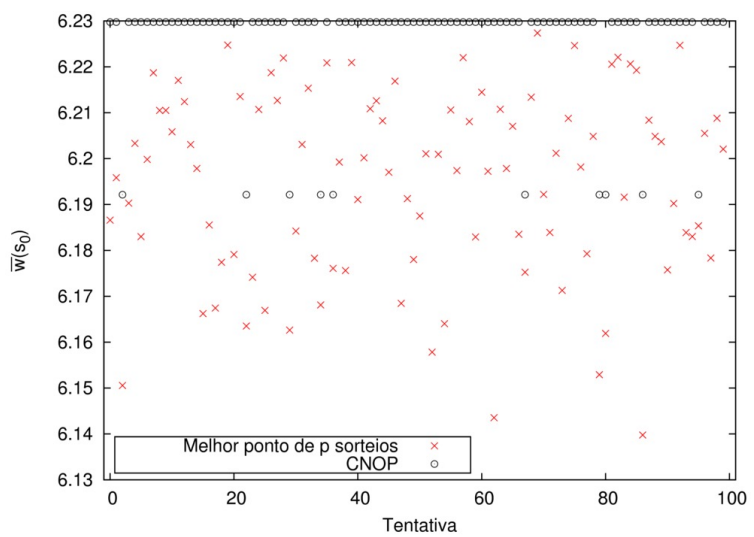

(e) $p=10^{5}$

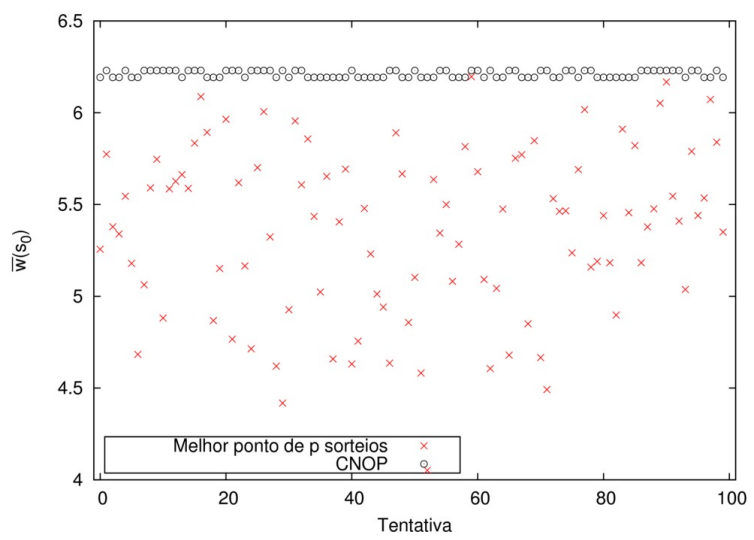

(b) $p=10^{2}$

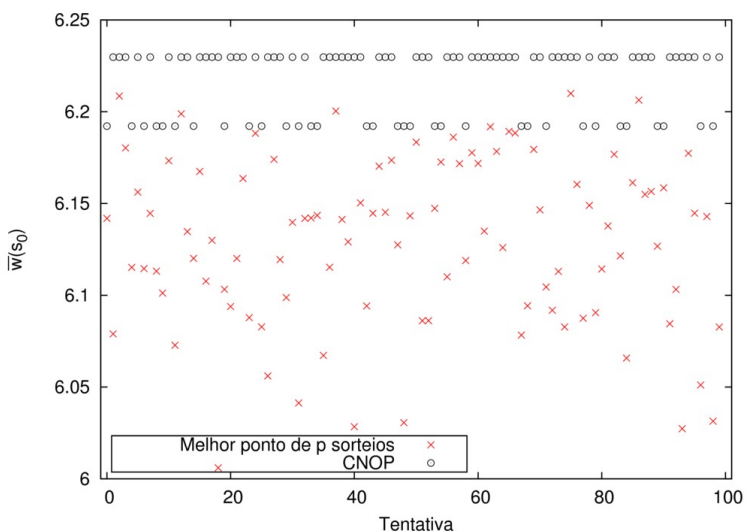

(d) $p=10^{4}$

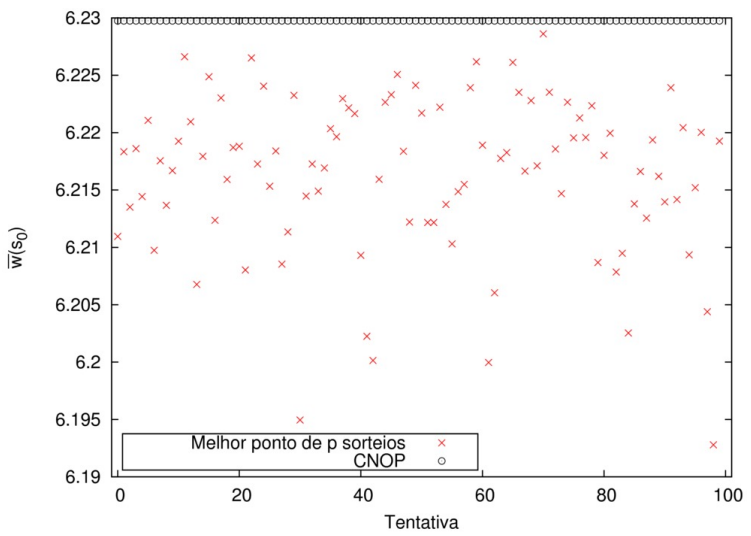

(f) $p=10^{6}$

Figura 4.1: Valores de máximo da função objetivo do problema com $N=10$ e $x_{0}=x_{0}^{\prime}$ calculados com a estratégia M1 usando diferentes valores de $p$. 


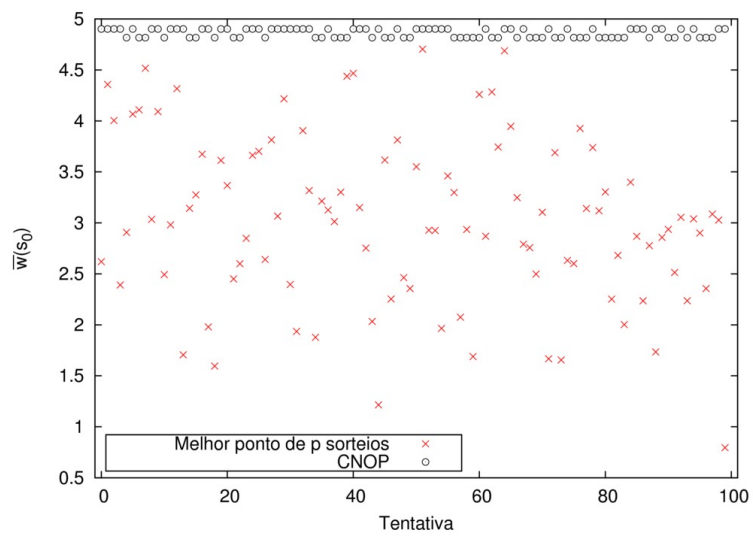

(a) $p=10$

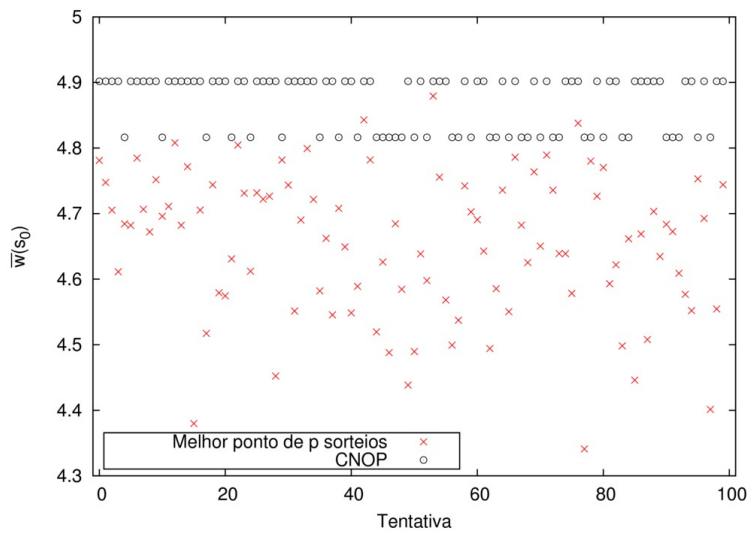

(c) $p=10^{3}$

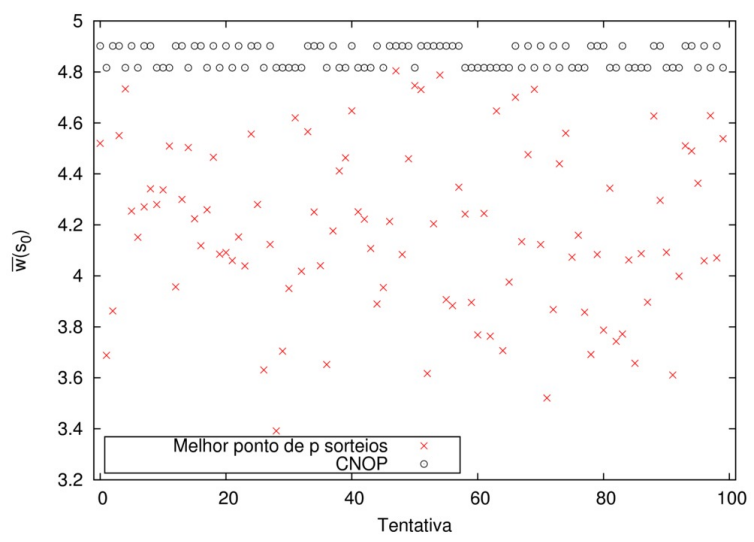

(b) $p=10^{2}$

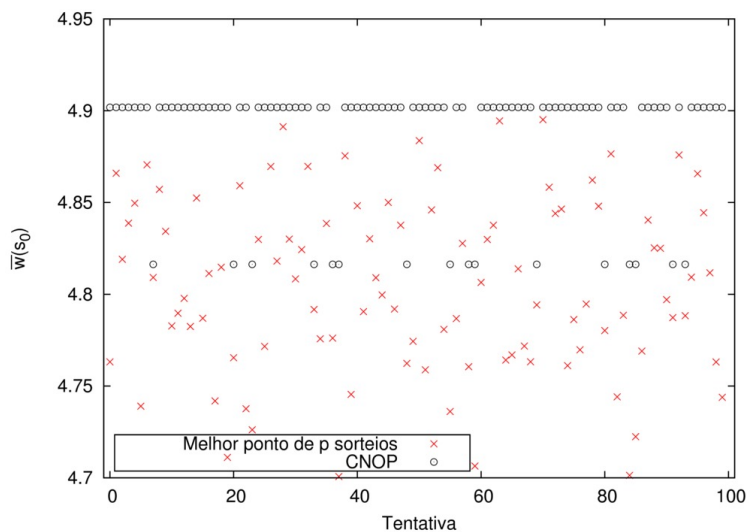

(d) $p=10^{4}$

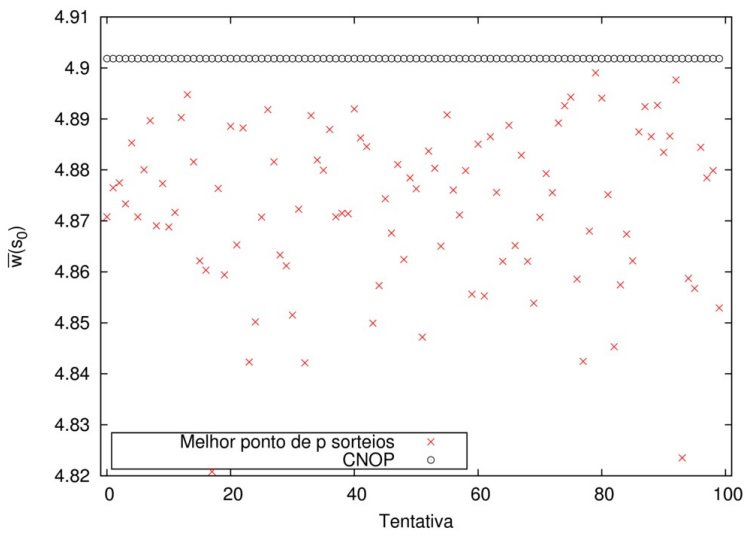

(e) $p=10^{5}$

Figura 4.2: Valores de máximo da função objetivo do problema com $N=10$ e $x_{0}=x_{0}^{\prime \prime}$ calculados com a estratégia M1 usando diferentes valores de $p$. 


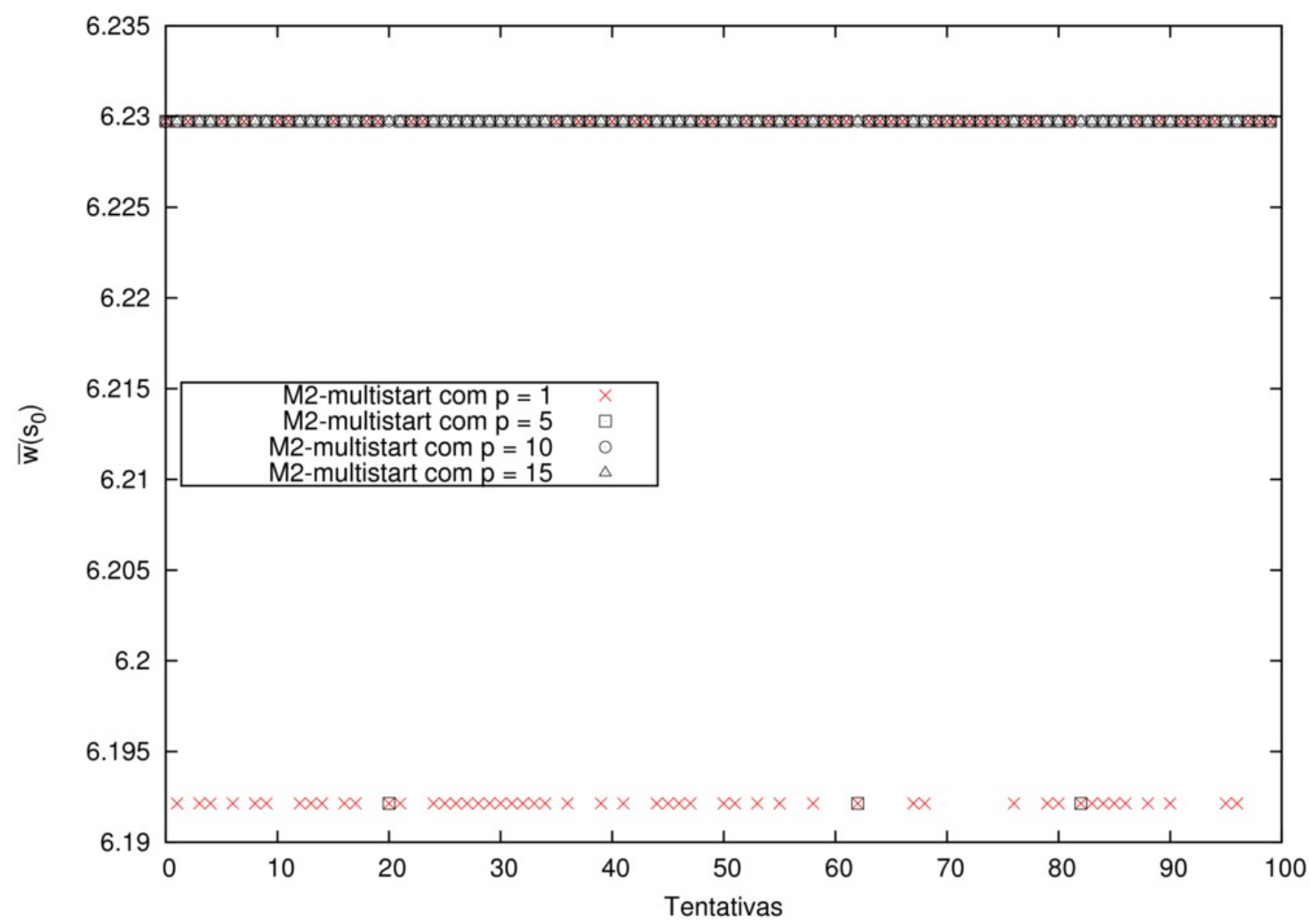

(a) Problema com $x_{0}=x_{0}^{\prime}$.

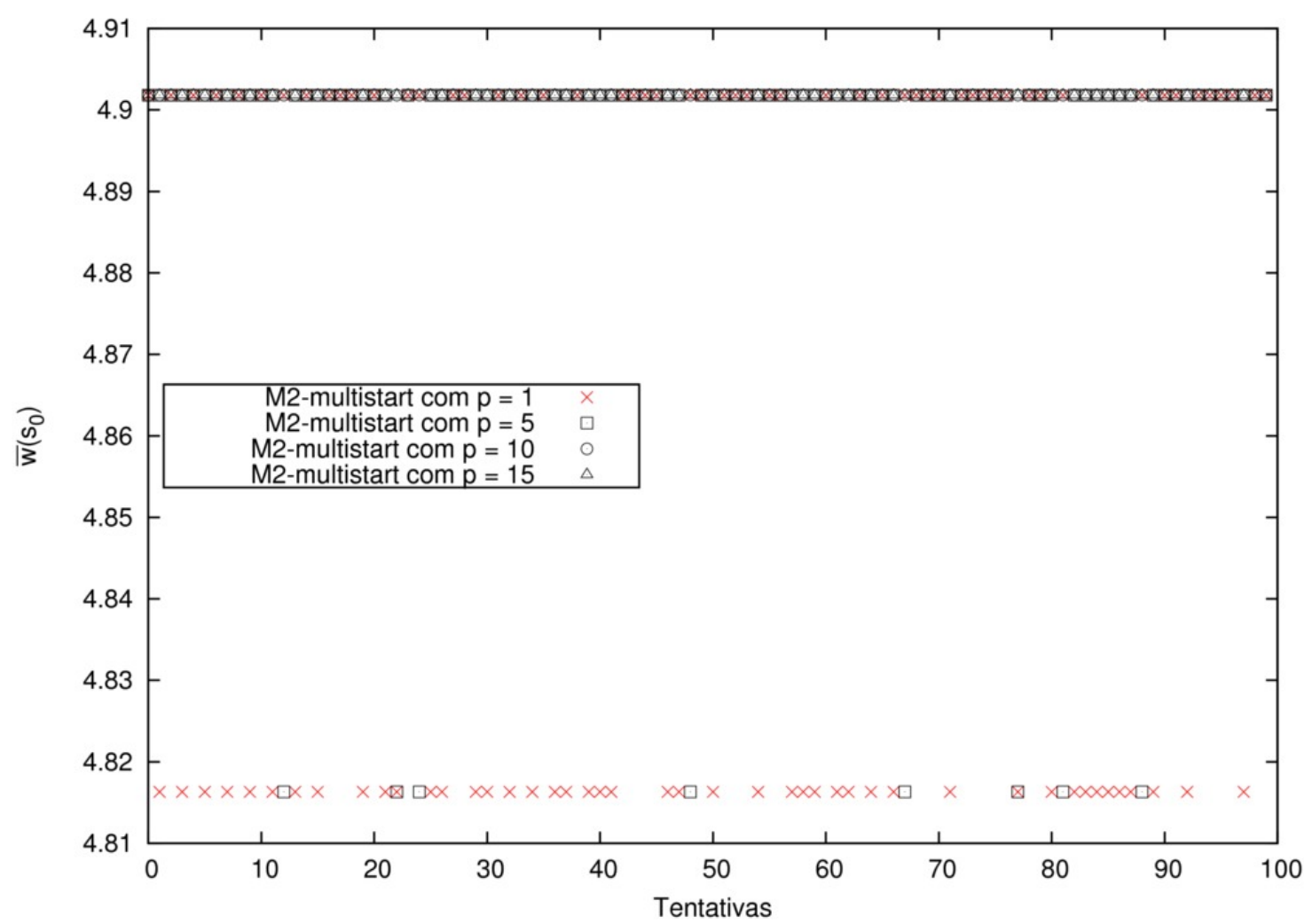

(b) Problema com $x_{0}=x_{0}^{\prime \prime}$.

Figura 4.3: $\operatorname{Em}(a)$ e (b) foi utilizado um método de multistart a fim de evitar que o método obtenha um máximo local ao invés de um global. Para as condições iniciais $x_{0}^{\prime}$ e $x_{0}^{\prime \prime}$ foram realizadas 100 tentativas de resolver o problema. Para cada tentativa o SPG foi executado a partir de $p=1,5,10$ e 15 a partir de um ponto aleatório dentro da bola de raio $\delta$ retendo a melhor solução. 


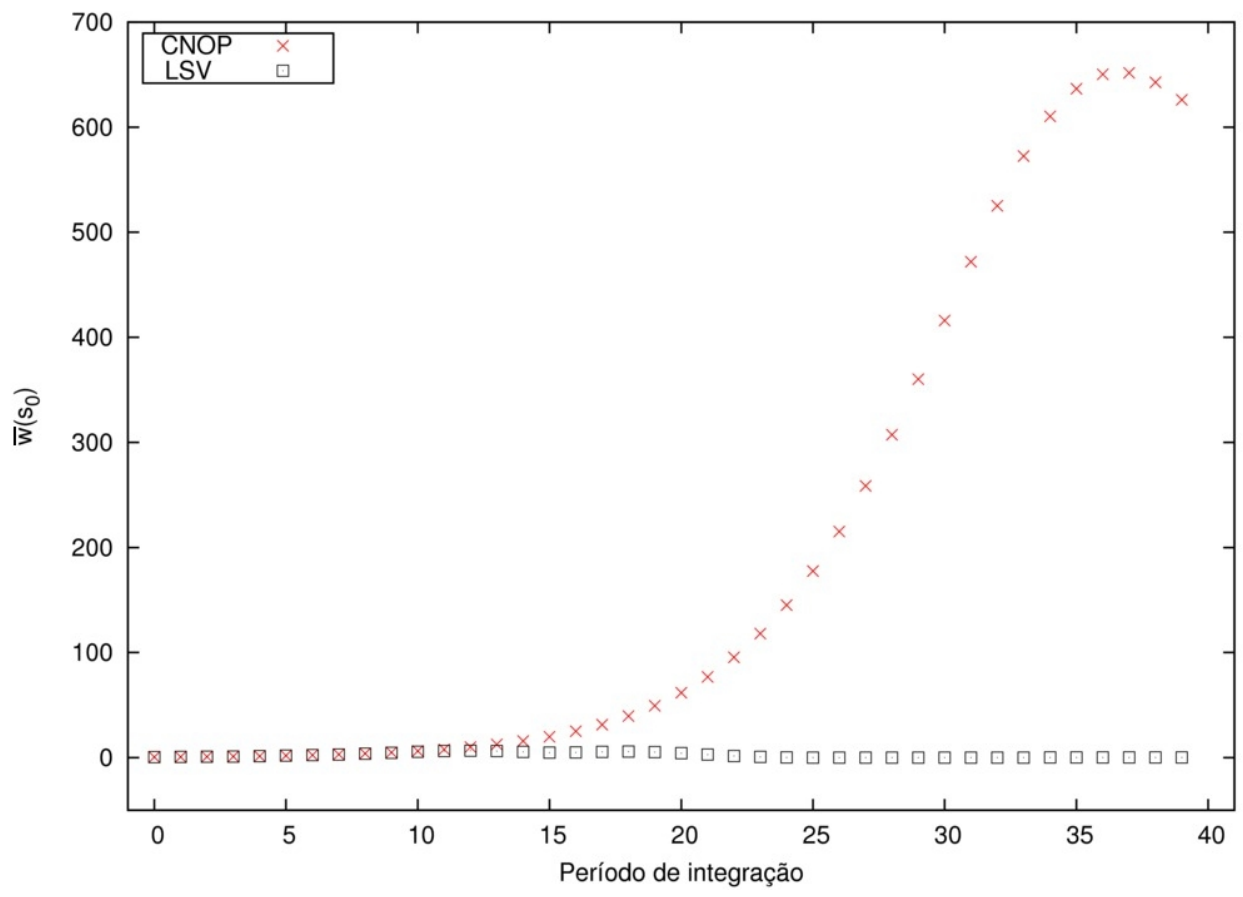

(a) Períodos de $1 \Delta t$ até $40 \Delta t$ a partir da condição inicial $x_{0}^{\prime}$

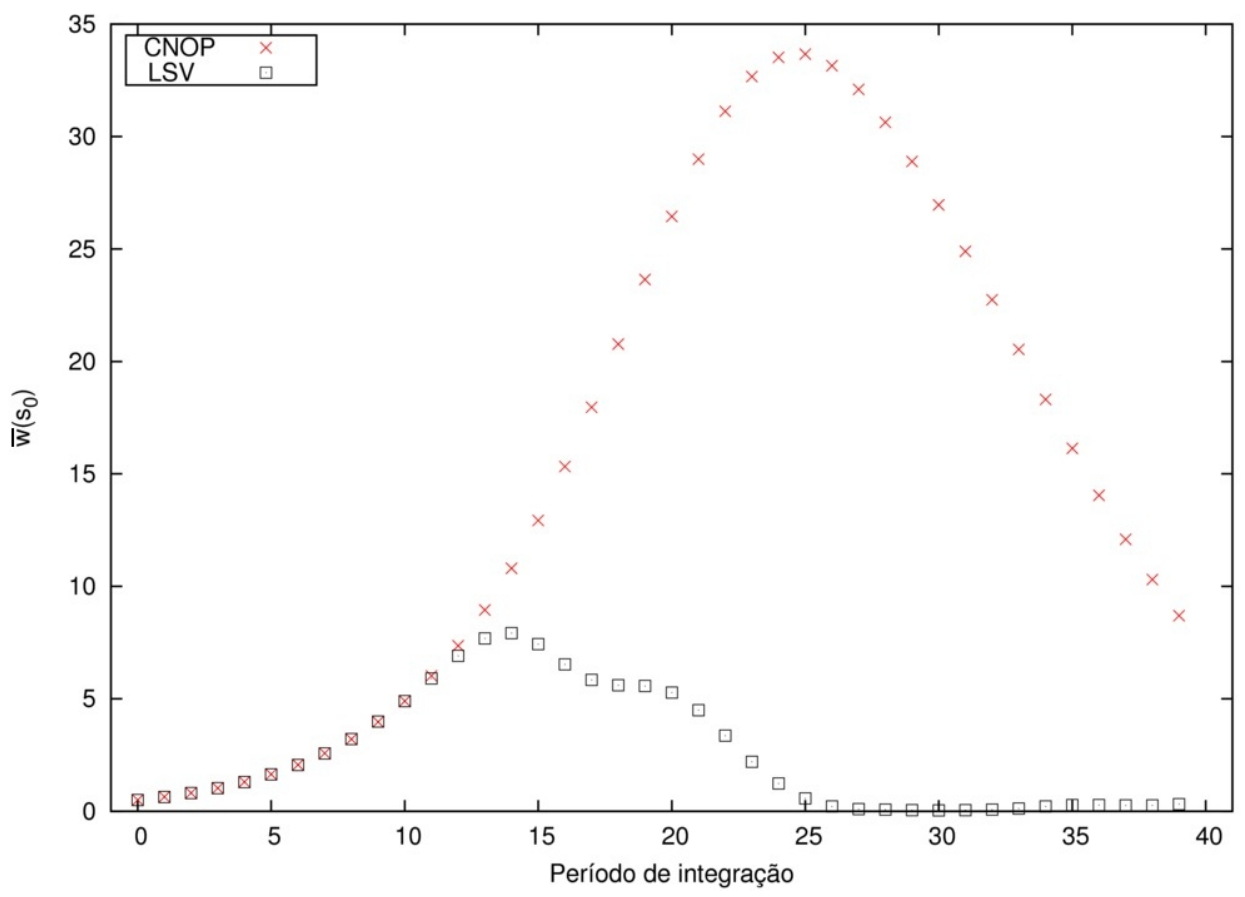

(b) Períodos de $1 \Delta t$ até $40 \Delta t$ a partir da condição inicial $x_{0}^{\prime \prime}$

Figura 4.4: $\operatorname{Em}$ (a), para a condição inicial $x_{0}^{\prime}$, com $\Delta t=0.01$ e $\delta=1.0$ são mostrados os valores da função objetivo (3.19) calculados a partir das CNOPs e dos LSVs para o sistema de Lorenz. O mesmo foi calculado em (b) para o ponto $x_{0}^{\prime \prime}$. 


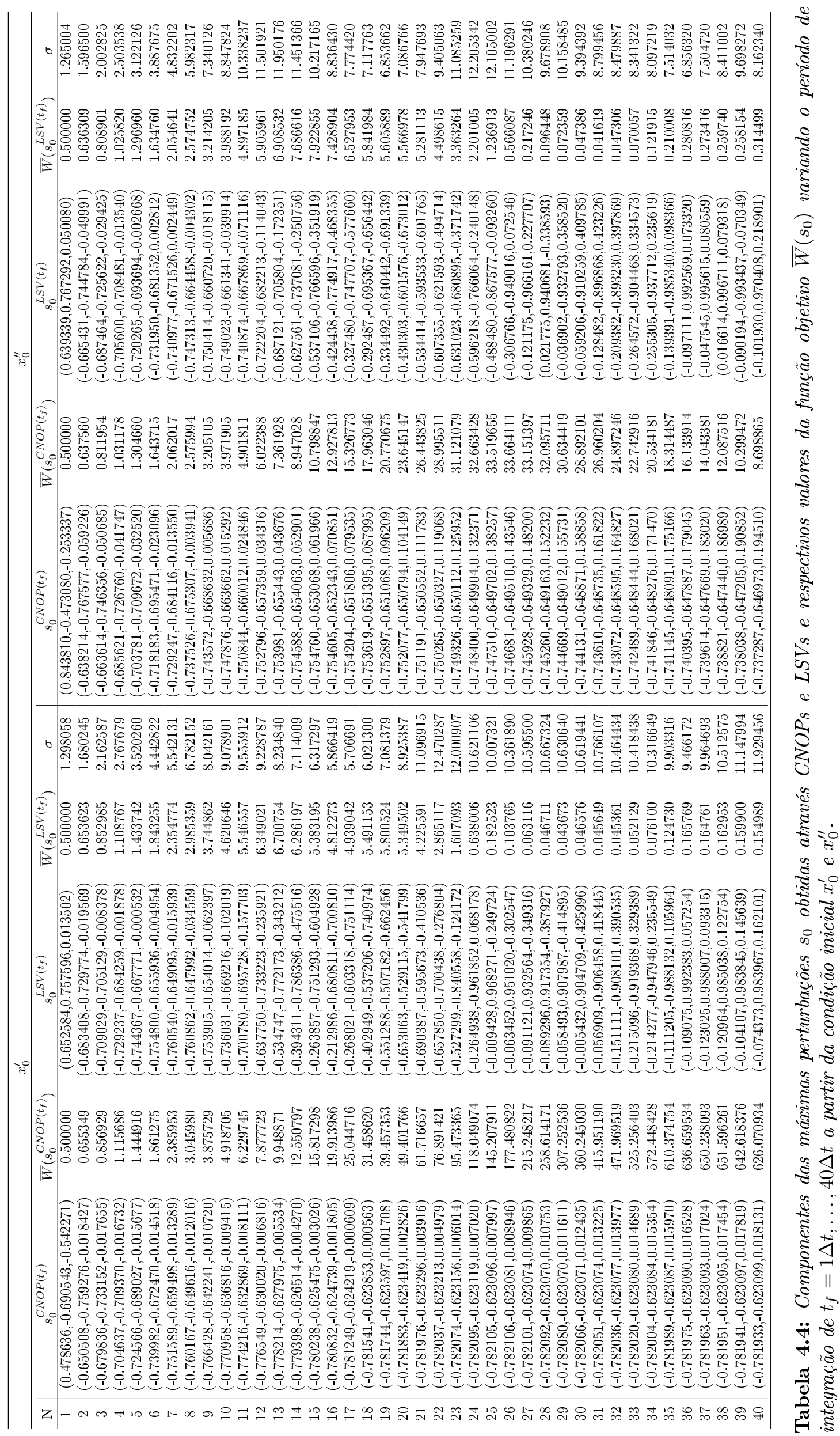




\section{Capítulo 5}

\section{Aplicações em previsibilidade, estabilidade e sensibilidade}

Neste capítulo estudamos os três tipos de problemas abordados na Meteorologia onde as perturbações condicionais não-lineares ótimas são empregadas, a saber, problemas de previsibilidade, estabilidade e sensibilidade.

\subsection{Previsibilidade}

Dando início ao estudo dos problemas em que o SPG é empregado em Meteorologia, nesta seção abordamos a questão da previsibilidade de sistemas através das CNOPs, conforme mostrado em [31] (pg.22). O estudo baseia-se no modelo de Lorenz de 1963 [29] abordado no Capítulo 4.

Segundo [30], uma característica interessante do modelo está associada ao fato de que suas propriedades de previsibilidade locais variam substancialmente com a posição relativa ao atrator. Regiões do espaço de fase onde a previsibilidade é pequena são distintas de regiões onde o fluxo é quaseestacionário.

A teoria estudada nos capítulos anteriores pode ser aplicada na estimativa das perturbações lineares que possuem o maior crescimento sobre uma porção finita da trajetória de um sistema. Neste caso, estas perturbações são a expressão da previsibilidade em cada ponto do espaço de fase, e este é o tema que aplicaremos ao modelo de Lorenz de 1963 estudado a seguir.

O modelo de Lorenz de 1963 [29], cujas características foram brevemente comentadas no Capítulo 4, em sua forma mais popular (vide [29, 30, 31, 32, 33, 34, 35]), é descrito por

$$
\left(\begin{array}{c}
\frac{d x_{1}}{d t} \\
\frac{d x_{2}}{d t} \\
\frac{d x_{3}}{d t}
\end{array}\right)=\left(\begin{array}{ccc}
-\sigma & \sigma & 0 \\
r & -1 & -x_{1} \\
x_{2} & 0 & -b
\end{array}\right)\left(\begin{array}{l}
x_{1} \\
x_{2} \\
x_{3}
\end{array}\right) .
$$

Inicialmente obtivemos as evoluções temporais e o retrato de fase para o sistema de Lorenz com $\sigma=10, b=8 / 3, r=28$ a partir da condição inicial $x_{0}=(1.0,1.0,1.0)^{T}$. O modelo foi integrado considerando $N=10000$ passos com $\Delta t=0.01$. Os resultados obtidos são mostrados nas Figuras 5.1 e 5.2 .

De acordo com [35], a forma geral das Figuras 5.2(a-d) não depende da condição inicial escolhida nem mesmo do método numérico empregado. Os detalhes, entretanto, dependem fortemente destes fatores. A sequência exata de voltas da trajetória é extremamente sensível a escolhas da condição inicial e muda com o método de integração, o que torna impossível prever como a trajetória evolui em qualquer período que não seja curto. De maneira geral os resultados obtidos no presente trabalho são compatíveis com os apresentados na literatura [35].

A partir dos resultados da Figura 5.2 nos propusemos a calcular a previsibilidade de cada ponto da trajetória. Para cada estado do sistema obtivemos uma estimativa para o máximo erro de previsão 


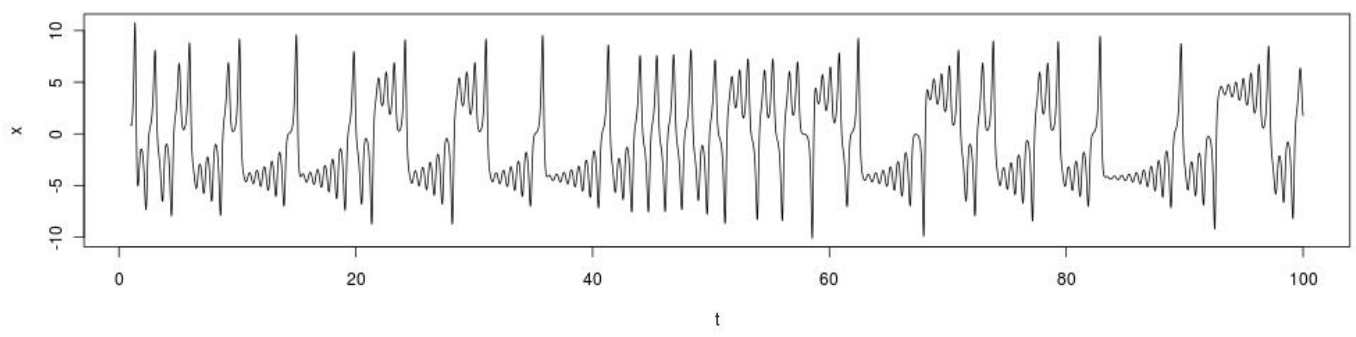

(a) Evolução Temporal de $\mathrm{x}$.

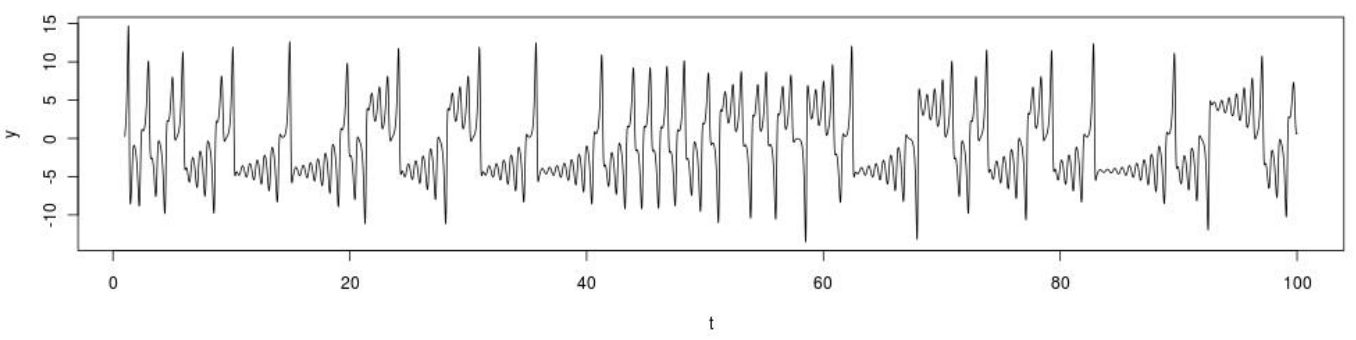

(b) Evolução Temporal de y.

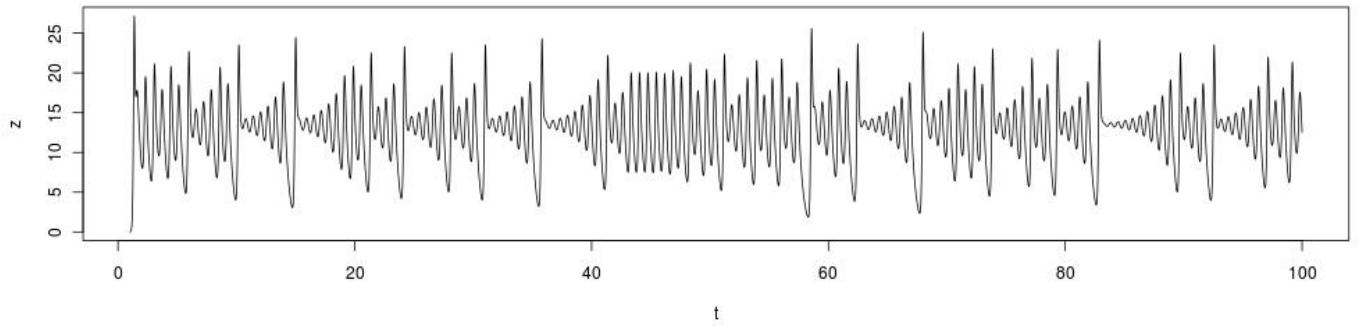

(c) Evolução Temporal de z.

Figura 5.1: Evolução no tempo de cada uma das variáveis de estado do sistema.

a partir de uma dada condição inicial utilizando as estratégias de LSVs e CNOPs e para horizontes de tempo dados por $\Delta t=0.01$ e $N \in\{10,30,100\}$.

Resolver o problema proposto a partir dos LSVs foi descrito na Seção 3.2 a partir da solução do problema (3.11). Em nossa implementação, o cálculo requerido de vetores e valores singulares foi realizado utilizando a rotina gsl_linalg_SV_decomp da biblioteca GSL. Abordar o problema de previsibilidade através das CNOPs, de acordo com o descrito na Seção 3.3, consiste em resolver o problema de programação matemática (3.19), através da abordagem Multistart-SPG.

A análise de previsibilidade reside na ideia de que os pontos onde o valor singular dominante é maior são pontos onde os erros nas condições iniciais possuem maior impacto na evolução do sistema, e portanto, são menos previsíveis. Os resultados dos dois experimentos realizados são mostrados nas Figuras 5.3(a-f). Na Figura 5.3(a) observa-se que para $N=10$ existe uma região mais suscetível a erros próxima da origem. Esta região se amplia no caso $N=30$ como pode ser observado na Figura 5.3(c) e caminha para regiões superiores do atrator para o caso $N=100$ como mostra a Figura 5.3(e). Ao analisar as figuras correspondentes utilizando CNOPs (Figuras 5.3(b,d,f)) observase que nos três casos as figuras obtidas a partir de LSVs subestimam os indicadores de sensibilidade dos pontos. Além disso, a análise utilizando CNOPs revela pontos sensíveis em regiões mais amplas 


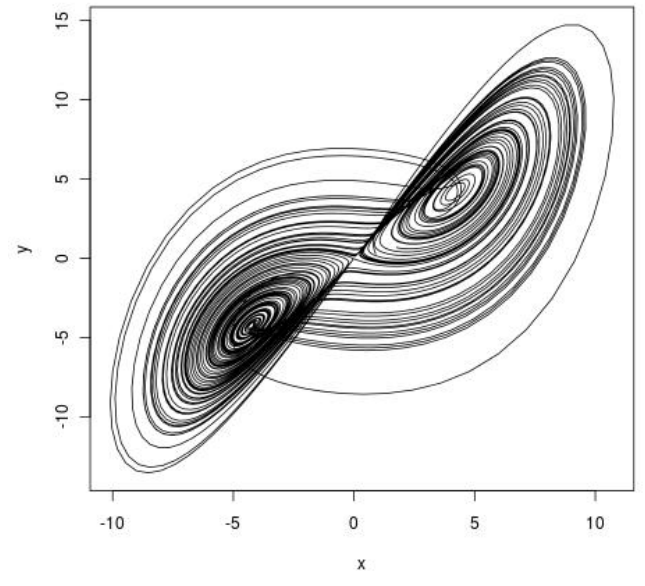

(a) Plano xy.

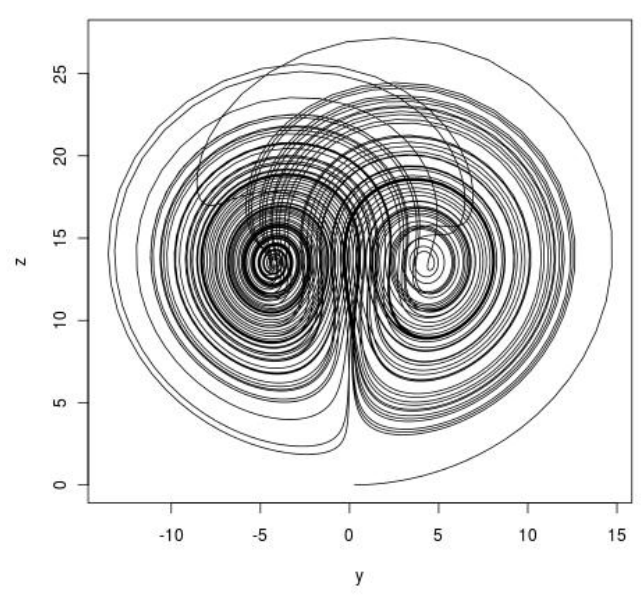

(c) Plano yz.

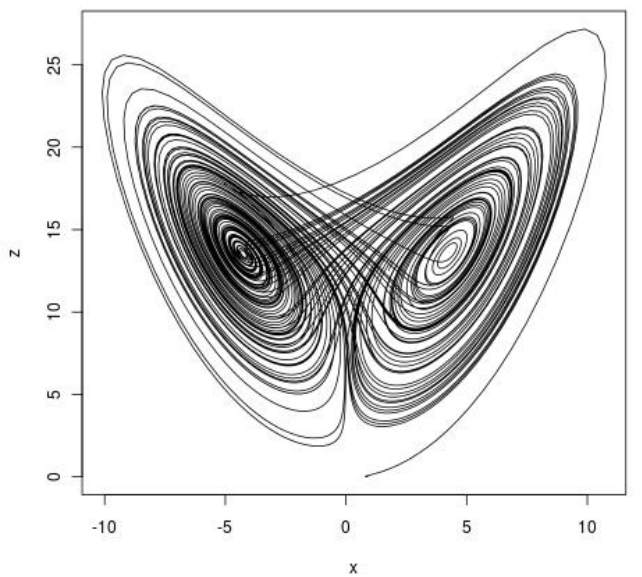

(b) Plano xz.

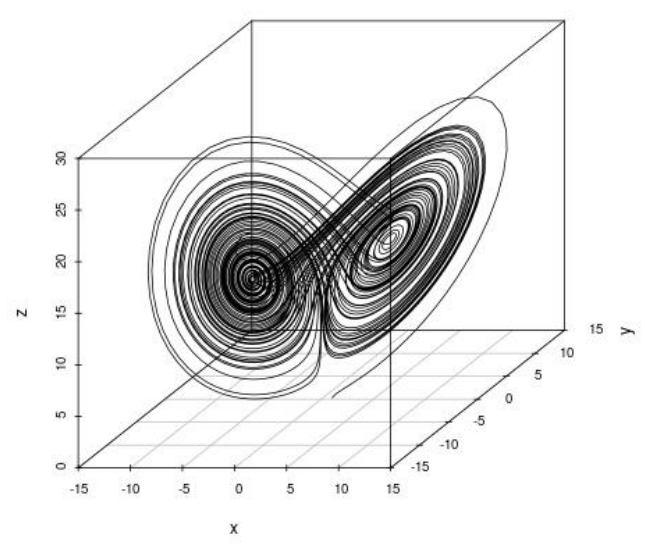

(d) Retrato de Fase 3D.

Figura 5.2: A projeções do retrato de fase do sistema (5.1) também conhecido como atrator de Lorenz. Em (a) a projeção sobre o eixo $x y$, em (b) a projeção sobre xz e em (c) a projeção sobre yz. Temos também o retrato de fase tridimensional (d).

para períodos de simulação mais longos que não eram revelados pelo método dos LSVs. A título de ilustração, apresentamos as coordenadas de cada ponto da trajetória e os valores das perturbações que maximizaram a função objetivo (3.15) para LSVs e CNOPs na Tabela 5.1.

Além disto outras noções podem ser discutidas a partir destes resultados. Segundo [31] a análise realizada anteriormente não é apenas relevante na descrição da resposta de sistemas a erros iniciais mas também na descrição da resposta de sistemas a alguma perturbação externa, onde cita-se como exemplo o estudo de variações paleoclimáticas na insolação sobre a Terra devido ao fenômeno de precessão dos equinócios.

Segundo [31], enquanto a média anual da variação da insolação (devido a estas variações orbitais) é próxima de zero perto do equador (e mesmo a média sobre toda a Terra), a resposta média anual do sistema sob efeito das perturbações não é zero. Conforme podemos observar na Figura 5.3, o sistema de Lorenz é mais sensível a perturbações impostas nas regiões onde o valor singular é maior. Disto observa-se que a resposta do sistema a uma forçante com uma média zero no tempo não necessariamente é zero uma vez que depende da sensibilidade do ponto onde é aplicada. Consideremos 


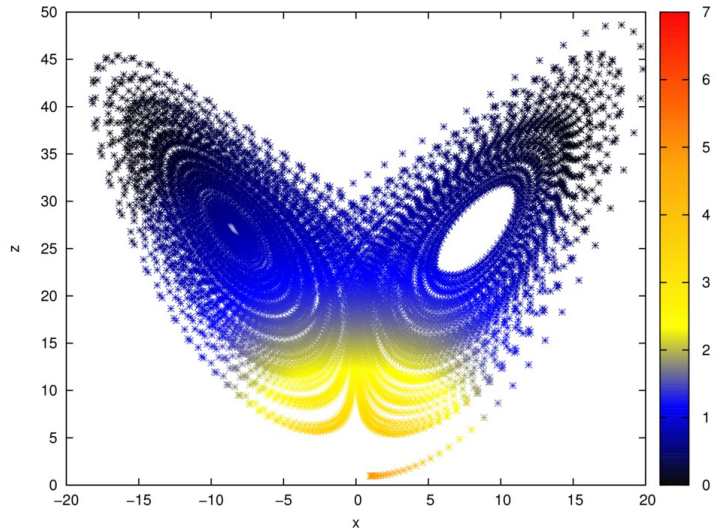

(a) Previsibilidade estimada utilizando LSVs para o problema com $N=10$.

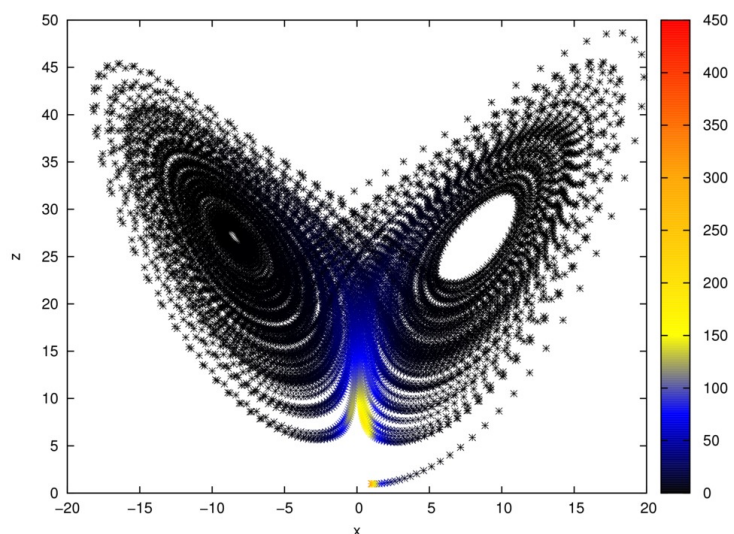

(c) Previsibilidade estimada utilizando LSVs para o problema com $N=30$.

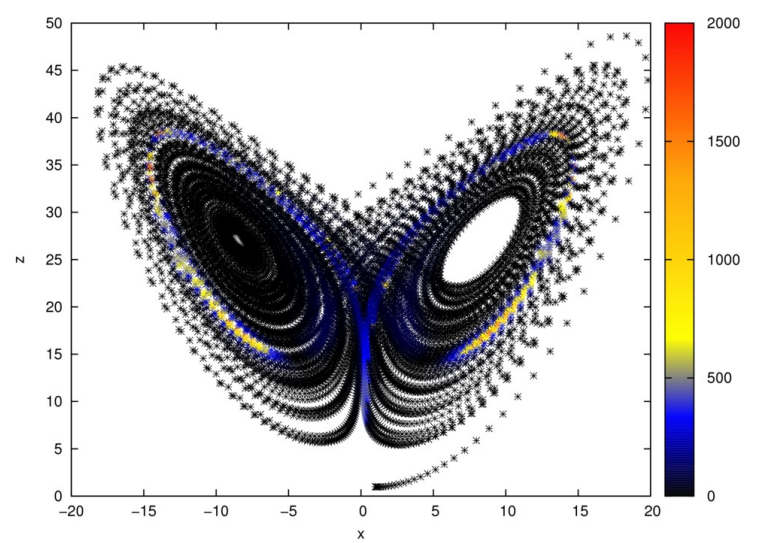

(e) Previsibilidade estimada utilizando LSVs para o problema com $N=100$.

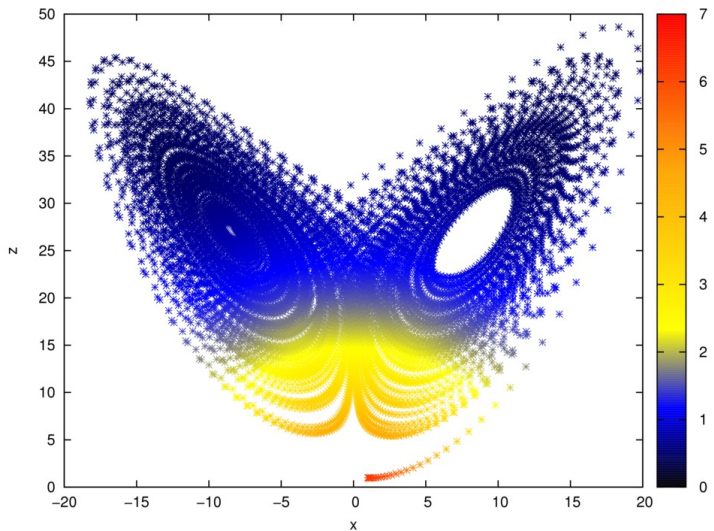

(b) Previsibilidade estimada utilizando CNOPs para o problema com $N=10$.

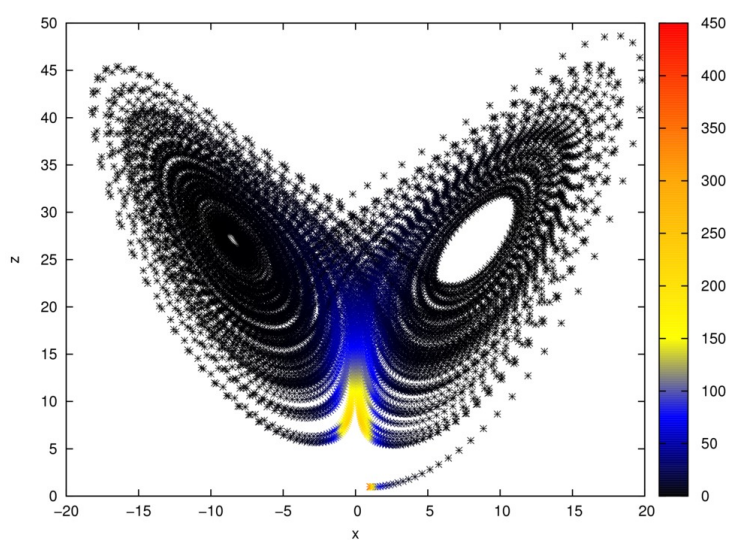

(d) Previsibilidade estimada utilizando CNOPs para o problema com $N=30$.

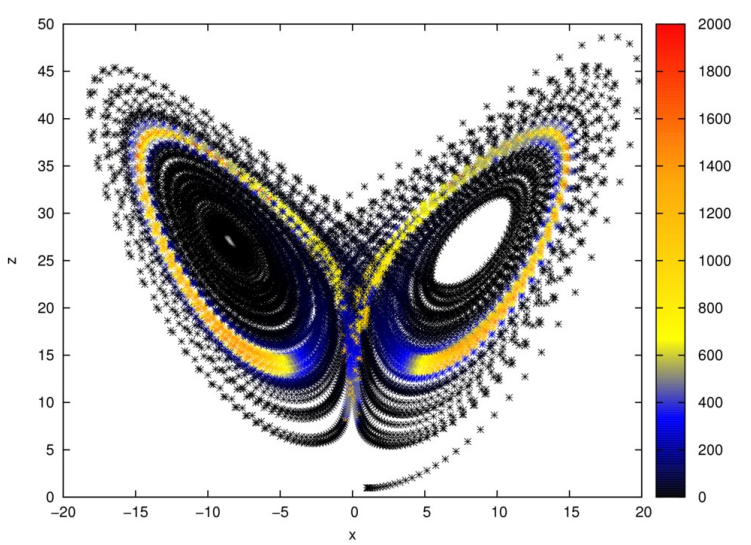

(f) Previsibilidade estimada utilizando CNOPs para o problema com $N=100$.

Figura 5.3: Os gráficos $(a, c, e)$ e $(b, d, f)$ estão relacionados às estimativas da perturbação de máximo crescimento calculadas utilizando LSVs e CNOPs, respectivamente. Foi considerado um periodo de integração de $N \delta t \operatorname{com} \delta t=0.01$ e $N=10$ nos gráficos (a) e (b), $N=50$ nos gráficos (c) e (d) e $N=100$ nos gráficos (e) $e(f)$. Em todos as figuras, a cor representa a norma da evolução da perturbação calculada.

por exemplo, uma força $f=\varepsilon(\bar{Z}-Z)$, onde $\varepsilon$ é uma constante positiva e $\bar{Z}$ é a média temporal da variável $Z$ no sistema de Lorenz não perturbado. Quando $Z \approx 0$ o vetor de estados está numa região relativamente sensível e uma perturbação negativa qualquer terá um efeito relativo. Por outro lado, quando $Z>\bar{Z}$, o vetor de estados está numa região de baixa sensibilidade e a forçante tem pouco 
ou nenhum efeito. Finalmente, a aplicação das CNOPs ao problema revela que alguns pontos do sistema tem previsibilidade menor do que o tradicionalmente calculado por LSVs além de existirem outras regiões no espaço de estados do sistema em que existem pontos sensíveis a perturbações além dos previstos tradicionalmente.

\subsection{Estabilidade}

Nesta seção abordaremos um problema de estabilidade no contexto do modelo teórico de ecossistema descrito em [19]. O modelo é utilizado no estudo das interações entre biosfera-atmosfera e das transições no tempo entre ecossistemas de áreas semi-áridas. Uma vez que ecossistemas só são possíveis em condições de equilíbrio, coexistem sob condições climáticas favoráveis e respondem a mudanças das condições climáticas, estudos sobre a estabilidade de tais sistemas a perturbações de origem humana ou climática encontram nos métodos aqui estudados uma ferramenta de grande valor.

Para estudar o problema de encontrar a perturbação que tem o maior impacto no equilíbrio de um sistema, reproduzimos o trabalho [38], baseado no modelo prognóstico simplificado com duas variáveis de um ecossistema de pradaria [39, 40].

Dado $x=\left(x_{1}, x_{2}, x_{3}\right)^{T}$ consideraremos o modelo utilizado nos trabalhos [19, 38] descrito por

$$
\left(\begin{array}{c}
a \frac{d x_{1}}{d t} \\
b \frac{d x_{2}}{d t} \\
c \frac{d x_{3}}{d t}
\end{array}\right)=\left(\begin{array}{c}
F_{1}(x) \\
F_{2}(x) \\
F_{3}(x)
\end{array}\right)=\left(\begin{array}{c}
G\left(x_{1}, x_{2}\right)-D\left(x_{1}, x_{2}\right)-C\left(x_{1}\right) \\
P-E_{s}\left(x_{1}, x_{2}, x_{3}\right)-E_{r}\left(x_{1}, x_{2}\right)-R\left(x_{1}, x_{2}, x_{3}\right) \\
G_{z}\left(x_{1}, x_{2}\right)-D_{z}\left(x_{3}\right)-C\left(x_{1}, x_{2}, x_{3}\right)
\end{array}\right) .
$$

O modelo (5.2) consiste num modelo não-linear de ecossistema que considera uma única coluna vertical de solo, uma espécie de grama e possui três variáveis de estado adimensionais. $x_{1}$ é a densidade de massa de grama viva, $x_{2}$ a umidade disponível no solo e $x_{3}$ a densidade de massa de grama murcha. Temos ainda que $a, b$ e $c$ são constantes adimensionais correspondentes aos valores característicos do modelo. As funções $G\left(x_{1}, x_{2}\right), D\left(x_{1}, x_{2}\right)$ e $C\left(x_{1}\right)$ representam o crescimento através de sintetização de massa por fotossíntese, murchamento e consumo das folhas vivas (através de pastoreio), $P$ é a precipitação atmosférica (entrada do sistema). $E_{s}\left(x_{1}, x_{2}, x_{3}\right)$ é a evaporação da superfície do solo, $E_{r}\left(x_{1}, x_{2}\right)$ a transpiração e $R\left(x_{1}, x_{2}, x_{3}\right)$ é o runoff (água de precipitação não absorvida pelo solo). $G_{z}\left(x_{1}, x_{2}\right), D_{z}\left(x_{3}\right)$ e $C_{z}\left(x_{1}, x_{2}, x_{3}\right)$ são a acumulação, decomposição e consumo da grama murcha, respectivamente.

As expressões das funções que compõem o modelo tem a forma:

$$
\begin{aligned}
G\left(x_{1}, x_{2}\right) & =\alpha^{*}\left(1-e^{-\epsilon_{g} x_{1}}\right)\left(1-e^{-\epsilon_{g} x_{2}}\right), \\
D\left(x_{1}, x_{2}\right) & =\alpha^{*} \beta\left(e^{\epsilon_{d} x_{1}}-1\right)\left(1-e^{-\epsilon_{d}^{\prime} x_{2}}\right)^{-1}, \\
C\left(x_{1}\right) & =\alpha^{*} \gamma\left(1-e^{-\epsilon_{c} x_{1}}\right), \\
E_{s}\left(x_{1}, x_{2}, x_{3}\right) & =e^{*}\left(1-e^{-\epsilon_{2} x_{2}}\right) e^{-\epsilon_{3} x_{3}}\left(\left(1-\sigma_{f}\right)+\sigma_{f}\left(1-\kappa_{1}\left(1-e^{-\epsilon_{1} x_{1}}\right)\right)\right), \\
E_{r}\left(x_{1}, x_{2}\right) & =e^{*} \phi_{r s}\left(1-e^{-\epsilon_{2}^{\prime} x_{2}}\right) \sigma_{f}\left(1-\kappa_{1}^{\prime} e^{-\epsilon_{1}^{\prime} x_{1}}\right), \\
R\left(x_{1}, x_{2}, x_{3}\right) & =\lambda e^{*} \mu\left(e^{\epsilon_{3}^{\prime \prime} x_{2}}-1\right) e^{\epsilon_{3}^{\prime \prime} x_{3}}\left(\left(1-\sigma_{f}\right)+\sigma_{f}\left(1-\kappa_{1}^{\prime \prime}\left(1-e^{\epsilon_{1}^{\prime \prime}}\right)\right)\right), \\
G_{z}\left(x_{1}, x_{2}\right) & =\alpha^{*} \alpha_{z} \beta\left(e^{\epsilon_{d} x_{1}}-1\right)\left(1-e^{-\epsilon_{d}^{\prime} x_{2}}\right)^{-1}, \\
D_{z}\left(x_{3}\right) & =\alpha^{*} \beta_{z}\left(e^{\epsilon_{d_{3}} x_{3}}-1\right), \\
C_{z}\left(x_{3}\right) & =\alpha^{*} \gamma_{z}\left(1-e^{-\epsilon_{c_{3}} x_{3}}\right) .
\end{aligned}
$$

Nosso objetivo aqui é calcular as CNOPs reproduzindo os resultados obtidos em [19] (pg.413). Para tanto, implementamos o modelo (5.2) composto pelas equações (5.3) utilizando os parâmetros fornecidos nas Tabelas 5.2 e 5.3 obtidos de $[39,40]$ (outros valores para a constante $\mu$ também foram 


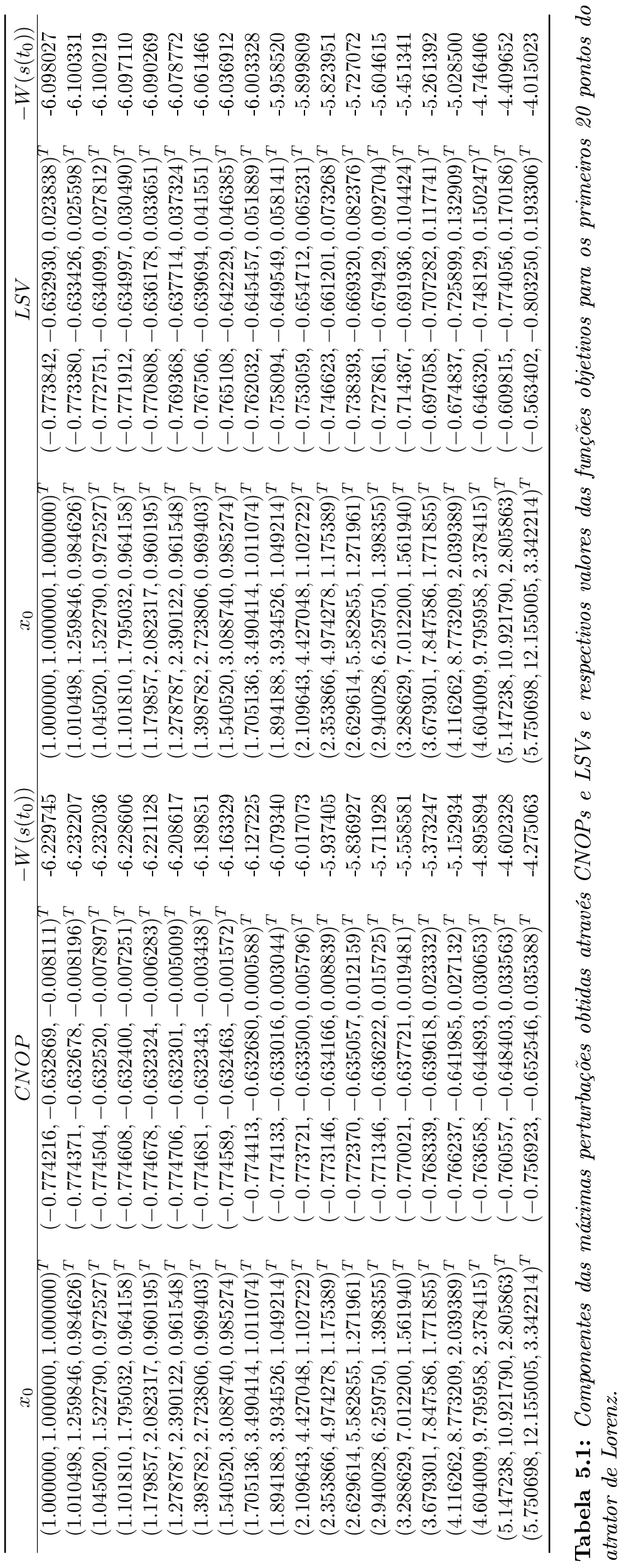


considerados em [19]).

Um ponto $x^{*}=\left(x_{1}^{*}, x_{2}^{*}, x_{3}^{*}\right)^{T}$ no contexto do sistema (5.2) é um ponto de equilíbrio se satisfaz a relação $F_{1}\left(x^{*}\right)=F_{2}\left(x^{*}\right)=F_{3}\left(x^{*}\right)=0$. Segundo [19], para um parâmetro $\mu=0.32$ existem dois pontos de equilíbrio $x^{*}$ distintos denominados GES (estado de equilíbrio de estepe, do inglês Grassland Equilibrium State) e DES (estado de equilíbrio desértico, do inglês Desert Equilibrium State). Em $x_{G E S}^{*}$ o ecossistema é estável numa condição de estepe e em $x_{D E S}^{*}$ o ecossistema apresenta-se numa condição desértica.

\begin{tabular}{lc}
\hline Parâmetros dimensionais \\
\hline$a$ & $0.1 \mathrm{Kg} \mathrm{m}^{-1}$ \\
$b$ & $240 \mathrm{~mm}$ \\
$c$ & $0.1 \mathrm{Kg} \mathrm{m}^{-2}$ \\
$\alpha$ & $0.4 \mathrm{Kg} \mathrm{m}^{-2}$ ano $^{-1}$ \\
\hline
\end{tabular}

Tabela 5.2: Coeficientes dimensionais do modelo.

\begin{tabular}{cc}
\hline Coeficientes adimensionais \\
\hline$\mu$ & 0.32 \\
$\beta, \beta_{z}, \gamma$ & 0.1 \\
$\gamma_{z}$ & 0 \\
$\alpha_{z}$ & 0.5 \\
$\sigma_{f}, \epsilon_{g}, \epsilon_{g}^{\prime}, \epsilon_{d}, \epsilon_{d}^{\prime}, \epsilon_{c}$ & 1.0 \\
$\epsilon_{d z}$ & 1.0 \\
$\phi_{r s}$ & 0.7 \\
$\lambda$ & 0.015 \\
$\kappa_{1}$ & 0.3 \\
$\kappa_{1}^{\prime \prime}$ & 0.4 \\
$\kappa_{1}^{\prime}$ & 1.0 \\
$\epsilon_{1}, \epsilon_{1}^{\prime \prime}$ & 0.7 \\
$\epsilon_{1}, \epsilon_{2}, \epsilon_{2}^{\prime}, \epsilon_{2}^{\prime \prime}, \epsilon_{3}, \epsilon_{3}^{\prime \prime}$ & 1.0 \\
\hline
\end{tabular}

Tabela 5.3: Coeficientes adimensionais do modelo.

Os valores das condições de equilíbrio iniciais são os estados do sistema no tempo $t_{f}=10$. Para tanto implementamos o código apresentado no Apêndice 6, e obtivemos $x_{D E S}^{*}$ e $x_{G E S}^{*}$ utilizando Runge-Kutta de ordem 4 com um passo $\Delta t=0.1$ para $N=100$ passos. Cada $\Delta t=0.1$ representa o período de 0.1 ano. Para o equilíbrio GES obtivemos $x_{G E S}^{*}=(0.641690,0.649032,0.652603)^{T}$, e para o equilíbrio DES obtivemos $x_{D E S}^{*}=(0.0,0.382380,0.0)^{T}$, ambos compatíveis com os pontos obtidos em [19].

A partir destes dois pontos de equilíbrio calculamos as CNOPs para diferentes valores de $\delta$. Para analisar a estabilidade de $x_{G E S}^{*}$ calculamos CNOPs com $\delta \in\{0.2,0.4,0.5,0.6\}$, e para o $x_{D E S}^{*}$ variamos $\delta \in\{0.2,0.3,0.5\}$. Em ambos os casos o tempo total de integração foi $t_{f}=10$ anos, com $N=100$ passos, ou seja, um passo de integração de tamanho $\Delta t=0.1$ ano. Utilizamos o método Multistart-SPG conforme apresentado na Seção 4.2. Os resultados obtidos são apresentados na Tabela 5.4, e são muito próximos dos resultados apresentados em [19].

Dando continuidade ao estudo de [19], apresentamos as Figuras 5.4 e 5.5, afim de investigar a evolução não-linear do modelo (5.2) para $x(t)=x^{*}\left(t_{0}\right)+\hat{s}\left(t_{0}\right)$. Nas Figuras 5.4(a-c), as três componentes da evolução não-linear do ecossistema são representadas por curvas, assim como o ponto inicial $x^{*}\left(t_{0}\right)=x_{G E S}^{*}$. Nos casos $\delta=0.2,0.4$ e 0.5 , as CNOPs levam o sistema a retornar ao equilíbrio $x_{G E S}^{*}$. As três variáveis recuperam rapidamente a condição de equilíbrio, porém demorando mais tempo quanto maior é a amplitude da perturbação. Com $\delta=0.2, x_{1}$ retoma o equilíbrio em $t \approx 30$ 


\begin{tabular}{cc|cc}
\hline$\delta$ & CNOPs para $x_{G E S}^{*}$ & $\delta$ & CNOPs para $x_{D E S}^{*}$ \\
\hline 0.2 & $(-0.112551,-0.085014,-0.141791)^{T}$ & 0.2 & $(0.167284,0.043360,0.100678)^{T}$ \\
0.4 & $(-0.260973,-0.148529,-0.264258)^{T}$ & 0.3 & $(0.231971,0.080387,0.172416)^{T}$ \\
0.5 & $(-0.372209,-0.159673,-0.293198)^{T}$ & 0.5 & $(0.340270,0.167041,0.326058)^{T}$ \\
0.6 & $(-0.585272,-0.063326,-0.115960)^{T}$ & - & - \\
\hline
\end{tabular}

Tabela 5.4: CNOPs calculadas para os pontos $x_{G E S}^{*}$ e $x_{D E S}^{*}$ para um período de 10 anos.

anos. Com $\delta=0.4, x_{1}$ retoma o equilíbrio em $t \approx 50$ anos enquanto com $\delta=0.5, x_{1}$ retoma o equilíbrio em $t \approx 80$ anos. Também verifica-se que uma perturbação $\delta=0.6$ conduz o sistema a uma condição de equilíbrio GES.

Analisando o exemplo de $\delta=0.6$, de acordo com [19], verifica-se que $d x_{1} / d t$ e $d x_{2} / d t$ são positivos e $d x_{3} / d t$ é negativo no início. A densidade de massa de grama viva $x_{1}$ aumenta enquanto a densidade de grama morta diminui, e por um curto período de tempo a umidade do solo $x_{2}$ aumenta. Observa-se o crescimento de $x_{1}$ por aproximadamente cinco anos e o decréscimo rápido de $x_{3}$ pelo mesmo período causando um aumento do consumo e evaporação da água do solo. Isto explica o rápido crescimento de $x_{2}$ por aproximadamente 3 anos e seu posterior decaimento rápido. Ao final temos a grama perdendo suas condições de sobrevivência e viabilidade. Depois de crescer por aproximadamente 5 anos, $x_{1}$ decai e o ecossistema de pradaria torna-se um deserto do tipo DES.

Para a situação DES mostrada nas Figuras 5.5(a-c) observa-se que nos casos $\delta=0.2$ e 0.3 , o ecossistema perturbado é atraído de volta para a condição DES. As três variáveis recuperam sua condição inicial num curto período de tempo após serem perturbadas, embora uma perturbação maior faça com que o sistema leve mais tempo para se recuperar. Para $\delta=0.5$, o ecossistema evolui para uma condição GES. Observa-se que os valores que satisfazem $d x_{1} / d t$ e $d x_{2} / d t$ são negativos e $d x_{3} / d t$ são positivos. Assim, num primeiro momento a densidade de massa da grama viva $x_{1}$ e a umidade do solo $x_{2}$ decrescem e a densidade de massa da grama morta $x_{3}$ aumenta. Um aumento em $x_{3}$ causa uma redução da evaporação da umidade do solo além do decréscimo em $x_{1}$ causar a redução do consumo de água. Portanto, depois de decrescer por aproximadamente 1 ano $x_{2}$ aumenta de repente. Por outro lado, o valor inicial de $x_{1}$ é grande e depois de decrescer por 3 anos, $x_{1}$ aumenta e o ecossistema evolui para um estado de pradaria ao final.

\subsection{Sensibilidade}

Nesta sessão continuamos nosso estudo das aplicações do SPG em Meteorologia. Abordamos um problema de sensibilidade conforme apresentado em [41, 42]. Neste caso, o objetivo é determinar o ponto do sistema mais sensível a perturbações nas observações. Com isto espera-se minimizar o erro médio das previsões para um determinado período ao se realizar uma observação adicional neste ponto específico. A literatura descreve este problema como um problema de targeting.

O modelo teórico de Lorenz de 1996 é descrito em [43]. Conforme comentado pelo autor, o modelo leva em consideração as principais características da atmosfera real em sua descrição, e tem a forma

$$
\frac{d p_{j}}{d t}=\left(p_{j+1}-p_{j-2}\right) p_{j-1}-p_{j}+F, j=1, \ldots, J,
$$

onde $J$ é a dimensão do sistema e $F$ é uma constante. Para que o sistema esteja bem definido para todos os valores de $j$ estabelece-se que $p_{-1}=p_{J-1}, p_{0}=p_{J}$ e $p_{J+1}=p_{1}$. Desta forma, a componente $p_{j}$ do vetor $p \in \mathbb{R}^{J}$ pode ser vista como o valor de alguma grandeza escalar da Meteorologia (tal como a temperatura) no ponto geográfico $q_{j}$ estendendo-se ao longo de um círculo de latitudes variáveis formando uma cadeia de pontos geográficos equidistantes entre si.

Em nossos experimentos, dois métodos foram testados. Ambos baseiam-se na estratégia de escolha de pontos através do cálculo do ponto mais sensível. Considera-se como ponto geográfico mais 
sensível a perturbações àquele que corresponde à componente $j$ de módulo da perturbação de maior crescimento. A este ponto geográfico escolhe-se como alvo para realizar a observação $p_{j}$ observado com maior acurácia. Desta forma, ao aumentar a acurácia da medida em um único ponto espera-se diminuir o erro total da previsão. Em nossos experimentos obtivemos o ponto mais sensível através de LSVs conforme descrito em [41] (pg.411) e através de CNOPs.

O experimento consistiu em tomar um sistema de dimensão $J=40$ e $F=8.0$, no qual os pontos numerados de 1 até 20 estão sobre o oceano e os pontos numerados de 21 até 40 estão sobre o continente. Observações são realizadas a cada $6 \mathrm{~h}$ em todos os pontos sobre o continente mas em apenas um único ponto sobre o oceano.

O sistema de previsão e assimilação de dados que reproduzimos utiliza-se do estado "real" $y_{n j}$ do ponto $p_{j}$ no instante de tempo $t_{n}$. Afim de obter o estado inicial da série $y_{0 j}$, foram sorteados $J$ valores aleatórios de uma distribuição Gaussiana com média $F / 4$ e desvio padrão $F / 2$. O passo no tempo consistiu em $\Delta t=0.05$ equivalente a $6 \mathrm{~h}$. A partir destes valores iniciais o sistema foi integrado no período de 90 dias afim de remover comportamentos transientes e, depois destes 90 dias, por mais 5 anos. Estes valores de $y$ assim gerados serão considerados no nosso experimento como os dados reais a serem previstos. O estado $y_{360 j}$ (estado $y$ do ponto $q_{j}$ em $n=360$ ) foi utilizado como condição inicial para o processo de previsão-assimilação.

A literatura refere-se à condição inicial das previsões como "análises", denotadas por $w_{n j}$. Denotaremos por $x_{l j}^{n}$ à previsão para $y_{l j}$ calculada no instante $t_{n}\left(\operatorname{com} l>n\right.$ ) integrando o sistema de $t_{n}$ até $t_{l}$. A análise $w_{n j}$ será calculada como uma composição entre $y_{n j}$ e $x_{n j}^{n-1}$ da seguinte forma: (i) se uma medição tiver sido feita no ponto $q_{j}$ no instante $n$ temos que $w_{n j}=y_{n j}+a_{n j}$, onde $a_{n j}$ é o erro observacional de $y_{n j}$ e $y_{n j}=p j$ é o valor observado ou (ii) se não houver medição no ponto $q_{j}$ no instante $n$ então utiliza-se o valor previsto, quer dizer, $w_{n j}=x_{n j}^{n-1}$. Transcorridas $6 \mathrm{~h}$, uma nova análise $w_{n+1, j}$ é gerada e a previsão $x_{l j}^{n+1}$ é recalculada utilizando $w_{n+1, j}$ como condição inicial e integrando de $t_{n+1}$ até $t_{l}$.

Na integração para o cálculo das previsões $x_{l j}^{n}$, consideraremos $0.95 F$ no lugar de $F$ no modelo afim de simular a incapacidade de dispor de um modelo de previsão que se encaixe perfeitamente na realidade representada por $y$ através de (5.4). Assim como em [41], o erro observacional $a_{n j}$ foi gerado aleatoriamente de uma distribuição Gaussiana com média zero e desvio padrão $\varepsilon=F / 40$.

Para cada instante de tempo $t_{n}$ realiza-se uma previsão para um período de 1,3 ou 6 dias, quer dizer, calcula-se $x_{n+4, j}^{n}, x_{n+12, j}^{n}$ ou $x_{n+24, j}^{n}$. Para cada ciclo de previsão duas estratégias de targeting foram testadas, utilizando LSVs e CNOPs. A cada instante $t_{n}$ calcula-se a LSV ou a CNOP para o período desejado (1, 3 ou 6 dias) e a componente de maior módulo é utilizada para decidir que ponto $q_{j}$ (no oceano) será alvo de medição para obtenção de $p_{j}$ no instante $t_{n}$ (quer dizer, de que forma será construída a análise $w_{n}$ ). Uma terceira estratégia que escolhe $q_{j}$ (no oceano) de forma aleatória foi também utilizada como base para aferir o desempenho dos métodos.

Experimentos iniciais mostraram que a estratégia Multistart-SPG definida no Capítulo 4 não era adequada para a resolução dos subproblemas de otimização associados ao cálculo das CNOPs do presente problema, uma vez que, para períodos superiores a $20 \Delta t$, o método nem sempre obtinha o máximo global esperado. Por conta disso, alteramos os parâmetros de Multistart-SPG para maxit = 10.000 e $\varepsilon=10^{-4}$. Consideramos $p=50$ pontos aleatórios iniciais além do LSV na estratégia de multistart. Assim redefinido, Multistart-SPG recuperou a característica de sempre obter soluções que parecessem ser a solução global dos subproblemas (quer dizer, soluções sempre melhores ou iguais do que as obtidas por métodos alternativos). Para mais detalhes veja a definição do método MultistartSPG no Capítulo 4. Consideramos 40 problemas teste para o cálculo da CNOP do modelo (5.4) com $t_{0}=0, \delta t=0.05, N \in\{1, \ldots, 40\}$ e condição inicial $\left[x\left(t_{0}\right)\right]_{i}=1, i=1 \ldots, 40$. A Figura 5.6 ilustra o valor da função objetivo do problema do cálculo das CNOPs avaliada na CNOP achada pelo Multistart-SPG, na LSV e no melhor de 10000 pontos viáveis aleatórios. Como pode ser observado, o valor associado a CNOP é sempre maior ou igual do que os outros dois valores. 


\begin{tabular}{c|c|cc|cc}
\hline \multirow{2}{*}{ Experimento } & \multirow{2}{*}{ Método } & \multicolumn{4}{|c}{ Período de previsão } \\
\cline { 3 - 6 } & & \multicolumn{2}{|c}{1} & \multicolumn{2}{|c}{3} \\
\cline { 3 - 6 } A & RAND & 7.767856 & 3.875728 & 9.815784 & 8.435545 \\
& LSV & 7.201435 & 3.245149 & 10.093732 & 8.537200 \\
& CNOP & 6.172972 & 3.304665 & 9.929354 & 8.537200 \\
\hline \multirow{3}{*}{$\mathrm{B}$} & RAND & 8.054644 & 3.878969 & 10.077240 & 8.489689 \\
& LSV & 7.277595 & 3.257376 & 10.123835 & 8.413902 \\
& CNOP & 6.106684 & 3.321636 & 9.995963 & 8.526913 \\
\hline \multirow{3}{*}{$\mathrm{C}$} & RAND & 8.374210 & 3.972458 & 9.914800 & 8.513445 \\
& LSV & 7.135170 & 3.202010 & 10.019921 & 8.403541 \\
& CNOP & 6.179357 & 3.284872 & 9.955833 & 8.522867 \\
\hline \multirow{3}{*}{$\mathrm{D}$} & RAND & 7.613628 & 3.843531 & 9.736346 & 8.513073 \\
& LSV & 6.847866 & 3.171447 & 10.047881 & 8.392085 \\
& CNOP & 6.381934 & 3.400137 & 9.852477 & 8.497627 \\
\hline \multirow{2}{*}{ E } & RAND & 7.983458 & 3.942617 & 10.017906 & 8.516456 \\
& LSV & 6.847866 & 3.171447 & 10.047881 & 8.392085 \\
& CNOP & 6.381934 & 3.400137 & 9.852477 & 8.497627 \\
\hline
\end{tabular}

Tabela 5.5: Experimentos numéricos com o sistema (5.4) para 5 periodos de 5 anos utilizando CNOPs, LSVs e sorteio nas previsões para 1 e 3 dias.

Para avaliar a eficácia de cada estratégia nas previsões para 1 dia (quer dizer, 4 passos de 6h) consideramos o erro quadrático médio entre o estado real $y_{l j}$ e o estado previsto $x_{l j}^{l-4}$ para cada local $q_{j}$ ao longo de um período de 5 anos de previsões, ou seja,

$$
d_{j}^{4}=\sqrt{\frac{1}{7565-(365+4)} \sum_{n=365+4}^{7565}\left(x_{l j}^{l-4}-y_{l j}\right)^{2}}, j=1, \ldots, 40 .
$$

Analogamente, verificamos $d_{j}^{12} j=1, \ldots, 40$ para o mesmo período de 5 anos. Além disso, também verificamos $d_{j}^{4}, d_{j}^{12}$ e $d_{j}^{24}$ para um período de 90 dias de previsões. As Tabelas 5.5 e 5.6 mostram os resultados obtidos. Nas tabelas, $d_{20}^{4}, d_{20}^{12}$ e $d_{20}^{24}$ correspondem aos erros quadráticos medios no ponto $q_{20}$ associados a previsões de 1,3 e 6 dias, respectivamente, enquanto que $\bar{d}_{20-40}^{4}, \bar{d}_{20-40}^{12}$ e $\bar{d}_{20-40}^{24}$ correspondem a média dos erros quadráticos médios nos pontos continentais $q_{20}, \ldots, q_{40}$ associados a previsões de 1, 3 e 6 dias, respectivamente.

Analisando a Tabela 5.5, é possível observar que para o período de 5 anos, a comparação entre a estratégia que usa LSVs e a estratégia aleatória mostra que as LSVs apresentam erros quadráticos menores em 14 dos 20 casos considerados. Observa-se ainda que nas previsões de 1 dia, tanto para o ponto $q_{j}=20$ como sobre a média continental, houve redução do erro nas previsões. Para o período de 3 dias, LSVs não tem um bom desempenho quando comparado com o sorteio de pontos nas previsões relacionadas ao ponto $q_{20}$, mas ainda assim contribuem para reduzir o erro médio $\bar{d}_{20-40}^{12}$ das previsões sobre o continente em 4 dos 5 casos analisados. Considerando a estratégia das CNOPs, observa-se que, assim como LSVs, estas apresentam erros quadráticos menores em 14 dos 20 casos considerados. Nas previsões de 1 dia as CNOPs sempre diminuíram o erro de previsão sobre o ponto $q_{j}=20$ e o erro médio sobre o continente. CNOPs não tiveram um desempenho satisfatório no período de 3 dias, sendo pior que a estratégia aleatória em 5 dos 10 casos. Olhando para as estratégias de CNOPs e LSVs entre si, observa-se que para o ponto $q_{j}=20$, CNOPs sempre foram melhores que LSVs em ambos os períodos de 1 e 3 dias. No entanto sobre o erro médio sobre 


\begin{tabular}{c|c|cc|cc|cc}
\hline \multirow{3}{*}{ Experimento } & \multirow{2}{*}{ Método } & \multicolumn{7}{|c}{ Período de previsão } \\
\cline { 3 - 8 } & & \multicolumn{2}{|c}{1} & \multicolumn{2}{c}{3} & \multicolumn{2}{c}{6} \\
\cline { 3 - 8 } A & RAND & 7.371832 & 3.774349 & 9.813468 & 8.291520 & 7.609477 & 7.369140 \\
& LSV & 7.911623 & 3.281057 & 9.391240 & 8.155663 & 7.487562 & 7.473620 \\
& CNOP & 5.859065 & 3.196150 & 9.567092 & 8.271504 & 7.703841 & 7.594791 \\
\hline \multirow{3}{*}{$\mathrm{B}$} & RAND & 7.013381 & 3.863553 & 9.103662 & 8.361142 & 7.383858 & 7.383858 \\
& LSV & 6.232731 & 2.941640 & 9.701326 & 8.312908 & 7.519055 & 7.519055 \\
& CNOP & 6.264740 & 3.092038 & 10.515728 & 8.395699 & 7.631166 & 7.631166 \\
\hline \multirow{3}{*}{$\mathrm{C}$} & RAND & 8.166559 & 3.697234 & 9.530516 & 8.211614 & 7.673646 & 7.531734 \\
& LSV & 7.777344 & 3.612695 & 8.763646 & 8.188766 & 8.089726 & 7.513160 \\
& CNOP & 6.736503 & 3.534809 & 9.801821 & 8.280502 & 7.824267 & 7.436944 \\
\hline \multirow{3}{*}{$\mathrm{D}$} & RAND & 6.941264 & 3.687297 & 9.632486 & 8.305390 & 7.526964 & 7.395431 \\
& LSV & 7.548874 & 3.269240 & 10.258824 & 8.276984 & 7.541900 & 7.542733 \\
& CNOP & 6.225736 & 3.114122 & 9.826816 & 8.275939 & 7.763763 & 7.537463 \\
\hline \multirow{2}{*}{ E } & RAND & 7.340104 & 3.944720 & 8.983617 & 8.245193 & 7.519504 & 7.350030 \\
& LSV & 7.548874 & 3.269240 & 10.258824 & 8.276984 & 7.541900 & 7.542733 \\
& CNOP & 6.225736 & 3.114122 & 9.826816 & 8.275939 & 7.763763 & 7.537463 \\
\hline
\end{tabular}

Tabela 5.6: Experimentos numéricos com o sistema (5.4) para 5 períodos de 90 dias utilizando CNOPs, LSVs e sorteio para escolher pontos sensiveis nos períodos de 1, 3 e 6 dias.

o continente CNOPs são piores em todos os casos.

A Tabela 5.6 mostra resultados para o período de 90 dias. Se compararmos os erros quadráticos obtidos com a estratégia que usa LSVs e a estratégia aleatória, a estratégia aleatória apresenta erros quadráticos menores em 16 dos 30 experimentos considerados, mostrando que, neste experimento, a utilização de LSVs não ajuda a reduzir o erro de previsão. Observa-se que LSVs apresentam melhores resultados em todos os 10 casos diminuindo o erro médio sobre o continente nas previsões de 1 dia, em apenas 1 caso (do total de 10) para as previsões de 3 dias e em 4 (de um total de 10 casos) para previsões de 6 dias. Nas previsões para o ponto $q_{j}=20$, LSVs não são melhores que a estratégia aleatória em 11 dos 15 casos considerados. Resultados similares podem er observados ao comparar a estratégia aleatória com a estratégia baseada em CNOPs. Em 16 dos 30 casos a estratégia das CNOPs não foi melhor que a escolha aleatória de pontos indicando que, ao menos neste experimento, LSVs e CNOPs não se mostraram alternativas para reduzir o erro de previsão. No entanto, CNOPs foram melhores em todos os experimentos para 1 dia de previsão tanto no ponto $q_{j}=20$ quanto na média sobre o continente. 


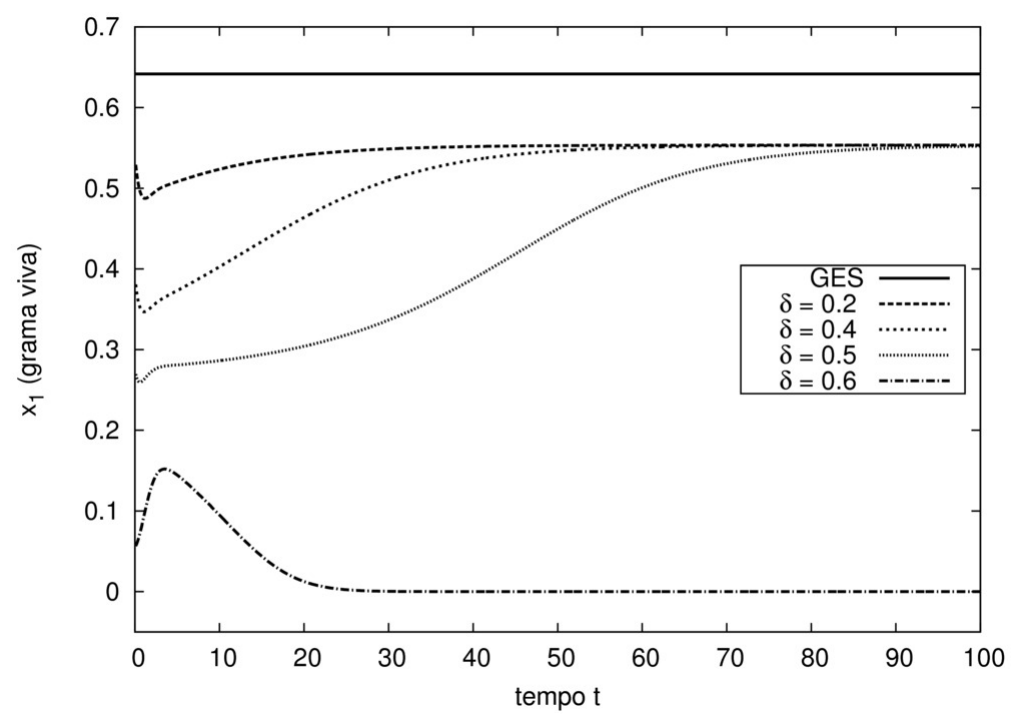

(a) Evolução temporal de $x_{1}$.

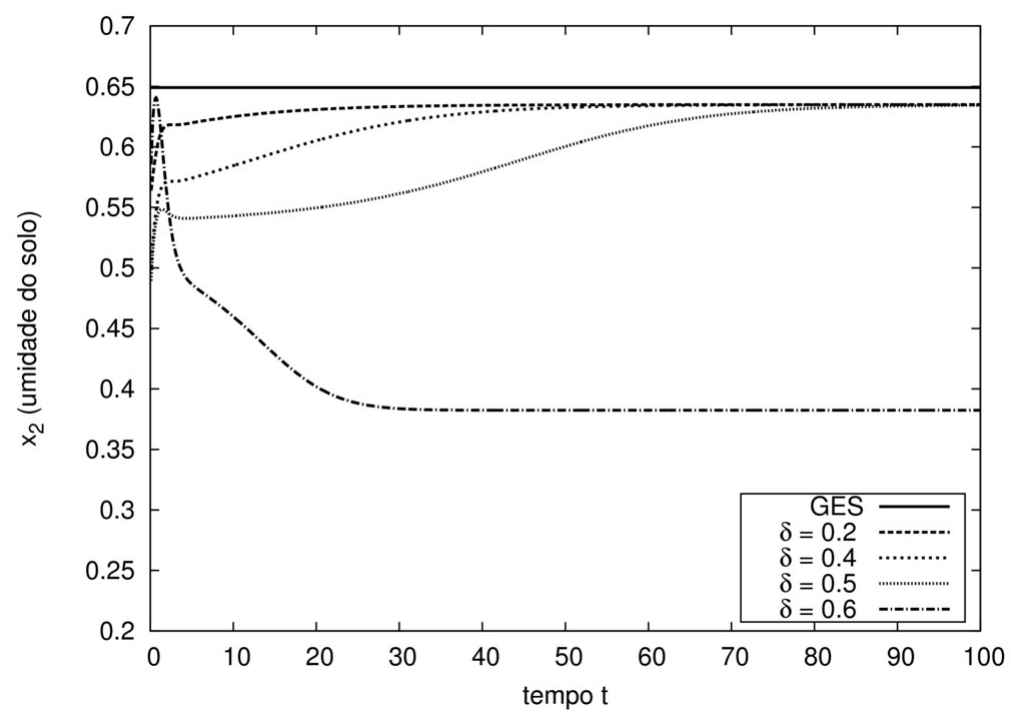

(b) Evolução temporal de $x_{2}$.

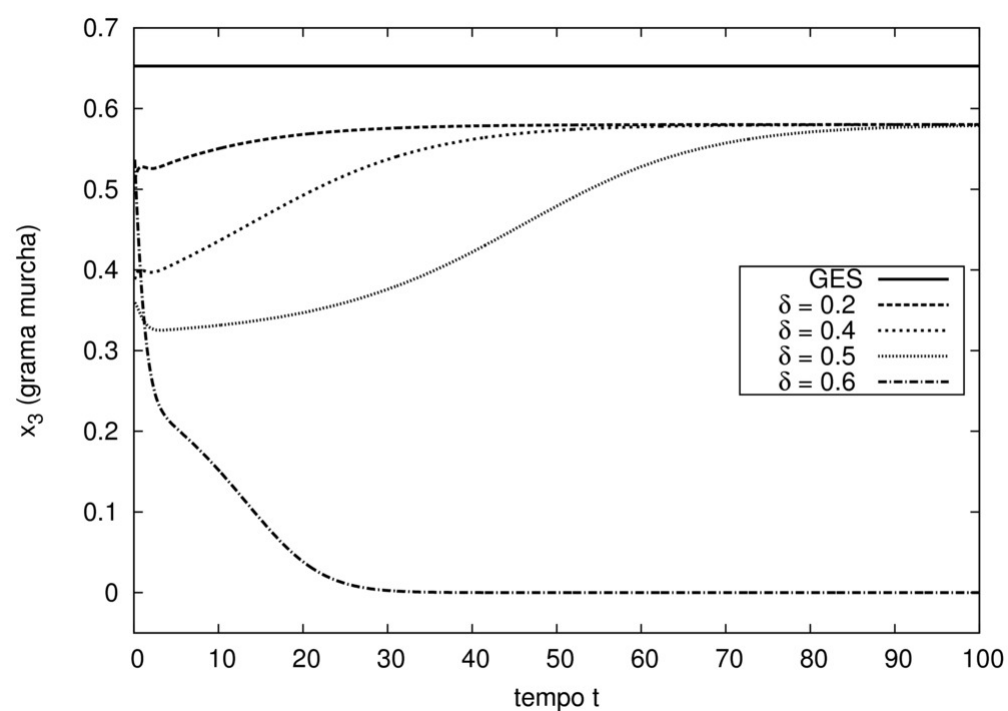

(c) Evolução temporal de $x_{3}$.

Figura 5.4: Evolução no tempo das coordenadas do sistema (5.2). Para cada coordenada são apresentados o sistema em equilibrio GES e o equilibrio perturbado com a CNOP calculada para $\delta=0.2,0.4,0.5$ e 0.6. 


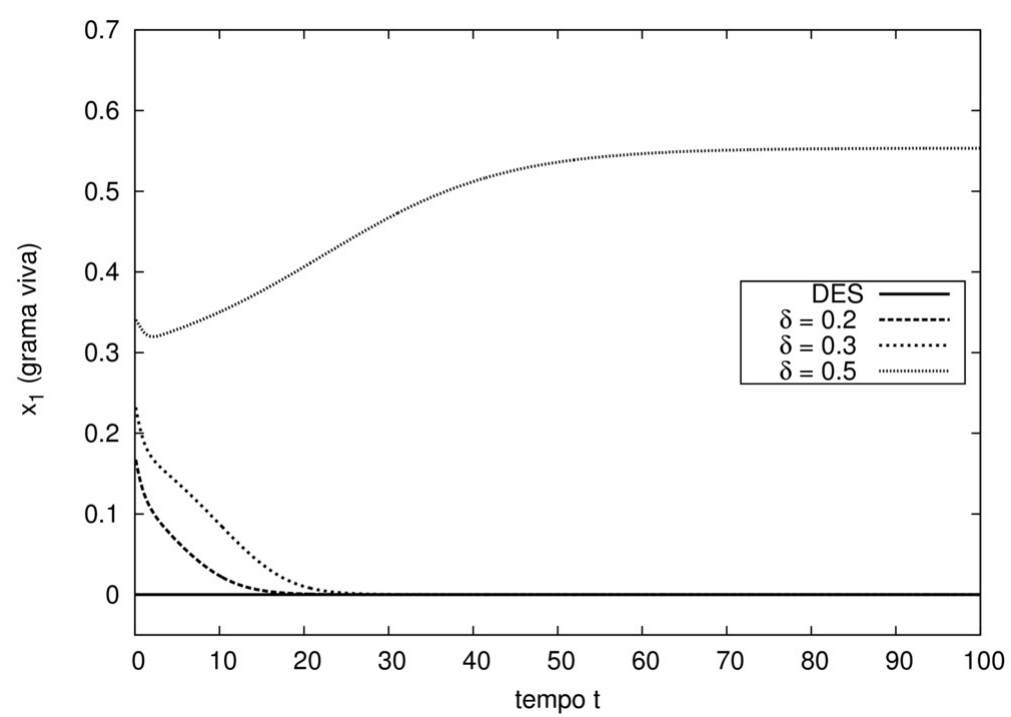

(a) Evolução temporal de $x_{1}$.

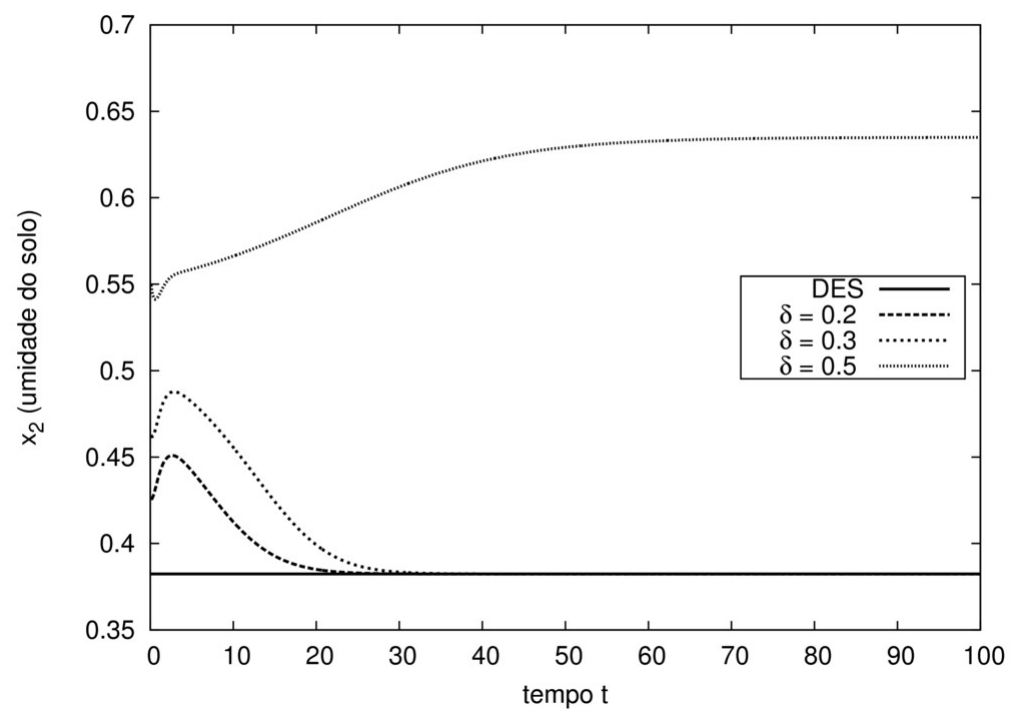

(b) Evolução temporal de $x_{2}$.

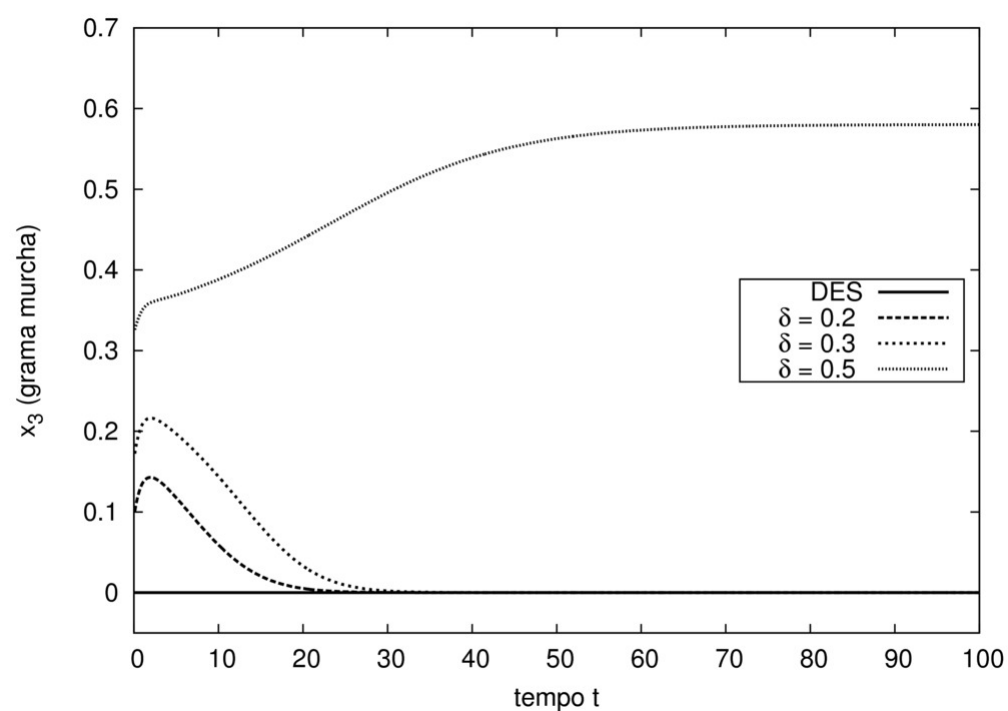

(c) Evolução temporal de $x_{3}$.

Figura 5.5: $O$ mesmo que em 5.4, mas para o sistema em equilibrio DES. As perturbações foram obtidas para $\delta=0.2,0.3$ e 0.5 . 


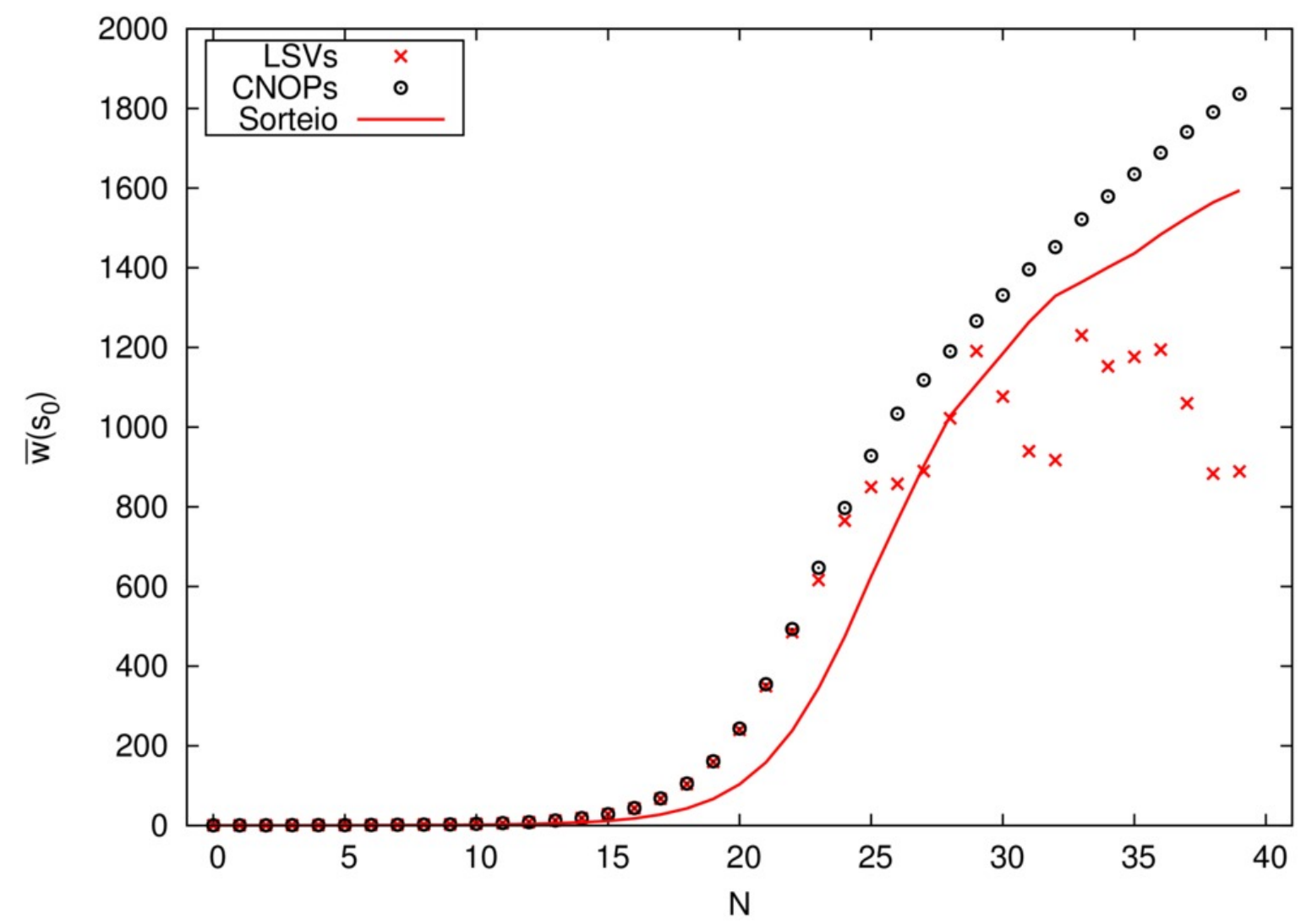

Figura 5.6: Comparação entre resultados de CNOPs, LSVs e escolha aleatória de pontos variando o número de passos de integração no intervalo de $1 \Delta t, \ldots, N \Delta t$, com $N=40$. 


\section{Capítulo 6}

\section{Conclusões}

Neste trabalho analisamos vinte trabalhos na área de Meteorologia que utilizam o método do Gradiente Espectral Projetado (SPG). Constatamos que todos eles utilizam o SPG para o cálculo das chamadas perturbações condicionais não-lineares ótimas (CNOPs). As CNOPs tem aplicações no estudo de estabilidade, sensibilidade e previsibilidade de sistemas meteorológicos modelados como sistemas de equações diferenciais. Estudamos portanto as CNOPs e uma técnica anterior baseada no modelo tangente linear e o calculo de valores e vetores singulares.

Reproduzindo experimentos da literatura constatamos que ambas técnicas dão resultados similares para períodos curtos de integração, ao passo que as CNOPs, ao capturarem de forma adequada a não-linearidade dos modelos, fornecem resultados mais apurados para períodos longos de integração.

O cálculo das CNOPs requer a solução de um problema de otimização contínuo e diferenciável sujeito a um conjunto convexo de restrições. A função objetivo do problema é composta pela integração numérica da equação diferencial, enquanto que as restrições do problema estão relacionadas a restrições físicas na perturbação da condição inicial do processo de integração. Utilizamos RungeKutta de ordem 4 como método de integração e, baseados nas formulas de FAD (Fast Automatic Differentiation) introduzidas no contexto de problemas de controle ótimo, calculamos os gradiente exato da função objetivo. Desenvolvemos também técnicas simples baseadas em multistart para aumentar as chances de encontrar a solução global dos problemas de otimização. Com esse ferramental, ilustramos o uso das CNOPs em três problemas diferentes para lidar com os problemas de estabilidade, sensibilidade e previsibilidade.

Com o trabalho acima descrito, cumprimos com nosso objetivo inicial de compreender o uso do SPG no contexto de problemas de Meteorologia. 


\section{Apêndice A}

\section{Código fonte dos modelos}

O código fonte dos modelos estudados neste trabalho encontram-se listados abaixo.

\section{A.1 O Modelo de Lorenz de 1963}

Abaixo, rotinas em ANSI C que implementam, respectivamente, o sistema de Lorenz de 1963 e o cálculo do seu jacobiano. Maiores detalhes sobre o modelo podem ser encontrados na Seção 5.1.

A rotina lorenz63 recebe como entrada a dimensão do sistema em neqs e os vetores $\mathrm{x}[]$ e y[] de dimensão neqs. Devolve os valores da função em y[].

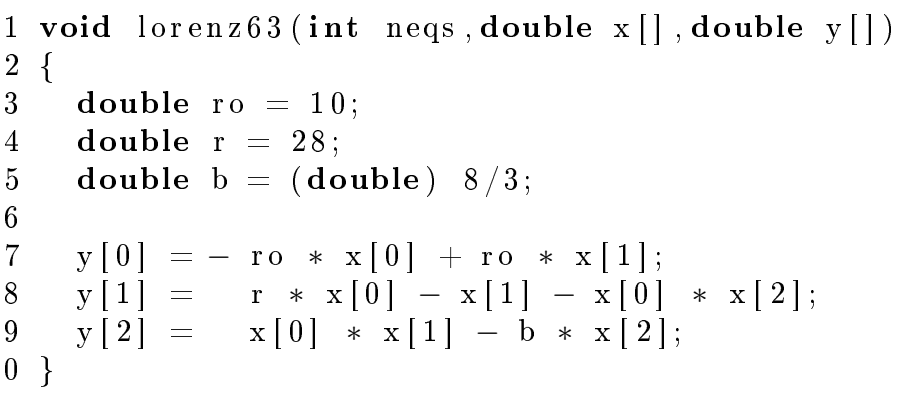

A rotina lorenz63D implementa o Jacobiano do sistema de Lorenz de 1963. Recebe como entrada a dimensão do sistema em neqs, um vetor x[] de dimensão neqs, e a matriz dydx[П com dimensão neqs $\mathrm{x}$ neqs. Devolve o Jacobiano do sistema na matriz dydx[[]].

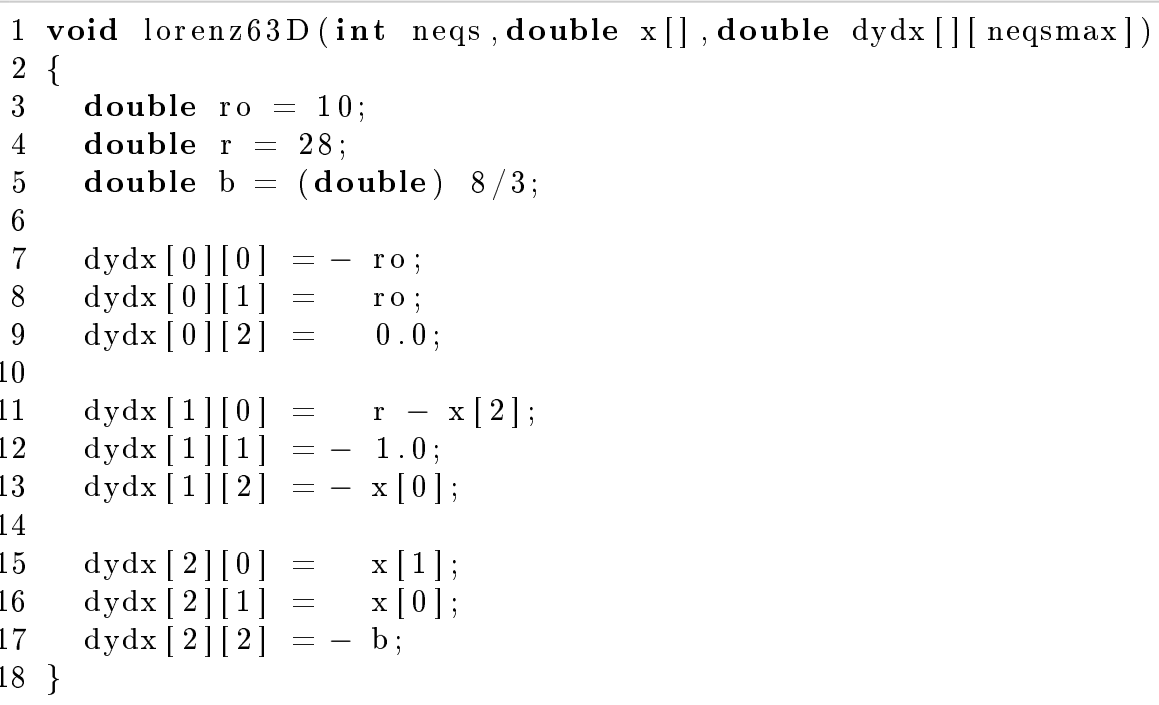




\section{A.2 O Modelo simplificado de ecossistema}

Rotinas em ANSI C que implementam o modelo de ecossistema simplificado e o seu jacobiano estudados na Seção 5.2.

A rotina ecomodel recebe como entrada a dimensão do sistema em neqs e os vetores $\mathrm{x} \|$ e $\mathrm{y} \| \mathrm{de}$ dimensão neqs. Devolve os valores da função em y[].

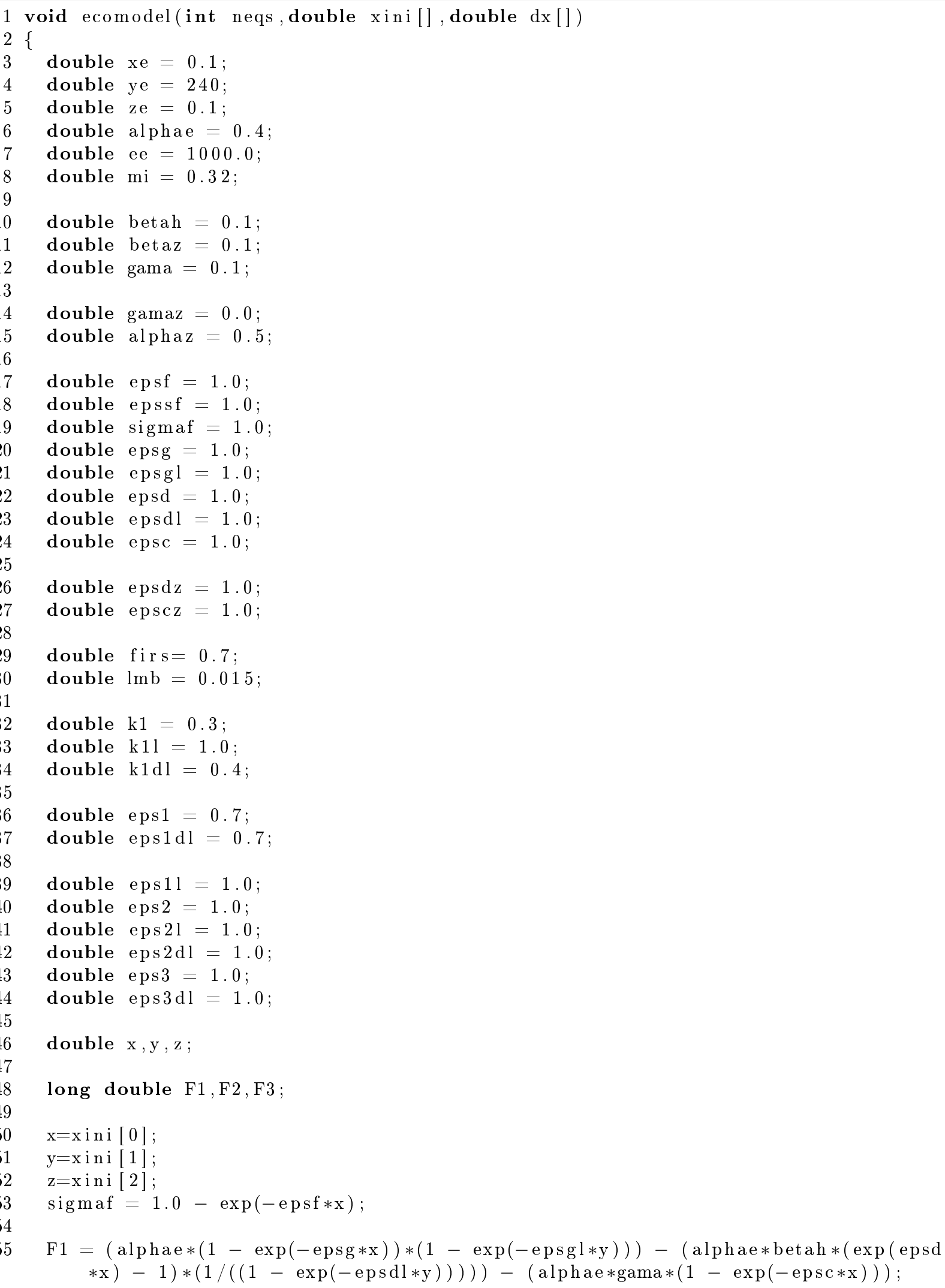


56

61

62

63

64

$65\}$

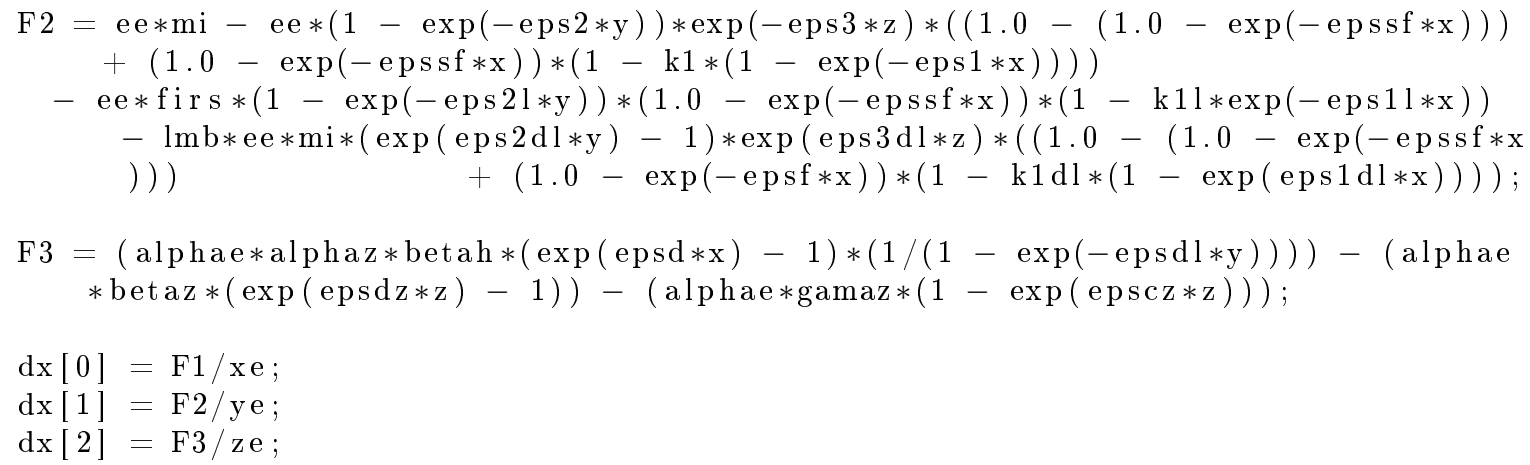

A rotina ecomodelD implementa o Jacobiano do sistema de modelo de ecossistema simplificado. Recebe como entrada a dimensão do sistema em neqs, um vetor x[] de dimensão neqs, e a matriz dydx [П com dimensão neqs x neqs. Devolve o Jacobiano do sistema na matriz dydx [[].

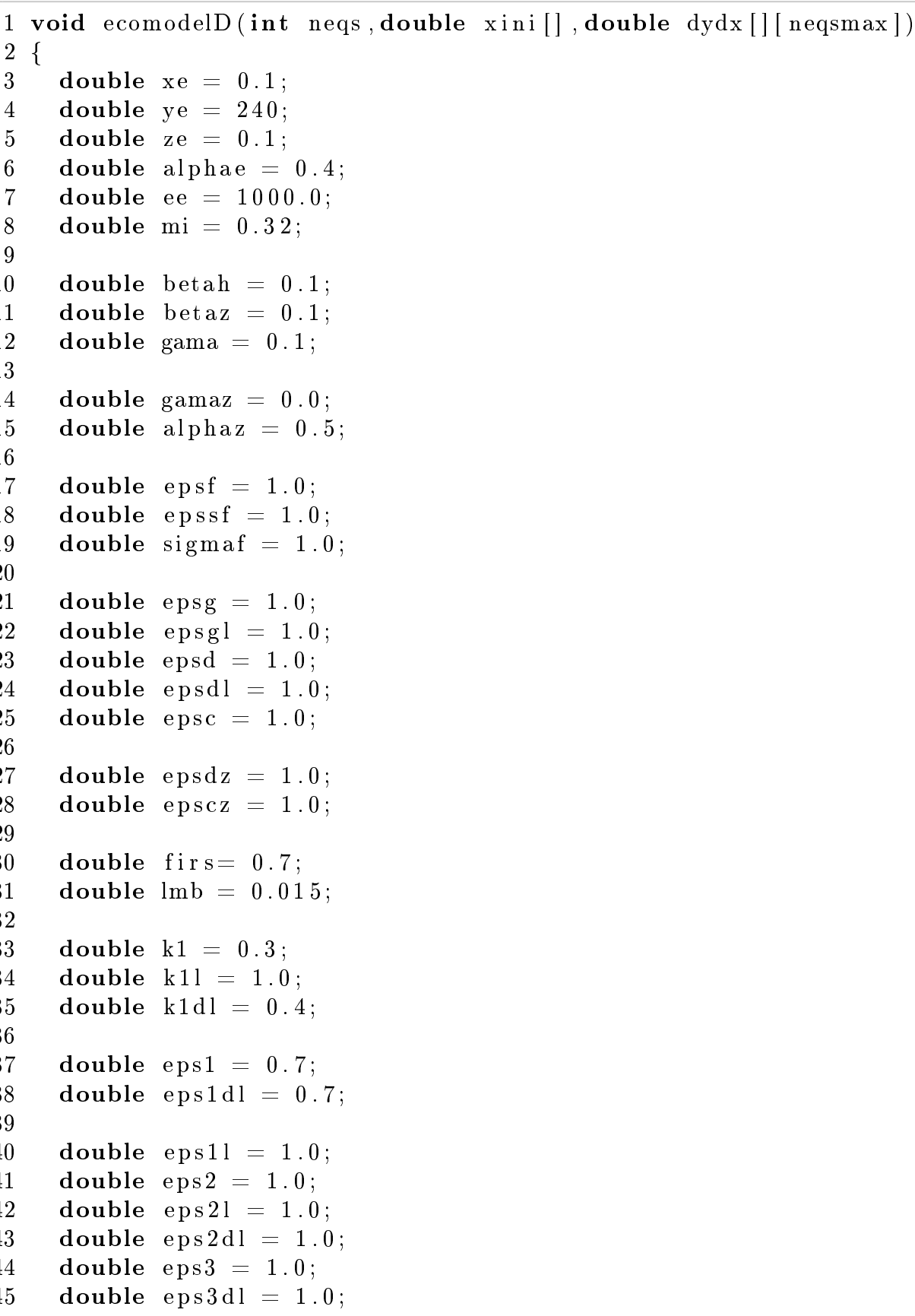


46

47

48

49

50

51

52

53

54

55

double $\mathrm{x}, \mathrm{y}, \mathrm{z}$;

double $\mathrm{dF} 1 \mathrm{dx}, \mathrm{dF} 1 \mathrm{dy}, \mathrm{dF} 1 \mathrm{dz}, \mathrm{dF} 2 \mathrm{dx}, \mathrm{dF} 2 \mathrm{dy}, \mathrm{dF} 2 \mathrm{dz}, \mathrm{dF} 3 \mathrm{dx}, \mathrm{dF} 3 \mathrm{dy}, \mathrm{dF} 3 \mathrm{dz}$;

$\mathrm{x}=\mathrm{xini}[0]$;

$\mathrm{y}=\mathrm{xini}[1]$

$\mathrm{z}=\mathrm{xini}[2]$;

$\operatorname{sigmaf}=1.0-\exp (-\operatorname{epsf} * \mathrm{x})$;

$\mathrm{dF} 1 \mathrm{dx}=-$ alphae $*$ betah $* \operatorname{eps} d * \exp (\operatorname{epsd} * \mathrm{x}) /(1-\exp (-\operatorname{eps} \mathrm{d} l * \mathrm{y}))-\operatorname{alphae} * \operatorname{epsc} * \operatorname{gama} *$ $\exp (-\operatorname{epsc} * \mathrm{x})+\operatorname{alphae} * \operatorname{epsg} *(1-\exp (-\operatorname{epsgl} * \mathrm{y})) * \exp (-\operatorname{epsg} * \mathrm{x}) ;$

$\mathrm{dF} 2 \mathrm{dx}=-\mathrm{ee} * \operatorname{eps} 1 \mathrm{l} * \mathrm{firs} * \mathrm{k} 1 \mathrm{l} *(1-\exp (-\operatorname{eps} 2 \mathrm{l} * \mathrm{y})) *(1.0-\exp (-\operatorname{epssf} * \mathrm{x})) * \exp (-$ $\operatorname{eps} 11 * x)-\operatorname{ee} * \operatorname{epssf} * \mathrm{firs} *(1-\exp (-\operatorname{eps} 21 * \mathrm{y})) *(-\mathrm{k} 11 * \exp (-\operatorname{eps} 11 * \mathrm{x})+1) * \exp (-$ $\operatorname{epssf} * \mathrm{x})-\operatorname{ee} * \operatorname{lmb} * \mathrm{mi} *(\exp (\operatorname{eps} 2 \mathrm{dl} * \mathrm{y})-1) *(\operatorname{eps} 1 \mathrm{dl} * \mathrm{k} 1 \mathrm{dl} *(1.0-\exp (-\operatorname{epsf} * \mathrm{x})) *$ $\exp (\operatorname{eps} 1 \mathrm{dl} * \mathrm{x})+\operatorname{epsf} *(-\mathrm{k} 1 \mathrm{dl} *(-\exp (\operatorname{eps} 1 \mathrm{dl} * \mathrm{x})+1)+1) * \exp (-\operatorname{epsf} * \mathrm{x})-\operatorname{epssf} *$ $\exp (-\operatorname{epss} f * x)) * \exp (\operatorname{eps} 3 \mathrm{~d} l * z)-\operatorname{ee} *(1-\exp (-\operatorname{eps} 2 * \mathrm{y})) *(-\operatorname{eps} 1 * \mathrm{k} 1 *(1.0-\exp (-$ $\operatorname{epssf} * \mathrm{x})) * \exp (-\operatorname{eps} 1 * \mathrm{x})+\operatorname{epssf} *(-\mathrm{k} 1 *(1-\exp (-\operatorname{eps} 1 * \mathrm{x}))+1) * \exp (-\operatorname{epssf} * \mathrm{x})-$ $\operatorname{epssf} * \exp (-\operatorname{epssf} * \mathrm{x})) * \exp (-\operatorname{eps} 3 * \mathrm{z})$

$\mathrm{dF} 3 \mathrm{dx}=\operatorname{alphae} * \operatorname{alphaz} * \operatorname{betah} * \operatorname{epsd} * \exp (\operatorname{epsd} * \mathrm{x}) /(1-\exp (-\operatorname{epsdl} * \mathrm{y}))$;

$\mathrm{dF} 1 \mathrm{dy}=\operatorname{alphae} * \operatorname{betah} * \operatorname{epsdl} *(\exp (\operatorname{epsd} * \mathrm{x})-1) * \exp (-\operatorname{epsdl} * \mathrm{y}) /$ pow $((1-\exp (-\operatorname{epsdl}$ $* \mathrm{y})), 2)+\operatorname{alphae} * \operatorname{epsgl} *(1-\exp (-\operatorname{epsg} * \mathrm{x})) * \exp (-\operatorname{epsgl} * \mathrm{y})$;

$\mathrm{dF} 2 \mathrm{dy}=-\mathrm{ee} * \operatorname{eps} 2 *((1.0-\exp (-\operatorname{epssf} * \mathrm{x})) *(-\mathrm{k} 1 *(1-\exp (-\operatorname{eps} 1 * \mathrm{x}))+1)+\exp (-$ $\operatorname{epssf} * \mathrm{x})) * \exp (-\operatorname{eps} 2 * \mathrm{y}) * \exp (-\operatorname{eps} 3 * \mathrm{z})-\operatorname{ee} * \operatorname{eps} 2 \mathrm{dl} * \operatorname{lmb} * \operatorname{mi} *((1.0-\exp (-\operatorname{epsf} * \mathrm{x})$ )$*(-\mathrm{k} 1 \mathrm{dl} *(-\exp (\operatorname{eps} 1 \mathrm{~d} l * \mathrm{x})+1)+1)+\exp (-\operatorname{epssf} * \mathrm{x})) * \exp (\operatorname{eps} 2 \mathrm{~d} l * \mathrm{y}) * \exp ($ $\operatorname{eps} 3 \mathrm{dl} * \mathrm{z})-\operatorname{ee} * \operatorname{eps} 21 * \mathrm{firs} *(1.0-\exp (-\operatorname{epssf} * \mathrm{x})) *(-\mathrm{k} 1 \mathrm{l} * \exp (-\operatorname{eps} 11 * \mathrm{x})+1) *$ $\exp (-\operatorname{eps} 21 * y)$;

$\mathrm{dF} 3 \mathrm{dy}=-\operatorname{alphae} * \operatorname{alphaz} * \operatorname{betah} * \operatorname{epsdl} *(\exp (\operatorname{epsd} * \mathrm{x})-1) * \exp (-\operatorname{epsdl} * \mathrm{y}) / \operatorname{pow}((1-$ $\exp (-\operatorname{epsdl} * \mathrm{y})), 2)$;

$\mathrm{dF} 1 \mathrm{dz}=0$

$\mathrm{dF} 2 \mathrm{dz}=\operatorname{ee} * \operatorname{eps} 3 *(1-\exp (-\operatorname{eps} 2 * \mathrm{y})) *((1.0-\exp (-\operatorname{epssf} * \mathrm{x})) *(-\mathrm{k} 1 *(1-\exp (-\operatorname{eps} 1 *$

$\mathrm{x}))+1)+\exp (-\operatorname{epssf} * \mathrm{x})) * \exp (-\operatorname{eps} 3 * \mathrm{z})-\operatorname{ee} * \operatorname{eps} 3 \mathrm{dl} * \operatorname{lmb} * \operatorname{mi} *((1.0-\exp (-\operatorname{epsf}$

$* \mathrm{x})) *(-\mathrm{k} 1 \mathrm{dl} *(-\exp (\operatorname{eps} 1 \mathrm{dl} * \mathrm{x})+1)+1)+\exp (-\operatorname{epssf} * \mathrm{x})) *(\exp (\operatorname{eps} 2 \mathrm{dl} * \mathrm{y})-1) *$ $\exp (\operatorname{eps} 3 \mathrm{dl} * \mathrm{z})$

$\mathrm{dF} 3 \mathrm{dz}=-\operatorname{alphae} * \operatorname{betaz} * \operatorname{epsdz} * \exp (\operatorname{epsdz} * z)+\operatorname{alphae} * \operatorname{epscz} * \operatorname{gamaz} * \exp (\operatorname{epscz} * z) ;$

$\operatorname{dydx}[0][0]=\mathrm{dF} 1 \mathrm{dx} / \mathrm{xe}$;

$\operatorname{dydx}[0][1]=\mathrm{dF} 1 \mathrm{dy} / \mathrm{xe}$;

$\operatorname{dydx}[0][2]=\mathrm{dF} 1 \mathrm{dz} / \mathrm{xe}$;

$\operatorname{dydx}[1][0]=\mathrm{dF} 2 \mathrm{dx} / \mathrm{ye}$;

$\operatorname{dydx}[1][1]=\mathrm{dF} 2 \mathrm{dy} / \mathrm{ye}$;

$\operatorname{dydx}[1][2]=\mathrm{dF} 2 \mathrm{dz} / \mathrm{ye}$;

$\operatorname{dydx}[2][0]=\mathrm{dF} 3 \mathrm{dx} / \mathrm{ze}$;

$\operatorname{dydx}[2][1]=\mathrm{dF} 3 \mathrm{dy} / \mathrm{ze}$;

$\operatorname{dydx}[2][2]=\mathrm{dF} 3 \mathrm{dz} / \mathrm{ze}$; 


\section{A.3 O Modelo de Lorenz de 1996}

Rotinas utilizadas nos estudos realizados conforme o descrito na Seção 5.3.

A rotina lorenz96 recebe como entrada a dimensão do sistema em neqs e os vetores $\mathrm{x}[$ e $\mathrm{y}[] \mathrm{de}$ dimensão neqs. Devolve os valores da função em y[].

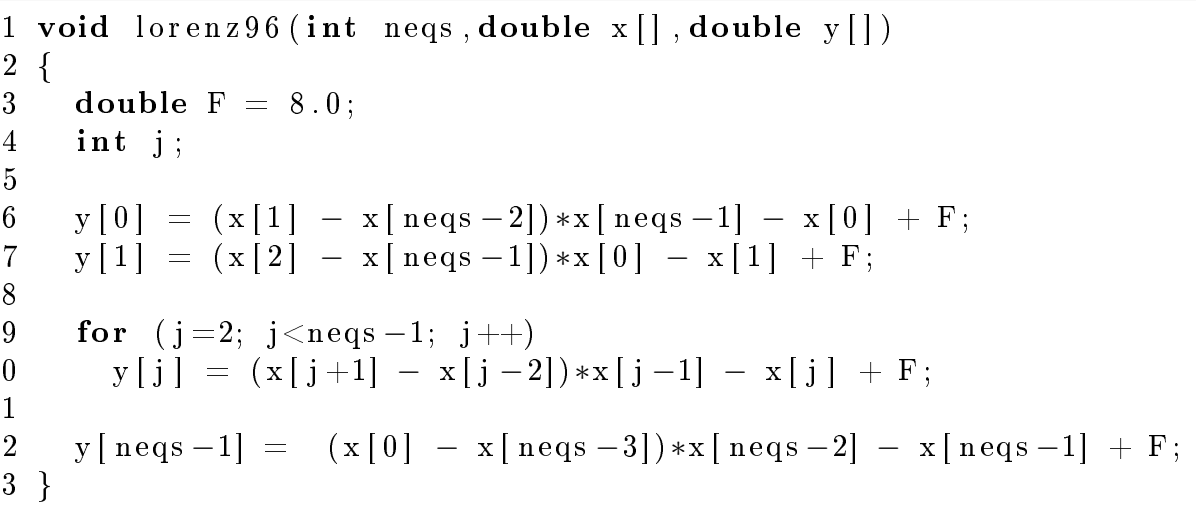

A rotina lorenz96D implementa o Jacobiano do sistema de Lorenz de 1996. Recebe como entrada a dimensão do sistema em neqs, um vetor $\mathrm{x}[]$ de dimensão neqs, e a matriz dydx[П com dimensão neqs $\mathrm{x}$ neqs. Devolve o Jacobiano do sistema na matriz dydx[][].

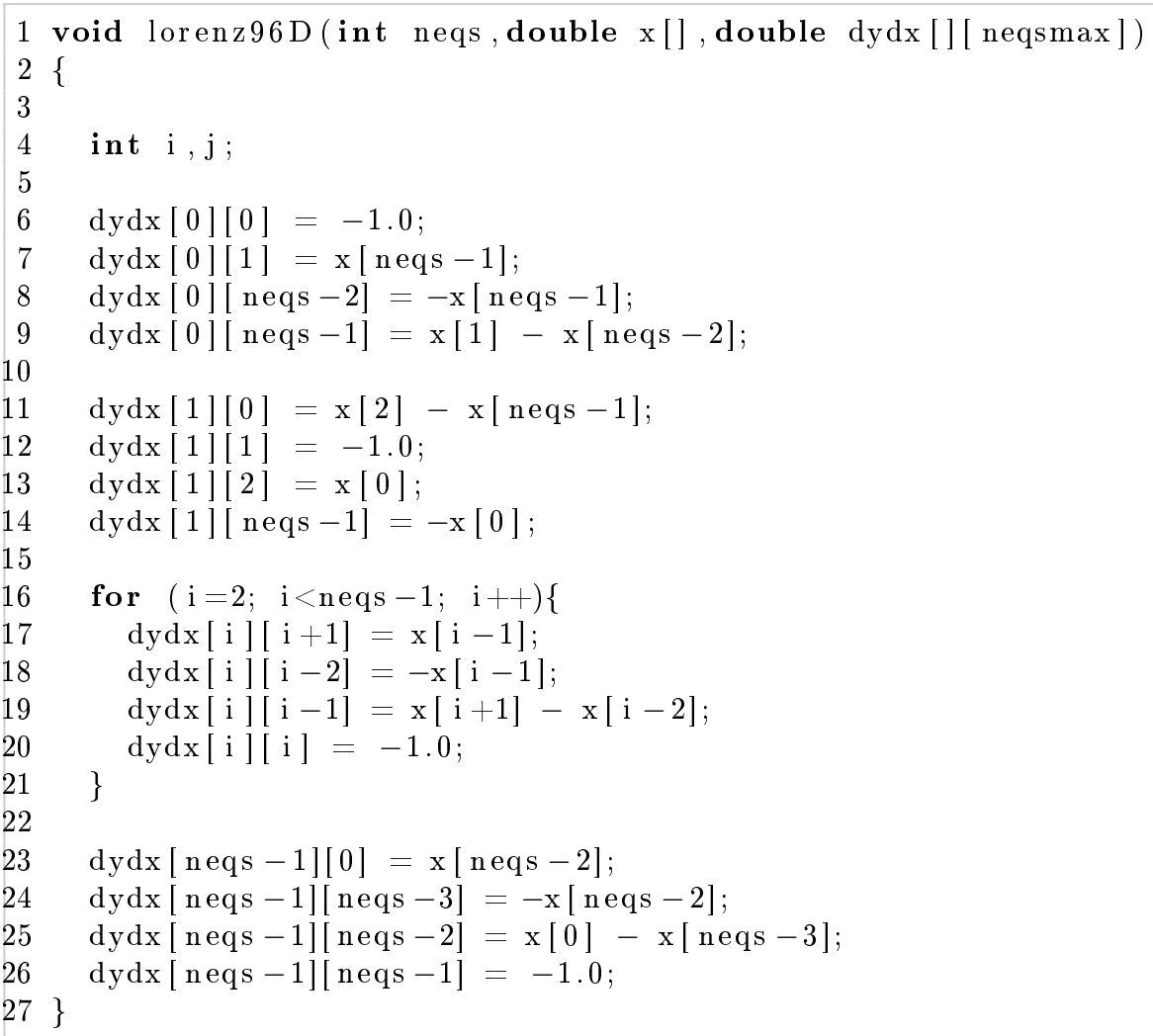




\section{Referências Bibliográficas}

[1] E.G. Birgin, J.M. Martínez, and M. Raydan. Algorithm 813: SPG software for convexconstrained optimization. ACM Transactions on Mathematical Software (TOMS), 27(3):340349, 2001. 1

[2] E.G. Birgin, J.M. Martínez, and D.P. Ronconi. Optimizing the packing of cylinders into a rectangular container: a nonlinear approach. European Journal of Operational Research, 160(1):19-33, 2005. 1

[3] W.S. Duan and M. Mu. Conditional nonlinear optimal perturbation: Applications to stability, sensitivity, and predictability. Science in China Series D: Earth Sciences, 52(7):883-906, 2009. $1,2,9$

[4] M. Mu, F. Zhou, and H. Wang. A method for identifying the sensitive areas in targeted observations for tropical cyclone prediction: Conditional nonlinear optimal perturbation. Monthly Weather Review, 137(5):1623-1639, 2009. 1, 2

[5] Z.N. Jiang, M. Mu, and D.H. Wang. Ensemble prediction experiments using conditional nonlinear optimal perturbation. Science in China Series D: Earth Sciences, 52(4):511-518, 2009. 1,2

[6] J. Wang and J. Li. A four-dimensional scheme based on singular value decomposition (4DSVD) for chaotic-attractor-theory-oriented data assimilation. Journal of Geophysical Research, 114:D2, 2009. 1, 2

[7] Z. Jiang, M. Mu, and D. Wang. Conditional nonlinear optimal perturbation of a T21L3 quasigeostrophic model. Quarterly Journal of the Royal Meteorological Society, 134(633):1027-1038, 2008. 1,2

[8] M. Mu, H. Xu, and WS Duan. A kind of initial errors related to spring predictability barrier for El Nino events in Zebiak-Cane model. Geophysical Research Letters, 34:L03709, 2007. 1, 2

[9] X. Hui, D. Wansuo, and W. Jianchao. The Tangent Linear Model and Adjoint of a Coupled Ocean-Atmosphere Model and Its Application to the Predictability of ENSO. In Geoscience and Remote Sensing Symposium, 2006. IGARSS 2006. IEEE International Conference on, pages 640-643. IEEE, 2007. 1, 2

[10] Z. Jiang. Applications of conditional nonlinear optimal perturbation to the study of the stability and sensitivity of the Jovian atmosphere. Advances in Atmospheric Sciences, 23(5):775-783, 2006. 1,2

[11] H. Xu and W. Duan. What kind of initial errors cause the severest prediction uncertainty of El Niño in Zebiak-Cane model. Advances in Atmospheric Sciences, 25(4):577-584, 2008. 1, 2

[12] W. Duan, H. Xu, and M. Mu. Decisive role of nonlinear temperature advection in El Nino and La Nina amplitude asymmetry. Journal of Geophysical Research, 113(C1):C01014, 2008. 1, 2 
[13] Z. Jiang and D. Wang. A study on precursors to blocking anomalies in climatological flows by using conditional nonlinear optimal perturbations. Quarterly Journal of the Royal Meteorological Society, 136(650):1170-1180, 2010. 1, 2

[14] M. Mu and Z. Zhang. Conditional nonlinear optimal perturbations of a two-dimensional quasigeostrophic model. Journal of the Atmospheric Sciences, 63(6):1587-1604, 2006. 1, 2

[15] D. Wan-suo and M. Mu. Applications of nonlinear optimization method to numerical studies of atmospheric and oceanic sciences. Applied Mathematics and Mechanics, 26(5):636-646, 2005. 1,2

[16] E.N. Lorenz. A study of the predictability of a 28-variable atmospheric model. Tellus, $17(3): 321-333,1965.1$

[17] M. Mu. Nonlinear singular vectors and nonlinear singular values. Science in China Series D: Earth Sciences, 43(4):375-385, 2000. 2

[18] M. Mu and W. Duan. A new approach to studying ENSO predictability: Conditional nonlinear optimal perturbation. Chinese Science Bulletin, 48(10):1045-1047, 2003. 2

[19] M. Mu and B. Wang. Nonlinear instability and sensitivity of a theoretical grassland ecosystem to finite-amplitude perturbations. Nonlinear Processes in Geophysics, 14(4):409-423, 2007. 2, $25,27,28$

[20] A.H. Nayfeh and B. Balachandran. Applied nonlinear dynamics, volume 2. Wiley Online Library, 1995. 3, 6

[21] S.L. Campbell and R. Haberman. Introduction to Differential Equations with Dynamical Systems. Princeton University Press, 2008. 3, 14

[22] G.H. Golub and C.F. Van Loan. Matrix computations. Johns Hopkins University Press, 1996. 5

[23] J.M. Lewis, S. Lakshmivarahan, and S.K. Dhall. Dynamic data assimilation: a least squares approach, volume 104. Cambridge Univ Pr, 2006. 7

[24] J.H. Mathews. Numerical methods for mathematics, science, and engineering. Prentice Hall International Editions, 1992. 7

[25] E. Kalnay. Atmospheric modeling, data assimilation, and predictability. Cambridge University Press, 2003. 7

[26] E.G. Birgin and Y.G. Evtushenko. Automatic differentiation and spectral projected gradient methods for optimal control problems. Optimization Methods and Software, 10(2):125-146, 1998. 9,11

[27] E.G. Birgin, R. Marcos, et al. Spg: Software for convex-constrained optimization. ACM Transactions on Mathematical Software, 27:340-349, 2001. 10

[28] E.G. Birgin, J.M. Martínez, and M. Raydan. Nonmonotone spectral projected gradient methods on convex sets. SIAM Journal on Optimization, 10(4):1196-1211, 2000. 10

[29] E.N. Lorenz. Deterministic nonperiodic flow. Atmos J Sci, 20:130-141, 1963. 12, 21

[30] T.N. Palmer. Extended-range atmospheric prediction and the Lorenz model. Bulletin of the American Meteorological Society, 74(1), 1993. 12, 13, 21

[31] T.N. Palmer. Predicting uncertainty in forecasts of weather and climate. Reports on Progress in Physics, 63:71, 2000. 12, 21, 23 
[32] H.E. Moolenaar and F.M. Selten. Finding the effective parameter perturbations in atmospheric models: the lorenz63 model as case study. Tellus A, 56(1):47-55, 2004. 12, 21

[33] E. Evans, N. Bhatti, J. Kinney, L. Pann, M. Peña, S.C. Yang, E. Kalnay, and J. Hansen. Rise: Undergraduates find that regime changes in Lorenz's model are predictable. Bulletin-American Meteorological Society, 85(4):520-524, 2004. 12, 13, 21

[34] H. Mukougawa, M. Kimoto, and S. Yoden. A relationship between local error growth and quasi-stationary states: Case study in the Lorenz system. Journal of the Atmospheric Sciences, 48(10):1231-1237, 1991. 12, 21

[35] C. Sparrow. The Lorenz equations: bifurcations, chaos, and strange attractors. Springer-Verlag, 1982. 12,21

[36] M. Galassi et al. Gnu scientific library reference manual. 13

[37] A.A. Zhiglavskiǔ, A. Zhilinskas, and A. Žilinskas. Stochastic global optimization. Springer Verlag, 2008. 13

[38] X. Zeng, S.S.P. Shen, X. Zeng, and R.E. Dickinson. Multiple equilibrium states and the abrupt transitions in a dynamical system of soil water interacting with vegetation. Geophysical Research Letters, 31:5501, 2004. 25

[39] Q.C. Zeng, P.S. Lu, and X.D. Zeng. Maximum simplified dynamic model of grass field ecosystem with two variables. Science in China series a Mathematics Physics Astronomy and Technological Sciences, 37:94-94, 1994. 25

[40] Q. Zeng and X. Zeng. An analytical dynamic model of grass field ecosystem with two variables. Ecological modelling, 85(2-3):187-196, 1996. 25

[41] E.N. Lorenz and K.A. Emanuel. Optimal sites for supplementary weather observations: Simulation with a small model. Journal of the Atmospheric Sciences, 55(3):399-414, 1998. 28, 29

[42] A. Trevisan and F. Uboldi. Assimilation of standard and targeted observations within the unstable subspace of the observation-analysis-forecast cycle system. Journal of the Atmospheric Sciences, 61(1):103-113, 2004. 28

[43] E.N. Lorenz. Predictability: A problem partly solved. In Proc. Seminar on Predictability, volume 1, 1996. 28 